\title{
KASL clinical practice guidelines: management of hepatitis C
}

\author{
The Korean Association for the Study of the Liver (KASL)*
}

Keywords: Hepatitis C; Management; Guidelines; KASL

\section{PREAMBLE}

\begin{abstract}
Aims
The Korean Association for the Study of the Liver (KASL) Practice Guidelines for Management of Hepatitis C were first established in 2004, and revised in 2013, when direct-acting antivirals (DAA) were not approved in Korea. Since then, numerous studies of the efficacy, adverse effects and drug-drug interactions (DDI) of interferon-free DAA combination therapy have been published. With all oral DAA therapy showing a sustained virologic response
\end{abstract}

(SVR) rate of 80-90\% with minimal adverse events, hepatitis C virus (HCV) eradication is a realistic goal of treatment. Therefore, a screening strategy for HCV-infected populations according to each country's epidemiology and disease burden is required.

DAA combination therapeutics were approved and adapted to practice in Korea in 2015, and KASL revised the guidelines based on a systematic approach that reflects evidence-based medicine and expert opinions.

The clinical practice guidelines for the management of hepatitis $C$ have been revised to be useful for treatment, research and education. These recommendations are not absolute standards of

\footnotetext{
Abbreviations:

3TC, lamivudine; AAR, AST/ALT ratio; ABC, abacavir; AGREE, Appraisal of guidelines for research and evaluation; ALT, alanine transaminase; anti-HCV, antibody to hepatitis C; APRI, aminotransferase-platelet ration index; ARFI, acoustic radiation force; AST, aspartate aminotransferase; ATV, atazanavir; /r, /ritonavir;/c,/cobicistat; Cat, catalyzed by human cathepsin; CEA, carboxylesterase; CEVR, complete EVR; CLIA, chemiluminescent immunoassay; $\mathrm{CrCl}$, creatinine clearance; CTP, Child-Pugh Turcotte; DAA, direct-acting antivirals; DDI, drug-drug interactions; DRV, darunavir; DTG, dolutegravir; DVR, delayed virologic response; ECLIA, electrochemiluminescence; EFV, efavirenz; EIA, enzyme immunoassay; ESRD, end-stage renal disease; ETR, end-of treatment response; ETR, etravirine; EVG, elvitegravir; EVR, early virologic response; FIB4, fibrosis-4 score; FPV, fosamprenavir; FTC, emtricitabine; G-CSF, granulocyte colony stimulating factor; GFR, glomerular filtration rate; GRADE, grading of recommendations, assessment, development and evaluation; HAV, hepatitis A virus; HBV, hepatitis B virus; $\mathrm{HCC}$, hepatocellular carcinoma; $\mathrm{HCV}$, hepatitis $\mathrm{C}$ virus; HINT, histidine triad nucleotidebinding protein; HIRA, Health Insurance Review and Assessment Service; HIV, human immunodeficiency virus; IL 28B, interleukin 28B; IVDU, intravenous drug user; KASL, Korean Association for the Study of the Liver; KCDC, Korea Centers for Disease Control and Prevention; KHF, Korea Hemophilia Foundation; LC, liver cirrhosis; LPV, lopinavir; MELD, Model for End-Stage Liver Disease; MR, magnetic resonance; MVC, maraviroc; ddi, didanosine; NNRTIs, non-nucleoside reverse transcriptase inhibitors; NS, nonstructural; NVP, nevirapine; OATP, organic anion-transporting polypeptide; Opr+D, ombitasvir/paritaprevir/ritonavir and dasabuvir; PCR, polymerase chain reaction; PegIFN, peginterferon; pEVR, partial EVR; PI, protease inhibitor; PR, pegylated interferona+ribavirin therapy; PWID, people who inject drugs; $R^{*}$, ribavirin started from $600 \mathrm{mg} /$ d; R, weight-based ribavirin; RAL, raltegravir; RAV, resistance-associated variants; RFMP, restriction fragment mass polymorphism; RIBA, recombinant immunoblot assays; $R P V$, rilpivirine; RVR, rapid virologic response; $S N P$, single nucleotide polymorphism; SQV, saquinavir; SVR, sustained virologic response; TDF, tenofovirdisoproxil fumarate; TMA, transcription-mediated amplification; TPV, tipranavir; TSH, Thyroid-stimulating hormone; UTR, untranslated region; ZDV, zidovudine
}

Corresponding author : KASL (Committee Chair: Sook-Hyang Jeong) Room A1210 MapoTrapalace, 53 Mapo-daero, Mapo-gu, Seoul 04158, Korea Tel: +82-2-703-0051, Fax: +82-2-703-0071

E-mail: kasl@kams.or.kr

\section{* Clinical Practice Guidelines Committee of KASL for the Management of Hepatitis C}

Sook-Hyang Jeong (Committee Chair, Seoul National University College of Medicine), Jung II Lee (Yonsei University College of Medicine), Young Seok Kim (SoonChunHyang University Bucheon Hospital), Kyung-Ah Kim (Inje Unversity Ilsan Paik Hospital), Sang Hoon Park (Hallym University Kangnam Sacred Heart Hospital), Byung Seok Lee (Chungnam National University College of Medicine), Jun Yong Park (Yonsei University College of Medicine), Ki Tae Yoon (Pusan National University School of Medicine), Young Kul Jung (Korea University College of Medicine), Chang Wook Kim (Catholic University College of Medicine), Eun Young Cho (Wonkwang University School of Medicine), Geum-Youn Gwak (Sungkyunkwan University School of Medicine), Woo Jin Chung (Keimyung University School of Medicine), Jeong Han Kim (Konkuk University School of Medicine)

Received : Feb. 15, 2016/ Accepted : Feb. 22, 2016 
The Korean Association for the Study of the Liver (KASL) KASL clinical practice guidelines: Management of Hepatitis C

care, and adoption of the guidelines in clinical practice may differ among individual patients.

\section{Target population}

The target groups of these guidelines are newly or previously diagnosed patients with hepatitis C virus (HCV) infection, including not only chronic hepatitis $C$ and cirrhosis but also acute hepatitis C patients, hepatitis C patients with chronic kidney diseases, and those patients coinfected with human immunodeficiency virus (HIV) or hepatitis B virus (HBV).

\section{Intended users}

The guidelines are intended to provide useful information and guidance to physicians and healthcare providers involved in the diagnosis and treatment of hepatitis $C$, and resident physicians, practitioners, and trainers.

\section{Development, funding, and revision process}

The Clinical Practice Guidelines Committee for the Management of Hepatitis C (Committee) comprising 14 hepatologists, was organized according to the proposal and approval of the KASL Board of Executives. Funding for the revision was provided by KASL. Each committee member collected and analyzed the source data in his or her own field, and the members then wrote the manuscript together.

\section{Literature review for evidence collection}

The committee systematically collected and reviewed the inter- national and domestic literature published in PubMed, MEDLINE, KoreaMed, and other databases. The key words used were 'hepatitis C virus', 'hepatitis C', 'liver cirrhosis', 'liver cancer' and other related specific key words.

\section{Levels of evidence and grades of recommendations}

The quality of evidence was classified according to the GRADE (Grading of Recommendations, Assessment, Development, and Evaluation) system (Table 1). ${ }^{1}$ Based on the types of study, randomized control studies were approached from a high level of evidence, while observational studies were approached from a low level of evidence. Then, the level of evidence was adjusted by accounting for the factors influencing the quality of the studies. Through follow-up studies, the level of evidence was defined as follows: $A$, the highest level of evidence with the smallest possibility of changes in the conclusion; $B$, a moderate level of potential changes; and $C$, the lowest level of evidence with the greatest possibility of changes.

The strength of a recommendation was also classified according to the GRADE system. Each study was classified as strong recommendation (1) or weak recommendation (2) based on the quality of evidence, the balance between the desirable and undesirable effect of an intervention, and socioeconomic aspects including cost or availability. A strong recommendation indicated that the interventions could be applied in most patients with strong certainty and that there was a greater possibility of desirable effects, high-quality evidence, and presumed patient-important outcomes, cost-effectiveness, preference, and compliance. A weak recommendation indicated a suggestion made with less certainty but that could be considered favorable for many patients, based on

Table 1. Grading of Recommendations, Assessment, Development, and Evaluation (GRADE)

\begin{tabular}{ll}
\hline Quality of evidence & Criteria \\
\hline High (A) & $\begin{array}{l}\text { Further research is very unlikely to change our confidence in the estimate of effect. } \\
\text { Moderate (B) }\end{array}$ \\
$\begin{array}{ll}\text { Further research is likely to have an important impact on our confidence in the estimate of effect and } \\
\text { may change the estimate. }\end{array}$ \\
$\begin{array}{ll}\text { Further research is very likely to have an important impact on our confidence in the estimate of effect } \\
\text { and is likely to change the estimate. Any change of estimate is uncertain. }\end{array}$ \\
\hline $\begin{array}{l}\text { Strength of Recommendation } \\
\text { Criteria }\end{array}$ \\
$\begin{array}{l}\text { Factors influencing the strength of the recommendation included the quality of the evidence, presumed } \\
\text { patient-important outcomes, and cost. }\end{array}$ \\
$\begin{array}{l}\text { Variability in preference and values, or more uncertainty. Recommendation is made with less certainty, } \\
\text { higher cost or resource consumption. }\end{array}$ \\
\hline
\end{tabular}

Note: Of the quality levels of evidence, we excluded "very low quality (D)" in our guideline for convenience, which was originally included in the GRADE system. 
the level of evidence, the cost or preferences of the patients or medical practitioners.

\section{List of key questions}

The revision committee considered the following clinical questions as the key components to be covered in these guidelines.

1. What is the epidemiology, natural history and prevention strategy of hepatitis C in Korea?

2. How should diagnosis and evaluation of severity of chronic hepatitis $\mathrm{C}$ be made?

3. What is the goal of treatment and who are the targets for antiviral treatment of hepatitis $\mathrm{C}$ ?

4. How to define the treatment response and what are the predictors of the response?

5. How to treat patients with genotype 1 chronic hepatitis $C$ and compensated cirrhosis?

6. How to treat patients with genotype 2 chronic hepatitis $C$ and compensated cirrhosis?

7. How to treat patients with genotype 3 chronic hepatitis $C$ and compensated cirrhosis?

8. How to treat patients with genotype 4 chronic hepatitis $C$ and compensated cirrhosis?

9. How to treat patients with genotypes 5 and 6 chronic hepatitis $C$ and compensated cirrhosis?

10. How to treat patients with decompensated cirrhosis?

11. How to treat patients who underwent liver or extrahepatic organ transplantation?

12. How to treat patients with acute hepatitis $\mathrm{C}$ ?

13. How to monitor the patients and the adverse effects of antiviral drugs during and after antiviral treatment?

14. How to treat patients with special conditions (people who inject drugs, chronic kidney diseases, coinfection with HIV or HBV, hemophilia or thalassemia, immunosuppressive therapy or cytotoxic chemotherapy, and pediatric patients)?

\section{Review of the manuscript and approval process}

Each manuscript written by committee members was reviewed, agreed, and approved through meetings of the committee. The quality of the manuscript was evaluated based on the standards suggested by AGREE II (Appraisal of Guidelines for Research and Evaluation II) along with the academic integrity of the contents. The guidelines were reviewed after counsel from an infection spe- cialist, at a meeting of an external review board composed of 14 KASL members, and were further modified following opinions aired at a public hearing, and a symposium open to all KASL members. The final manuscript was approved by the KASL Board of Executives.

\section{Release of the guidelines and plan for updates}

The Korean version of the KASL Clinical Practice Guidelines for the Management of Hepatitis C was released in November 2015 at a KASL meeting and published in January 2016 on the KASL website (http://www.kasl.org). Future plans for revision will be conducted under the judgment that the revision is necessary for promotion of health in South Korea with accumulation of research on the management of hepatitis C. In addition, use of DAA is to be allowed in South Korea in the near future, so that updating or partial revision of the guidelines, as appropriate, is warranted.

\section{EPIDEMIOLOGY}

$\mathrm{HCV}$ is one of the main causes of acute and chronic hepatitis, cirrhosis, and hepatocellular carcinoma. ${ }^{2}$ Hepatitis $C$ is on the list of National Notifiable Infectious Disease in South Korea and has

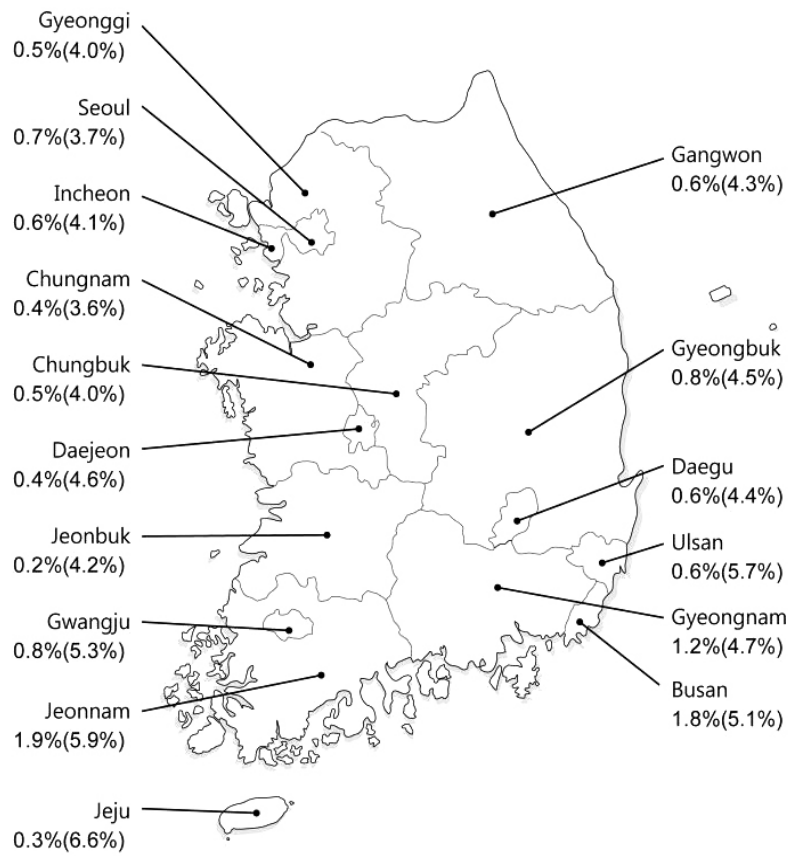

Figure 1. Map of South Korea showing age and sex-adjusted anti-HCV seroprevalence in each area." 
The Korean Association for the Study of the Liver (KASL) KASL clinical practice guidelines: Management of Hepatitis C

been under surveillance since 2000. An effective HCV vaccine is yet to be discovered, so that understanding of national epidemiology and a preventive strategy to block routes of HCV infection is important for public health.

\section{Prevalence of HCV infection}

The worldwide prevalence of HCV infection was 2.8\% in 2005 and $1.6 \%$ in 2014, equating to about 115 million persons positive for antibody to hepatitis C (anti-HCV) and 1.1\% equating to about 80 million persons positive for HCV RNA. ${ }^{3}$ The prevalence varies among geographic regions; regions with a high prevalence of over 3.5\% include Central Asia (including Mongolia and China), SouthEast Asia (including Pakistan and Thailand), and North Africa (including Egypt). Low prevalence (below 1.5\%) regions are Asia (including South Korea and Japan), North America (including the United States), and South America. ${ }^{4}$

\section{Prevalence in adult health check examinees}

The prevalace of HCV infection in adult health check examinees was reported to be $1.7 \%$ using a first-generation enzyme immunoassay (EIA) in the early 1990s soon after HCV was discovered. ${ }^{5}$ The estimated age-standardized prevalence of anti-HCV in adult health check examinees in $>40$ years of age was reported to be $1.29 \%$ (95\% confidence interval, 1.12-1.48) and was over 193,000 persons in a collective study of health checkup examinees from Seoul, Ulsan, Jeollanam-do, and Daegu between 1995 and 2000 . $^{6-10}$

In 2009, the anti-HCV prevalence in health examinations of 291,314 adults $\geq 20$ years of age from 29 health examination centers was $0.78 \%$ using third-generation ElA after adjusting for age, sex, and area." The anti-HCV prevalence was higher in females $(0.83 \%)$ than in males $(0.75 \%)$ and increased with age $(20-29$ years: $0.34 \%, 30-39$ years: $0.41 \%, 40-49$ years: $0.60 \%, 50-59$ years: $0.80 \%, 60-69$ years: $1.53 \%, \geq 70$ years: $2.31 \%)$. In addition, the anti-HCV prevalence varied geographically; in comparison with the prevalence of $0.50-1.20 \%$ in most regions, including Seoul and Gyeonggi-do, the prevalence in Pusan and Jeollanamdo were $1.53 \%$ and $2.07 \%$, respectively, while the Jeju Special Self-Governing Province had the lowest rate of $0.23 \%$ (Fig. 1). And also, there was a significant geographic difference in the potential risk factors for HCV infection in Korea. ${ }^{12}$ An update of the national HCV infection prevalence in South Korea is expected to be released at the end of 2015, and the Korea National Health and Nutrition Examination Survey has included anti-HCV testing since 2012.

\section{Prevalence of anti-HCV in blood donors, pregnant women, and children}

The anti-HCV prevalence in 2,040,151 blood donors in 1997 in South Korea was $0.34 \%$, as determined by third-generation EIA. ${ }^{13}$ From 2005 to 2009, the anti-HCV prevalence in 11,064,532 blood donors was $0.16 \%$, and the HCV RNA-positive rate was 8.4 $(0.0084 \%)$ of 100,000 donors, among whom $81 \%$ were young people aged 10-30 years. ${ }^{14}$ In South Korea, the risk of blood transfusion-related HCV infection decreased from 1 in 81,431 in 2000/2001 to 1 in 2,984,415 after implementation of nucleic acid testing for HCV screening of donated blood in February $2005 .^{15}$

The anti-HCV prevalence in pregnant women was reported to be $0.49-1.7 \%{ }^{16-18}$ and a domestic report investigating over 5,000 pregnant women reported rates of $0.42-0.44 \%{ }^{19,20}$ Among antiHCV-positive pregnant women, $57-60 \%$ of the patients positive for HCV RNA. ${ }^{19,20}$

Domestic studies on anti-HCV prevalence in children and adolescents are insufficient. A $0.82 \%$ anti-HCV-positive rate tested by third-generation EIA in 2,080 children between 6 and 11 years of age living in Seoul was reported. ${ }^{21}$ However there have been no other reports or studies on children and adolescents, hindering the accurate assessment of the HCV infection prevalence in the pediatric population of South Korea.

\section{Prevalence of anti-HCV in high-risk groups}

The high-risk groups for HCV infection include people who inject drugs (PWID), patients under hemodialysis, and those with HIV infection, hemophilia, and leprosy. However, the HCV prevalences in this group have been reported mostly before 2000; few studies were performed thereafter.

The domestic anti-HCV prevalence in the intravenous drug user (IVDU) group was 48.4-79.2\%. ${ }^{22-25}$ Among anti-HCV-positive persons, $98.1 \%$ were HCV RNA-positive. ${ }^{24}$ The anti-HCV prevalence in those who share cocaine suction pipes was similar to that in the IVDU group. ${ }^{26}$

The anti-HCV prevalence was $5.9-14.7 \%$ in previous studies of $>200$ patients with chronic kidney diseases, ${ }^{27,28} 2.2 \%$ in the 2014 report of the Korean Society of Nephrology ${ }^{29}$ and was significantly correlated with hemodialysis duration.

The HCV coinfection rate was high in those infected with HIV; $\sim 25 \%$ of westerners and $5.0-6.3 \%$ of HIV-infected individuals in South Korea were coinfected with $\mathrm{HCV}^{30-32}$

The anti-HCV prevalence in 104 hemophilia patients tested by 
third-generation EIA was $42.3 \%$ in 2002, and the risk of infection was correlated with age and severity of hemophilia. ${ }^{33}$ In their 2012 annual report, the Korea Hemophilia Foundation (KHF) reported that 430 of 2,148 (20.0\%) hemophilia patients were antiHCV-positive and 118 of 2,148 (5.5\%) were HCV RNA-positive. ${ }^{34}$

Leprosy patients can be considered at high risk of HCV infection due to their skin lesions and long-term cohabitation in limited areas. The anti-HCV prevalence of 96 leprosy patients tested by second-generation EIA was $67.7 \%$ in $1997 ; 82 \%$ of these individuals were immunoblot-positive. ${ }^{35}$

\section{HCV incidence rate}

Studies on HCV infection incidence rate are rare, since only 20 $30 \%$ of those with acute HCV infection develop symptoms. HCV incidence rates are decreasing in Western countries. ${ }^{36}$ In the US the incidence rate decreased from 7.4 of 100,000 people from 1982-1989 to 0.7 of 100,000 people from 1994-2006, ${ }^{37}$ and in Italy, it decreased from 2.02 of 100,000 people in 1996 to 0.55 of 100,000 people in $2006 .^{38}$

The HCV infection incidence rate in South Korean blood donors was reported to be 13.8 per 100,000 according to a survey conducted on those who donated blood at least twice from 19941996. ${ }^{39}$ The recent HCV infection incidence rates among blood donors who donated at least twice in 2 years between 2000 and 2010 were estimated to be 6.80 in 2001, 3.19 in 2003, 2.69 in 2005, 1.83 in 2007, and 0.80 in 2009 per 100,000 person-years, showing significant decrease in the incidence of HCV in this population in South Korea. ${ }^{15}$ According to surveillance sample data from the Korea Centers for Disease Control and Prevention $(K C D C)$, the number of reported hepatitis $C$ cases was 1,927 in 2002, 6,407 in 2008, and 4,280 in 2012. Future studies should evaluate the nationwide incidence of HCV in Korea.

\section{Distribution of HCV genotypes}

Globally, HCV genotypes 1, 2, and 3 are common and genotypes 4, 5, and 6 are localized to limited regions. ${ }^{40,41}$ Genotype 1a is the most common in Northern Europe and North America, and $1 \mathrm{~b}$ is the most common in Far East Asia and Europe. Genotype 2 is less common than genotype 1. Genotype 3 is common in Southeast Asia and genotype 4 is common in the Middle East, Egypt, and Central Africa. Genotype 5 is commonly found in South Africa and genotype 6 is common in Hong Kong, Macau, and Vietnam. Common HCV genotypes in South Korea are genotype $1 \mathrm{~b}(45-59 \%)$ and $2 \mathrm{a}$ (26-51\%); types $1 \mathrm{a}, 2 \mathrm{~b}, 3,4$, and 6 are rare in South Korea. ${ }^{42,43}$

Whether genotype $1 \mathrm{HCV}$ infection provokes faster progression of hepatic disease than the other genotypes is controversial. A recent meta-analysis reported that genotype $1 \mathrm{~b}$ patients showed a 1.78-fold higher risk of developing $\mathrm{HCC}(95 \% \mathrm{Cl}, 1.36-2.32)$ compared to non-1 genotype patients. ${ }^{44}$ Nevertheless, the HCV genotype is the most crucial factor in determining the efficacy of antiviral therapy. ${ }^{45}$

\section{PREVENTION}

\section{Route of transmission}

HCV transmission occurs by parenteral exposure. The main routes of transmission include transfusion of contaminated blood or blood products, organ transplantation, PWID, unsafe injection or medical procedures, stabs by contaminated syringe or needle, sexual contact with HCV-infected person, or perinatal transmission from an infected mother to her newborn.

Transmission via transfusion was a main route of infection until 1991, but the possibility has become extremely low since introduction of a screening test for blood donors. ${ }^{46-48}$ Recently, in developed regions such as the US and Europe which have low HCV prevalence, the most common route of HCV transmission has been the use of illicit drugs, ${ }^{49}$ and anti-HCV prevalence in the PWID group was reported to be up to $50-90 \%$. $^{50}$ Meanwhile, unsafe injection with multiple-use medication vials or reused syringes, or unsanitary medical procedures including surgery, endoscopy, and dental treatment without proper disinfection are the main causes of HCV transmission in developing countries. ${ }^{51-53}$ In addition, meta-analyses have reported that risk factors for HCV transmission include piercing, acupuncture, or tattooing without proper disinfection. ${ }^{54-56}$ The risk of HCV infection due to percutaneous exposure to a small dose, such as needle sticks, is 1.8\% $(0-7 \%)^{57-60}$ in other countries and $0.92 \%$ in South Korea. ${ }^{61}$ Heterosexual persons with chronic HCV infection in long-term monogamous relationships with a partner had little evidence of sexual transmission of HCV. However, the risk becomes higher with multiple sex partners, and unsafe sex including anal sex, sex accompanying wounds, sex with carriers of other sexually transmitted diseases such as HIV, or in homosexuals. ${ }^{62,63}$ The percentage of perinatal transmission was $1-6.2 \%{ }^{64,65}$ It was reported to be $1.7 \%$ when the mothers were positive for anti-HCV regardless of HCV RNA-positivity, and $4.3 \%$ (3.9-7.1\%) in the case of HCV RNA-positive mothers. ${ }^{65,66}$ The risk 
The Korean Association for the Study of the Liver (KASL) KASL clinical practice guidelines: Management of Hepatitis C

of perinatal transmission increased in female infants, HIV-positive mothers, and mothers with high blood HCV RNA levels. ${ }^{67}$ Cesarean section reportedly does not prevent HCV transmission, ${ }^{67,68}$ and the frequency of transmission via nursing was very low. Thus, it is not necessary to limit breast-feeding unless nipples are injured or bleeding. ${ }^{69}$ Reports of horizontal transmission between siblings or family members of HCV-infected persons are based on a low level of evidence. ${ }^{70}$

A comparative study of 1,173 HCV patients and 534 controls in five university hospitals between 2007 and 2011 in South Korea reported several independent risk factors of infection, including use of illicit drugs, needle-stick injury, transfusion before 1995, tattoo, and age. ${ }^{71}$

\section{Counseling for prevention}

Since an effective vaccine has not been developed, the main strategy for prevention is to educate people on the risk factors for HCV infection and to maintain strict sanitation standards in all locations performing percutaneous procedures.

HCV-infected persons should be counseled not to donate blood, organs, tissues, or semen, and not to share any instrument penetrating skin. They should use their own instruments including toothbrushes, oral hygiene devices, razors, or nail clippers so other people are not exposed to his/her blood. Finger-stabbing needles commonly used for Korean home remedies should not be shared. IVDU should be persuaded to stop drug abuse and they should not reuse syringes, needles, injection solution, cotton swab, or alcohol sponges. They must be reminded that other people can be infected via recklessly disposed needles. Since the risk of infection among monogamous couples is very low, use of barrier protection by these couples is not necessarily recommended. Nevertheless, if the partner of the infected individual requests, or if the infected person has multiple sex partners, it is recommended to use condoms. Routine screening for HCV is not recommended for all pregnant women. However, for those with a risk factor, prenatal testing for $\mathrm{HCV}$ is required. $\mathrm{HCV}$ infection does not mean a restriction of breast-feeding or recommendation for a specific means of delivery, such as Cesarean section. Healthcare facilities should take precautions to prevent HCV transmission. Proper disinfection, cleaning, and management of materials and instruments are essential in medical and invasive procedures including tattooing, piercing, and acupuncture.

\section{[Recommendations]}

1. HCV-infected persons should not donate blood, organs, tissues, or semen (A1). HCV-infected persons should avoid sharing toothbrushes, oral hygiene devices, razors, nail clippers, or any instrument penetrating skin, so as not to expose other people to his/her blood (C1).

2. People who inject drugs should be counseled to stop abuse of illicit drugs (A1). They should be educated about routes of infection and tested regularly for HCV infection (B1).

3. Proper disinfection, cleaning, and management of materials and instruments are essential in medical and invasive procedures including tattooing, piercing, and acupuncture (B1).

4. As the risk of infection among monogamous sexual partners is very low, use of barrier protection is not advised in these couples (B1). However, for those with multiple sex partners, it is recommended to use condoms (B1).

5. For pregnant women, if a risk factor for HCV infection is detected or HCV infection is suspected otherwise, prenatal testing for HCV infection is recommended (B1). HCV infection does not mean a restriction of breast-feeding or a recommendation of specific delivery, such as Cesarean section (B2).

\section{NATURAL HISTORY}

\section{Acute HCV infection}

After 1-3 weeks of HCV infection, HCV RNA becomes detectable in blood and the level rapidly increases. ${ }^{72,73}$ The serum alanine transaminase (ALT) level increases due to hepatocyte damage after 4-12 weeks of infection. Most infections are asymptomatic (70$80 \%$ ), but symptoms including flu-like symptoms, fatigue, vomiting, nausea, right upper quadrant pain, muscle pain, or pruritus may develop within 2-12 weeks. About $20 \%$ of acute infection accompanies jaundice with serum bilirubin level $<3-8 \mathrm{mg} / \mathrm{dL}$, and acute liver failure occurs in $<1 \%$ of cases.

Acute hepatitis progressed to chronic infection in $54-85 \%$ of patients, and $20-50 \%$ of patients recovered spontaneously within 3-4 months. ${ }^{74-76}$ The spontaneous recovery rate differs according to route of infection; the spontaneous recovery rate in post-transfusion cases was $12 \%$, while in the cases not related to transfusion it was $29-52 \%{ }^{76-78}$ Factors related to spontaneous recovery are hepatitis accompanying jaundice, female gender, low viral 
load, and genotype $3{ }^{77-79} \mathrm{~A}$ Korean study reported that among 18 acute hepatitis C patients (17 patients showed symptoms), 12 patients spontaneously recovered and 6 patients progressed to chronic hepatitis. ${ }^{80}$ Another study including 47 acute hepatitis C patients with a mean age of 45.8 years in seven Korean institutions reported that 21 of $47(44.7 \%)$ patients recovered spontaneously, and 16 patients received antiviral therapy. All 12 patients who were treated and followed-up achieved a sustained virological response (SVR). Ten patients who did not receive antiviral therapy progressed to chronic hepatitis. ${ }^{81}$

A single nucleotide polymorphism (SNP) of the interleukin 28B (IL28B) gene is strongly related to spontaneous recovery from acute hepatitis C infection. ${ }^{82-84}$ IL28B is located on chromosome 19 and encodes interferon-lamda-3. One study reported a spontaneous recovery rate of 53\% in cases with genotype CC of IL28B SNP rs 12979860 and of $28 \%$ in genotype CT or TT (Odds ratio $(\mathrm{OR})=0.33, P<10-12) .{ }^{85}$ However, future studies are needed as there has been no Korean study of the role of IL28B SNP in acute HCV infection.

\section{Chronic HCV infection}

About $50-80 \%$ of HCV-infected patients progress to chronic infection. Chronic hepatitis can cause persistent liver injury without spontaneous recovery, leading to cirrhosis and HCC. Most (60$80 \%$ ) patients with chronic hepatitis show no symptoms, but some can experience abdominal discomfort, fatigue, nausea, muscle pain, arthritis, or weight loss. About $60-70 \%$ of chronic HCV-infected patients show chronic hepatitis accompanying steady or intermittent elevation of serum ALT. About $15-56 \%$ of chronic hepatitis may progress to cirrhosis through a period of 2025 years. ${ }^{73,86-88}$ Among patients with liver cirrhosis, the annual incidence of HCC is reported as 1-4.9\%, ${ }^{89-91}$ that of decompensated liver cirrhosis is 3-6\%, ${ }^{73,90-92}$ and the overall annual mortality rate is 2-4\%. ${ }^{73,90-92}$ An observational study including 1,137 Korean chronic HCV infected patients for an average follow-up of 55.2 months reported a $14.2 \%$ rate of disease progression, defined as development of HCC, spontaneous bacterial peritonitis, variceal bleeding, hepatic encephalopathy, and death due to hepatic diseases, and the overall annual mortality rate was $2.0-2.5 \% .{ }^{93}$ The cumulative probability of disease progression was $6.3 \%, 12.9 \%$, and $26.1 \%$ at 1,2, and 3 years, respectively. Among 1,137 patients, $490(43.0 \%)$ received antiviral treatment and $60.4 \%$ showed an SVR. Chronic infection without antiviral treatment showed a significantly higher risk of disease progression com- pared to chronic infection with antiviral treatment (37.4\% vs. $10.7 \%$, respectively, $P<0.05$ ). The 5 -year cumulative probability of disease progression was higher in a non-SVR group compared to a group with SVR $(13.0 \%$ vs. $3.7 \%$, respectively, $P<0.05)$. According to prospective cohort data from 196 patients with HCVrelated cirrhosis in Korea, during a mean follow-up period of 39.2 months, 31 (15.8\%) patients developed HCC, and 33 (16.8\%) patients died or underwent liver transplantation. The estimated HCC incidence was 5.8 per 100 person-years, and the independent factors for HCC were absence of anti-HBV surface antibody (HBS hazard ratio $[\mathrm{HR}], 5.018 ; 95 \%$ confidence interval $[\mathrm{Cl}], 1.710$ 14.726; $P=0.003$ ) and serum albumin $<3.8 \mathrm{~g} / \mathrm{dL}$ (HR, 3.051; 95\% $\mathrm{Cl}, 1.318-7.067 ; P=0.009)$. The overall mortality rate was 5.1 per 100 person-years, and the related independent factors were the presence of ascites (HR, 2.448; 95\% Cl, 1.142-5.210; $P=0.022$ ), serum albumin $<3.8 \mathrm{~g} / \mathrm{dL}(\mathrm{HR}, 3.067 ; 95 \% \mathrm{Cl}, 1.254-8.139$, $P=0.014)$, and nonachievement of SVR (HR, $0.066 ; 95 \% \mathrm{Cl}$, $0.001-0.484, P=0.002) .{ }^{94}$

Factors affecting disease progression include duration of infection, age at the time of infection ( $\geq 40$ years of age), male gender, alcohol intake, coinfection with other viruses (HBV or HIV), insulin resistance, obesity, immune-depressed patients, organ transplantees, elevation of ALT, and genetic factors such as IL28B. ${ }^{73}$ Excessive alcohol intake by chronic hepatitis $C$ patients is strongly related to occurrence of cirrhosis, and increases the risk of $\mathrm{HCC}^{88,95-98}$ Fatty liver, insulin resistance, and obesity increase the risks of hepatic fibrosis and HCC development in chronic hepatitis C patients. ${ }^{99-102}$ Coinfection with HIV or HBV accelerates progression of liver diseases and increases the risk of HCC compared to HCV single infection. ${ }^{103-105}$ In addition, coinfection with hepatitis A virus (HAV) in chronic hepatitis $C$ increases the risk of hepatic failure. ${ }^{106}$ The pathologic stage of hepatic fibrosis at the time of chronic hepatitis $\mathrm{C}$ diagnosis is the most important predictor of progression to cirrhosis (refer to the Diagnosis section of this paper). ${ }^{88,103}$ Stage 1 hepatic fibrosis has a 10-30\% incidence of cirrhosis over a period of 15 years, while most cases of stage 3 hepatic fibrosis are expected to progress to cirrhosis within 15 years. Therefore, patients diagnosed as having hepatic fibrosis over stage 2 must be considered for active antiviral treatment.

\section{[Recommendations]}

\section{Continuous management and surveillance for development of cirrhosis and HCC is necessary in chronic hepatitis C pa- tients (A1).}


The Korean Association for the Study of the Liver (KASL)

Table 2. High-risk persons recommended for HCV infection screening
1) Persons who are suspected of having acute or chronic HCV infection
2) Persons who have received blood/blood products transfusions or organ transplants prior to screening program
3) Persons who have ever injected illicit drugs
4) Persons who have ever been on hemodialysis
5) Persons with HIV infection
6) Persons with hemophilia
7) Persons who have current sexual contact with HCV-infected persons ${ }^{*}$
8) Children born to mothers infected with HCV
9) Health care providers after a needle stick injury or mucosal exposure to HCV positive blood
NOTE: Table adapted from Diagnosis, Management and Treatment of Hepatitis C Hepatology 2009;49:1335-1374. ${ }^{107}$
$\mathrm{HCV}$, hepatitis $\mathrm{C}$ virus.
"The prevalence of infection is low.

Table 3. Interpretation of HCV assays

\begin{tabular}{|c|c|c|c|}
\hline Anti-HCV & HCV RNA & Interpretation & Further evaluation \\
\hline \multirow[t]{2}{*}{ Positive } & Positive & Acute hepatitis C & \\
\hline & & Chronic hepatitis C & \\
\hline \multirow[t]{4}{*}{ Positive } & Negative & Resolution of HCV infection & Recheck anti-HCV \& HCV RNA, 3-6 months later \\
\hline & & Acute HCV infection during period of low-level viremia & \\
\hline & & False positive anti-HCV test & \\
\hline & & False negative HCV RNA test & \\
\hline \multirow[t]{3}{*}{ Negative } & Positive & Early acute HCV infection & Recheck anti HCV \& HCV RNA, 3-6 months later \\
\hline & & Chronic HCV infection in setting of immunosuppressed state & \\
\hline & & False positive HCV RNA test & \\
\hline
\end{tabular}

NOTE: Table adapted from Diagnosis, Management and Treatment of Hepatitis C Hepatology 2009;49:1335-1374. ${ }^{107}$

$\mathrm{HCV}$, hepatitis C virus.

2. Chronic hepatitis $C$ patients should abstain from alcohol or drink in moderation, and maintain suitable body weight through physical exercise and dietary control, since disease progression is related to alcohol, obesity, and insulin resistance (B1).

3. Patients with chronic HCV infection without antibodies against HAV and HBV should be vaccinated for HAV and HBV (C1).

\section{Screening test for HCV infection}

Routine screening for HCV infection is recommended in populations at risk, such as those with a history of blood transfusions or organ transplantation prior to 1992; persons who have injected illicit drugs; persons with HIV infection, hemophilia, or Hansen's disease; persons who have been on hemodialysis; children born to mothers infected with $\mathrm{HCV}$; and health care providers after a needle stick injury or mucosal exposure to HCV-positive blood (Table 2). ${ }^{107}$ In 2012, the US Centers for Disease Control and Prevention expanded the screening population to the birth cohort born between 1945-1965 and recommended screening for HCV once in a lifetime, based on cost effectiveness. ${ }^{108-110} \mathrm{~A}$ Japanese study revealed that hepatitis C screening appears cost-effective in the general population as well as in high-risk groups, ${ }^{111}$ but a European study showed that screening of the general population is cost-effective only in HCV prevalent areas. ${ }^{112}$ Therefore, since the epidemiologic characteristics and healthcare systems differ among nations, further research on cost effectiveness is needed to determine the optimal screening strategy for Korea.

According to anti-HCV prevalence rates in Korea, the estimated number of anti-HCV infected persons over 20 years old was about 320,000 in 2009 . However, the number of patients who were 
treated under coverage of Health Insurance Review and Assessment Service (HIRA) due to liver disease associated with HCV infection was 64,501 in $2009 . .^{113}$ These data suggest the possibility that only about $20 \%$ of the patients were diagnosed and treated. An online survey in 2013 about awareness of HCV infection showed that about $90 \%$ of the general population had not been tested for hepatitis $\mathrm{C}$ or did not know whether they had been tested for hepatitis $C$, which implies that awareness of HCV infection in the general population was low. According to HIRA data, the direct annual medical cost per patient in South Korea was markedly higher for liver cirrhosis (1,522 USD), hepatocellular carcinoma (6046 USD) or liver transplantation (57,940 USD) compared to in chronic hepatitis (842 USD). ${ }^{113}$ Such results demonstrate that every effort to intervene disease progression should be exerted to reduce the HCV disease burden. The high accuracy rate and low cost of testing for anti-HCV as a screening tool, and the $>90 \%$ cure rate of DAA in early stage liver disease, should also be taken into consideration. One-time screening for HCV infection in the South Korean population aged 40-70 years is likely to be more cost-effective compared to current practice. ${ }^{114}$ Therefore, a nationwide screening and therapeutic strategy to diagnose and cure hepatitis C patients prior to progression to advanced liver disease would not only decrease the disease burden but also be a successful model for HCV elimination.

\section{Diagnosis of HCV infection}

Biochemical tests, serologic assays, and HCV RNA testing are needed to confirm HCV infection. Physical examination and history taking should be performed to understand the routes of transmission and prevent further reinfection. HCV genotyping is essential for treatment, and radiologic examination, liver biopsy, or noninvasive evaluation of hepatic fibrosis can be performed to determine the necessity of treatment, and to assess liver disease severity. Interpretation of serological and virological test results is summarized in Table 3.

\section{DIAGNOSIS}

\section{Serologic assays: Anti-HCV test}

Detection of anti-HCV in serum or plasma is used for screening of high-risk groups and for diagnosis of acute or chronic hepatitis $\mathrm{C}^{115}$ The third-generation EIA uses recombinant core, NS3, NS4, and NS5 HCV proteins, and its sensitivity and specificity are 97.299\% and 99.8-100\%, respectively, when tested in immune-competent individuals. ${ }^{116-118}$ If signal/cutoff $(\mathrm{S} / \mathrm{CO})$ ratios of third-generation EIA exceed 3.8, a positive result will be apparent in 95\% of recombinant immunoblot assays (RIBA). ${ }^{119-121}$ However, the cutoff S/CO ratio can differ according to the type of equipment, so that high S/CO ratios do not always mean true positive. ${ }^{122}$ Recently, use of enhanced chemiluminescent immunoassay (CLIA) or electrochemiluminescence immunoassay (ECLIA) is increasing since those assays detect antigen-antibody reaction more sensitively compared to the third-generation EIAs. Meanwhile, pointof-care tests using saliva or fingerstick blood that produce results within 20 minutes can also be employed. ${ }^{123,124}$

Average time between HCV infection and seroconversion is 8-9 weeks, and anti-HCV is detectable in $>97 \%$ of patients with HCV infection within 6 months. ${ }^{107,125}$ Anti-HCV is not a neutralizing antibody and persists indefinitely in chronic hepatitis $C$ patients and after recovery. Therefore, the differentiation of current from past infection after recovery is impossible using anti-HCV positivity. Anti-HCV can be tested repeatedly in high-risk persons because hepatitis $C$ virus can reinfect after recovery. Negative result for anti-HCV in combination with a positive result for HCV RNA may represent an early stage of acute infection or chronic infection in the setting of severe immunosuppression, such as patients on hemodialysis, HIV coinfection, solid organ transplantation recipients, hypo-/ a-gammaglobulinemia, and patients with HCV-associated essential mixed cryoglobulinemia. ${ }^{126-128}$ In these patients, HCV RNA testing is necessary for diagnosis of HCV infection. In contrast, false-positive result for anti-HCV and negative result for HCV RNA can occur in patients with autoimmune diseases. ${ }^{129}$

\section{Virological assays}

\section{HCV RNA assays}

HCV RNA assays are classified as quantitative and qualitative. Since the detection cutoff of qualitative assays is $50 \mathrm{IU} / \mathrm{mL}$, and these are more sensitive than previous-generation quantitative assays, HCV RNA qualitative assays have been used for diagnostic confirmation of HCV infection, and HCV RNA quantification is used for pretreatment assessment and monitoring of a virological response during and after antiviral therapy. ${ }^{115,121,130}$ However, recently available quantitative HCV RNA assays use real-time polymerase chain reaction (PCR) and transcription-mediated amplification (TMA), and are highly sensitive with lower detection limits of $12-15 \mathrm{IU} / \mathrm{mL}$, while they have a broad measuring range with an 
The Korean Association for the Study of the Liver (KASL) KASL clinical practice guidelines: Management of Hepatitis C

Table 4. HCV drug resistance-associated variants

\begin{tabular}{|c|c|c|}
\hline Target enzyme & Drug & Mutation \\
\hline \multirow[t]{4}{*}{ NS3/4 Protease inhibitor ${ }^{156-164}$} & Boceprevir & V36, T54, V55, Q80, S122, R155, A156, D168 \\
\hline & Telaprevir & \\
\hline & Simeprevia & \\
\hline & Asunaprevir & \\
\hline \multirow[t]{2}{*}{ NS5A inhibitors ${ }^{156-159,164-166}$} & Daclatasvir & L28, Q30, L31, Y93 \\
\hline & Ledispasvir & \\
\hline NS5B Polymerase inhibitor ${ }^{164}$ & Sofosbuvir & S282, M289, C316, L320 \\
\hline
\end{tabular}

upper limit of 7-8 log $\mathrm{IU} / \mathrm{mL}$ and $98-99 \%$ diagnostic specificity irrespective of HCV genotype. ${ }^{115,131-135}$ Therefore, quantitative HCV RNA tests are now widely used for both diagnosis and evaluation of the treatment response. ${ }^{127,136}$

In 1997, the World Health Organization established an international standard for HCV RNA quantification unit, IU, rather than HCV copy number. ${ }^{137,138}$ However, since viral quantification results can differ among laboratories, ${ }^{139}$ it is recommended to use the same laboratory test before, during, and after-treatment for monitoring, if possible. ${ }^{107,127}$

Blood HCV RNA is detectable as early as 2 weeks after infection, ${ }^{76}$ rapidly increases to reach a plateau, and decreases along with ALT after ALT has peaked. ${ }^{140}$ HCV RNA levels remain steady in patients with chronic hepatitis $C^{140,141}$ HCV RNA levels are not significantly correlated with the severity of hepatic inflammation or fibrosis, and change little during chronic infection without antiviral treatment. ${ }^{142,143}$

\section{Genotyping/subgenotyping assays}

HCV genotyping is useful for epidemiologic studies as well as for predicting treatment response. Therefore, HCV genotype should be assessed before treatment to determine the optimal therapeutic duration and dose of ribavirin. ${ }^{144} \mathrm{HCV}$ is classified into six major genotypes (1-6) and is subdivided into subtypes identified by lower-case letters, such as 1a or $1 \mathrm{~b}$. Differences of 31 $33 \%$ at the nucleotide level differentiate the genotypes, compared with $20-25 \%$ for the subtypes. ${ }^{145}$ HCV genotype does not change within a person unless reinfected.

HCV genotypes and subtypes can be determined by direct sequence analysis, reverse hybridization, or restriction fragment mass polymorphism (RFMP). ${ }^{146}$ Most genotyping assays analyze both the $5^{\prime}$-untranslated region (UTR) and HCV core regions, the nucleotide sequences of which are highly conserved. ${ }^{147-150}$ Subtyping is not necessary in antiviral therapy using interferon alpha and ribavirin, but in treatment that includes DAAs, subtypes may need to be confirmed since DAAs act differently according to subgenotype. $^{148-153}$ Genotyping is not possible in $<5 \%$ of patients, because of low HCV RNA levels, problems with PCR amplification, or the high nucleotide variability of the HCV genome. ${ }^{154}$

\section{HCV drug-resistance mutation tests}

As various DAAs began to be used for therapy, amino acid sequence variants associated with resistance to DAA have been found (Table 4). ${ }^{155-166} \mathrm{HCV}$ resistance-associated variants (RAV) can be selected during the HCV life cycle, or during DAA therapy. Naturally occurring RAV has been found more commonly in HCV 1a than in HCV $1 b^{167}$ RAV to protease inhibitors (PI) were found in $9-48 \%$ of patients infected with HCV 1 a and $0.5-4.9 \%$ of those with HCV $1 b{ }^{168-170}$ while RAV to daclatasvir, an NS5A inhibitor, are found in $11.2 \%$ of patients infected with HCV $1 \mathrm{~b}$. RAVs to both NA5A inhibitor and NS3/4 PI are found in only $0.4 \%$ of patients. ${ }^{170}$ Asunaprevir-resistant substitutions found during therapy can disappear within a few weeks, but daclatasvir-resistant substitutions can persist up to 48 weeks post-treatment. ${ }^{160}$ Therefore, patients who failed the first DAA therapy should be retreated with drugs showing no cross-resistance with the drugs already administered. ${ }^{171}$ RAVs can be detected using several methods, including population sequencing, clonal sequencing and deep sequencing, by which variants with frequencies less than approximately $25 \%$, $5 \%$ and $0.5 \%$ respectively, cannot be detected. ${ }^{172}$ HCV RAV testing prior to first-line therapy is not generally required. However, the Q80K polymorphism should be tested in HCV 1a-infected patients who were previously treated with boceprevir or telaprevir, and will be treated with a simeprevir-containing regimen. In patients with HCV genotype 1a who have the Q80K variant, simeprevir is not recommended. In HCV 1b patients with baseline NS5A polymorphisms such as $\mathrm{L} 31$ or $\mathrm{Y} 93 \mathrm{H}$, daclatasvir plus asunaprevir combination therapy showed a significantly lower SVR 
Table 5. Comparison of scoring systems for histological stage

\begin{tabular}{|c|c|c|c|}
\hline Stage & Metavir $^{180}$ & Ishak ${ }^{181}$ & $\begin{array}{c}\text { Korean Study Group for } \\
\text { the Pathology of Digestive } \\
\text { Diseases }{ }^{182}\end{array}$ \\
\hline 0 & No fibrosis & No fibrosis & No fibrosis (F0) \\
\hline 1 & $\begin{array}{l}\text { Periportal fibrotic } \\
\text { expansion }\end{array}$ & $\begin{array}{l}\text { Fibrous expansion of some portal areas with or without short } \\
\text { fibrous septa }\end{array}$ & Portal fibrosis (F1) \\
\hline 2 & $\begin{array}{l}\text { Periportal septae } \\
1 \text { (septum) }\end{array}$ & $\begin{array}{l}\text { Fibrous expansion of most portal areas with or without short } \\
\text { fibrous septa }\end{array}$ & Periportal fibrosis (F2) \\
\hline 3 & Porto-central septae & $\begin{array}{l}\text { Fibrous expansion of most portal areas with occasional portal to } \\
\text { portal bridging }\end{array}$ & Septal fibrosis (F3) \\
\hline 4 & Cirrhosis & $\begin{array}{l}\text { Fibrous expansion of most portal areas with marked bridging } \\
\text { (portal to portal and portal to central) }\end{array}$ & Cirrhosis (F4) \\
\hline 5 & & $\begin{array}{l}\text { Marked bridging (portal to portal and portal to central) with } \\
\text { occasional nodules (incomplete cirrhosis) }\end{array}$ & \\
\hline 6 & & Cirrhosis & \\
\hline
\end{tabular}

NOTE: Table adapted from Diagnosis, Management and Treatment of Hepatitis C Hepatology 2009;49:1335-1374. ${ }^{107}$

than that in those without NS5A RAVs. Therefore, HCV NS5A RAV testing should be performed prior to treatment of HCV patients with daclatasvir plus asunaprevir. Otherwise, HCV RAV testing is not recommended during DAA therapy. ${ }^{173}$

\section{Diagnosis in case of accidental exposure}

The average incidence of anti-HCV seroconversion in healthcare providers after accidental percutaneous exposure to HCV-infected blood is $1.8 \%(0-7 \%)$ in various countries, ${ }^{59,60,174-178}$ and $0.92 \%$ in South Korea. ${ }^{61}$ When a person is exposed to an HCV-positive source, baseline testing for anti-HCV and serum ALT level should be performed. If anti-HCV is negative, HCV RNA assay should be performed 4- 6 weeks after exposure for early diagnosis. Even if all baseline tests for HCV infection are negative, follow-up testing for anti-HCV and serum ALT level should be performed 4-6 months after exposure. ${ }^{127,175}$ If anti-HCV is positive, a confirmative test is needed.

\section{Assessment of liver disease severity}

To decide the treatment for HCV-infected patients, the severity of liver disease must be evaluated through liver biopsy and/or noninvasive tests. It is important to confirm whether the patient has liver cirrhosis or not before treatment since the existence of liver cirrhosis can affect the treatment response, prognosis, and necessity of surveillance for HCC. Liver cirrhosis can be diagnosed using clinical characteristics, histologic findings and/or noninva- sive tests for evaluation of liver fibrosis.

\section{Liver biopsy}

Liver biopsy is performed to assess the grade and stage of the hepatic injury. ${ }^{107,179}$ The Metavir ${ }^{180}$ and Ishak ${ }^{181}$ scoring systems are most widely used, and the scoring system proposed by the South Korean Study Group for the Pathology of Digestive Diseases ${ }^{182}$ is used in South Korea (Table 5). Although liver biopsy is not mandatory prior to treatment, it can help to determine when to start treatment and to provide information regarding the treatment response and prognosis. Considering the natural history of the disease, the cost of treatment and its possible adverse effects, treatment can be postponed if liver histopathology shows minimal to moderate fibrosis, state $<2$ (Metavir stage 2 or periportal fibrosis of the South Korean Study Group for the Pathology of Digestive Diseases, F2). ${ }^{76,183,184}$ In this case, a liver biopsy should be repeated 4-5 years later to reassess the necessity of treatment according to the progression of liver disease. ${ }^{185}$ About $5-30 \%$ of patients with genotype 1 with consistently normal serum ALT may have severe fibrosis $^{186-188}$ and liver biopsy would be useful to determine treatment initiation in this group. ${ }^{88,189,190}$ Although hepatic steatosis ${ }^{179,191,192}$ and liver iron load overload ${ }^{193}$ might impede the treatment response, these findings are not contraindications for treatment. ${ }^{194-196}$ If a liver biopsy is not conducted and treatment is not undertaken, continuous monitoring is needed. Liver biopsy and treatment initiation should be considered when there is elevation of the serum ALT level and evidence of liver disease progression. ${ }^{107}$ 
The Korean Association for the Study of the Liver (KASL) KASL clinical practice guidelines: Management of Hepatitis C

ing prevalence of $\mathrm{HCV}$ infection (C1).

\section{Noninvasive tests for evaluation of liver fibrosis}

Although liver biopsy is accepted as the gold standard test for evaluation of liver fibrosis, ${ }^{197,198}$ it is associated with serious complications, ${ }^{199,200}$ sampling errors, ${ }^{201}$ high costs, and interobserver variations. Therefore, various blood marker panels have been developed-including aspartate aminotransferase (AST)-platelet ratio index (APRI) and AST/ALT ratio (AAR), and Fibrosis-4 score (FIB-4) - that use combinations of AST, ALT, and platelet count. FibroTest, Hepascore, FibroMeter, Fibrospect II, and Enhanced Liver Fibrosis tests can also be used. ${ }^{202-213}$

APRI is calculated by the formula (AST/upper limit of normal for AST) $\times 100 /$ platelet count $\left(\times 10^{9} / L\right)$ (www.kasl.org). This formula is accurate for predicting both significant fibrosis (Ishak score $\geq 3$ ) defined as APRI $>1.5(A U R O C=0.8)$ and cirrhosis defined as $A P R I>2(A U R O C=0.89) .{ }^{214} A$ normal value of $A A R$ is $<0.8$, but it increases with hepatic fibrosis progression, so that an $A A R$ value $>1.0$ has a $73.7-100 \%$ positive predictive value for diagnosis. ${ }^{207,215-217}$ FIB-4 is calculated using the formula: age $(\mathrm{yr}) \times$ AST (IU/ L)/platelet count $\left(10^{9} / \mathrm{L} \times\left[\right.\right.$ ALT $\left.(\text { IU/L })^{1 / 2}\right]$ (www.kasl.org). A FIB-4 score $<1.45$ has a negative predictive value of $90 \%$ for advanced fibrosis (Ishak fibrosis score 4-6). In contrast, a FIB-4 score of $>3.25$ has a positive predictive value of $65 \%$ for advanced fibrosis. ${ }^{218}$

Liver stiffness measurement using transient elastography can be used to assess hepatic fibrosis. ${ }^{219-222}$ However, transient elastography cannot totally replace liver biopsy, because it often cannot produce reliable measurements in obese patients, and tends to give falsely high results in cases of acute hepatitis with severe inflammation and necrosis with mild fibrosis. ${ }^{223,224}$ In the case of chronic hepatitis $C$, cutoff values determining significant fibrosis $(\geq F 2)$ vary among studies, ranging from 7.1 to $8.8 \mathrm{kPa}$, with an AUROC of 0.79 $0.83 .^{225}$ The AUROC for diagnosis of liver cirrhosis ranged from 0.95 0.97 , with cutoff values of $12.5-14.6 \mathrm{kPa}$ (77-78\% positive predictive value, $95-97 \%$ negative predictive value). ${ }^{202,209,219,225-228}$

Other newly developed noninvasive tests include acoustic radiation force impulse (ARFI) imaging, real-time elastography, magnetic resonance (MR) elastography, diffusion-weighted MR image, and MR spectroscopy. However, their effectiveness remains to be validated. $^{229-231}$

\section{[Recommendations]}

1. Screening for HCV infection could be considered in populations at risk as well as those over 40 years old with increas-
2. Anti-HCV should be tested in patients suspected of having acute or chronic HCV infection (A1).

3. HCV RNA should be tested in patients with a positive antiHCV test to confirm the diagnosis (A1).

4. Even if anti-HCV is negative, HCV RNA testing is required when acute HCV infection is suspected or in the presence of unexplained liver disease in immunosuppressed patients (B1).

5. HCV RNA quantitative assay and genotyping/subgenotyping (1a/1b) should be performed prior to antiviral treatment (A1).

6. Immediately following exposure to infected blood or body fluids, anti-HCV and serum ALT level testing should be performed. If anti-HCV is negative, an HCV RNA assay should be conducted 4-6 weeks after exposure for early diagnosis. If all baseline tests are negative, follow-up testing for antiHCV and serum ALT level should be performed 4-6 months after the exposure (B2).

7. Assessment of liver disease severity is essential prior to antiviral treatment (A1).

8. Liver biopsy and/or noninvasive tests for assessment of hepatic fibrosis can be performed to make treatment decision and predict prognosis (B1).

\section{TREATMENT GOALS}

The goals of hepatitis C treatment are to eradicate HCV and to prevent complications of liver cirrhosis, hepatocellular carcinoma, extrahepatic manifestations of HCV infection and death. It is difficult to evaluate the treatment goal in a short period of time due to the gradual progression of chronic hepatitis $C$ over several decades. Therefore, the short-term goal of hepatitis $C$ treatment is to achieve an SVR, defined as undetectable serum HCV RNA by a sensitive assay 12 or 24 weeks after the end of treatment. SVR was determined 24 weeks after the end of treatment using an assay with a lower limit of detection of $<50 \mathrm{IU} / \mathrm{mL}$ in studies of combination therapy with Peginterferon (PegIFN)- $\alpha$ and ribavirin, $^{232}$ whereas it was determined 12 weeks after the end of treatment using an assay with a lower limit of detection of 10-25 IU/ $\mathrm{mL}$ in studies on DAA therapy. ${ }^{233,234}$ Since HCV does not reappear in $99 \%$ of patients who achieve an SVR, ${ }^{232}$ the SVR is considered as eradication of HCV. Histological hepatic fibrosis improves or does not get worse, ${ }^{235,236}$ complications of cirrhosis significantly decrease, ${ }^{237}$ occurrence of hepatocellular carcinoma decreases, ${ }^{238,239}$ and survival rate improves. ${ }^{240,241}$ SVR also improves the 
extrahepatic manifestations of HCV infection, such as mixed cryoglobulinemia and glomerulonephritis. ${ }^{242,243}$

\section{[Recommendations]}

1. The goals of hepatitis $C$ treatment are to eradicate $H C V$ and to prevent complications of liver cirrhosis, hepatocellular carcinoma, extrahepatic manifestations of HCV infection and death (A1).

2. A short-term goal of hepatitis $C$ treatment is to achieve an SVR, defined as an undetectable serum HCV RNA using a sensitive assay at 12 or 24 weeks after the end of treatment (A1).

\section{INDICATIONS FOR TREATMENT}

All hepatitis C patients who have no contraindications to treatment can be considered for antiviral treatment. However, treatment would be applicable in cases in which the benefits of treatment outweigh the risks. Generally, treatment is strongly recommended for patients with significant hepatic fibrosis ( $\geq$ stage F2). Treatment for patients with advanced fibrosis (stage F3-4) who are at high risk of cirrhotic complications and hepatocellular carcinoma should be prioritized. ${ }^{90}$ Treatment of patients in the pre- and post-liver transplant setting should be a priority as successful eradication of HCV increases patient and graft survival. ${ }^{244,245}$ Treatment of patients with HCV-related mixed cryoglobulinemia and glomerulonephritis should also be a priority since HCV eradication can improve the prognosis of those patients. ${ }^{242,243}$ With advent of DAA, patients with decompensated liver cirrhosis (LC), which is a contraindication to the combination of PegIFN- $\alpha$ and ribavirin are candidates for antiviral treatment. HCV eradication can reduce the recurrence of hepatocellular carcinoma (HCC) and improve survival in patients with HCC. ${ }^{246,247}$ Therefore, patients with HCC can be treated using the rules applicable to those without $\mathrm{HCC}$, taking into consideration the prognosis of $\mathrm{HCC}$ and life expectancy.

In cases of mild hepatic fibrosis, treatment can be determined after considering patients' age, willingness to undergo treatment, and perspectives regarding new drugs.

Contraindications of DAA are limited due to the few adverse effects. Sofosbuvir is contraindicated in patients with several renal impairment (Glomerular filtration rate $[G F R]<30 \mathrm{~mL} / \mathrm{min}$ ) in whom the serum sofosbuvir concentration is markedly increased. Safety of daclatasvir, ledipasvir, and asunaprevir has not been fully evaluated in patients with severe renal impairment (GFR $<30$ $\mathrm{mL} / \mathrm{min}$ ). Paritaprevir, dasabuvir, and asunaprevir are contraindicated in patients with decompensated cirrhosis in whom the serum concentrations of these drugs are markedly increased. Absolute contraindications to the combination of PegIFN- $\alpha$ and ribavirin include uncontrolled depression or psychiatric illness, uncontrolled autoimmune diseases, transplantation of solid organs except the liver, untreated thyroid illness, pregnancy or unwillingness to comply with adequate contraception, severe concurrent medical illness such as poorly controlled hypertension, heart failure, significant coronary heart disease, poorly controlled diabetes mellitus, and chronic obstructive pulmonary disease, age $\leq 2$ years, and hypersensitivity to PegIFN- $\alpha$ or ribavirin.

\section{[Recommendations]}

1. All HCV-infected patients with no contraindication to treatment should be considered for treatment (A1).

2. Patients with advanced fibrosis $\geq F 3$ (including compensated and decompensated cirrhosis) should be given priority for treatment (A1).

3. Treatment should be prioritized in the pre- and post-liver transplant setting (A1)

4. Treatment should be prioritized for patients with severe extrahepatic manifestations, including HCV-related mixed cryoglobulinemia and glomerulonephritis (A1).

5. Treatment should be individualized taking into consideration the severity of liver disease, probability of treatment success, risks of severe adverse effects, accompanying diseases, and patients' willingness to undergo treatment (B1).

6. HCV treatment is not recommended in patients with limited life expectancy due to extrahepatic diseases (B1).

\section{DEFINITION OF TREATMENT RESPONSE}

An end-of-treatment response (ETR) is defined as undetectable HCV RNA at the end of treatment using a sensitive assay. An SVR is defined as undetectable HCV RNA by a sensitive assay at 12 or 24 weeks after completion of treatment. The concordance of SVR 12 and SVR 24 is $98 \%$ irrespective of treatment regimen. ${ }^{248}$ Viral breakthrough refers to the reappearance of HCV RNA during treat- 
The Korean Association for the Study of the Liver (KASL) KASL clinical practice guidelines: Management of Hepatitis C

Table 6. Definitions of virological responses during the combination therapy of PegIFN- $\alpha$ and ribavirin

\begin{tabular}{|c|c|c|}
\hline Virological response & Definition & Clinical implication \\
\hline Rapid virological response (RVR) & Undetectable HCV RNA ( $<50 \mathrm{IU} / \mathrm{mL})$ at week 4 of therapy & $\begin{array}{l}\text { May allow shortening of course } \\
\text { for genotypes } 2 \& 3 \text { and possibly } \\
\text { genotype } 1 \text { with low viral load }\end{array}$ \\
\hline Early virological response (EVR) & $\geq 2 \log$ reduction of HCV RNA level from baseline at week 12 of therapy & Negative predictor of SVR \\
\hline Complete EVR (c-EVR) & Undetectable HCV RNA at week 12 of therapy & \\
\hline Partial EVR (p-EVR) & EVR but detectable HCV RNA at week 12 of therapy & \\
\hline $\begin{array}{l}\text { Delayed virological response } \\
\text { (DVR) }\end{array}$ & $\begin{array}{l}\geq 2 \text { log reduction of HCV RNA level from baseline but detectable HCV } \\
\text { RNA at week } 12 \text { and undetectable HCV RNA at week } 24\end{array}$ & \\
\hline End of treatment response (ETR) & Undetectable HCV RNA at the end of 24 or 48 weeks of treatment & \\
\hline $\begin{array}{l}\text { Sustained virological response } \\
\text { (SVR) }\end{array}$ & Undetectable HCV RNA ( $<50 \mathrm{IU} / \mathrm{mL})$ at 12 or 24 weeks after treatment & $\begin{array}{l}\text { Best predictor of a long-term } \\
\text { response to treatment }\end{array}$ \\
\hline Null response (NR) & $<2$ log reduction of HCV RNA level from baseline at week 12 of therapy & \\
\hline Partial nonresponse & $\begin{array}{l}\geq 2 \text { log reduction of HCV RNA level from baseline but detectable HCV } \\
\text { RNA at week } 12 \text { and } 24\end{array}$ & \\
\hline Breakthrough & $\begin{array}{l}\text { Reappearance of HCV RNA in serum during treatment after virological } \\
\text { response }\end{array}$ & \\
\hline Relapse & Reappearance of HCV RNA after treatment is discontinued & \\
\hline
\end{tabular}

$\mathrm{HCV}$, hepatitis C virus.

ment after a virological response, and relapse is defined as the reappearance of HCV RNA after treatment has been completed.

The combination of PegIFN- $\alpha$ and ribavirin is associated with considerable cost and adverse effects. The likelihood of SVR increases as the time of HCV RNA disappearance is shorter. ${ }^{249} \mathrm{Re}$ sponse-guided therapy is a strategy to modify the duration of treatment based on the time of HCV RNA disappearance by measuring serum HCV RNA at weeks 4, 12, and 24 of treatment. However, treatment with DAA does not comply with this strategy.

A rapid virological response (RVR) is defined as undetectable HCV RNA using a sensitive assay with a lower limit of detection of $<50 \mathrm{IU} / \mathrm{mL}$ at week 4 of treatment. The SVR rate is expected to be $87.5-100 \%$ in HCV genotype 1 patients with a RVR and 33.3$63.8 \%$ in those without a RVR. ${ }^{250-252}$ The SVR rate is expected to be $85-86.5 \%$ in HCV genotypes 2 and 3 patients with a RVR and $54-58.3 \%$ in those without a RVR. ${ }^{251,253}$ An early virological response (EVR) is defined as undetectable HCV RNA using a sensitive assay with a lower limit of detection of $<50 \mathrm{IU} / \mathrm{mL}$ or $\mathrm{a} \geq 2$ log reduction in HCV RNA compared with the baseline level. The SVR rate is as low as $3 \%$ in HCV genotype 1 patients without an EVR. ${ }^{254-256}$ Therefore, medical cost and adverse effects can be reduced by discontinuing therapy in cases without an EVR. An EVR is classified as a complete EVR (CEVR), which is defined as unde- tectable HCV RNA, and a partial EVR (pEVR), which is defined as an EVR with detectable HCV RNA at week 12. A delayed virological response (DVR) is defined as a pEVR that results in undetectable HCV RNA at week $24{ }^{257,258}$ A null response is defined as a $<2$ log reduction in HCV RNA level from baseline at week 12 of therapy, whereas a partial nonresponse is defined as a $\geq 2$ log reduction in HCV RNA level from baseline but detectable HCV RNA at week 12 and 24 (Table 6).

\section{PREDICTORS OF TREATMENT RESPONSES}

HCV genotype $3{ }^{259}$ liver cirrhosis, ${ }^{259}$ previous treatment failure, ${ }^{259}$ and $\mathrm{RAV}^{233,260}$ are predictive factors for decreased SVR when treated with DAAs.

In cases of combination treatment of PegIFN- $\alpha$ and ribavirin, the strongest pretreatment predictors of an SVR include HCV genotype, ${ }^{254,261,262}$ degree of hepatic fibrosis, ${ }^{263}$ and IL28B genetic polymorphism. ${ }^{263,264}$ The SVR rates are $40-60 \%$ in HCV genotype 1 patients and $70-80 \%$ in HCV genotypes 2 and 3 patients. ${ }^{254,265}$ Patients with F0-F2 fibrosis have a 2.7-fold higher SVR rate than those with F3-F4 fibrosis. ${ }^{263}$ The SVR rates are 2.4-2.7-fold higher in patients with a viral load of $<400,000-800,000 \mathrm{IU} / \mathrm{mL}$ com- 
pared to those with a viral load of $>800,000 \mathrm{IU} / \mathrm{mL} .^{250,263,266}$ SVR rates are lower in older patients ( $>40$ years), ${ }^{254}$ African-Americans $^{267}$ body weight $>70 \mathrm{~kg}^{254,261}$ and insulin resistance. ${ }^{268,269}$ SVR rates vary depending on SNP of IL28B, in other words, a C or T allele at the rs12979869 locus. ${ }^{270}$ The SVR rates of HCV genotype 1 Caucasian patients are 69\%,33\%, and 27\% in CC homozygotes, CT heterozygotes, and TT homozygotes, respectively. The SVR rates of HCV genotype 1 African-American patients are 48\%, $15 \%$, and $13 \%{ }^{263,271}$ The SVR rates of HCV genotype 1 Korean patients are $73-88 \%$ in CC homozygotes and $0-40 \%$ in CT heterozygotes. ${ }^{272-274}$ The prevalence of the IL28B genetic polymorphism varies among ethnicities. CC homozygotes in Korea account for $88-89 \%,{ }^{272-274}$ compared to $17 \%$ in African-Americans, and $37 \%$ in Caucasians. ${ }^{263}$ Therefore, the usefulness of the IL28B genetic polymorphism as a predictive factor is limited as over $90 \%$ of the population of Korea are CC homozygotes.

During combination treatment with PegIFN- $\alpha$ and ribavirin, a $R V R$ is the strongest on-treatment predictor for an $S V R,{ }^{250-252}$ and an SVR rate increases ninefold with a RVR. ${ }^{263}$ Meanwhile, an EVR is a strong negative predictor for an SVR. Without EVR, the SVR rate is only $3 \% .{ }^{255}$ In addition, SVR rates increase when medication adherence is higher than $80 \%,{ }^{275}$ so assessment and maintenance of medication adherence can increase the SVR rate.

\section{NEW DRUGS, DIRECT-ACTING ANTIVIRALS (DAA)}

DAA acts at a specific step of the viral life cycle. DAAs include NS3/4A protease inhibitors (PI), NS5A inhibitors, and NS5B polymerase inhibitors. NS3/4A PI is a first-generation DAA and blocks the polyprotein processing essential for HCV replication. To date, available drugs are boceprevir, telaprevir, simeprevir, asunaprevir, and paritaprevir. NS5A inhibitors, such as daclatasvir, ledipasvir or ombitasvir, affect various HCV genotypes and show a synergistic effect in combination with other DAAs. The NS5B polymerase inhibitors are divided into nucleoside polymerase inhibitors (sofosbuvir) and non-nucleoside polymerase inhibitors (dasabuvir, beclabuvir). Because the basic characteristics of each DDA differ, selection and use of the appropriate drugs should take into consideration hepatic and renal function. DAA regimens may have a risk of interactions with other medications used by patients. Prior to starting treatment, patients should be evaluated for potential drug-drug interactions with selected DAAs. A more comprehensive list of drug-drug interactions is available at several websites, such as www.hep-druginteractions.org.

\section{[Recommendations]}

1. The characteristics of the DAAs should be understood, and the appropriate drugs selected taking into consideration hepatic and renal function (A1).

2. The potential for drug-drug interactions must be considered before and during treatment with DAAs. The full prescribing information must be consulted prior to use of DAAs due to the potential for drug-drug interactions (A1).

\section{Simeprevir}

Simeprevir is a HCV NS3/4A protease inhibitor.

\section{Dosage and administration}

One $150 \mathrm{mg}$ capsule is taken orally once daily with food.

\section{Pharmacokinetics}

Simeprevir primarily undergoes oxidative metabolism by the hepatic CYP3A system. Elimination of simeprevir occurs via biliary excretion. Renal clearance plays an insignificant role in its elimination. Therefore, no dose adjustment of simeprevir is needed in patients with mild, moderate or severe renal impairment. Compared to HCV-uninfected subjects with normal hepatic function, the mean steady-state AUC of simeprevir was higher in HCV-uninfected subjects with moderate hepatic impairment (Child-Pugh Class B) and severe hepatic impairment (Child-Pugh Class C). Simeprevir has not been extensively studied in such patients, but has been used in real-life settings.

\section{Drug-drug interactions}

Co-administration of simeprevir with substances that are moderate or strong inducers or inhibitors of CYP3A4 is not recommended as this may lead to significantly lower or higher exposure to simeprevir, respectively. A number of compounds are contraindicated in patients receiving simeprevir, including anticonvulsants (carbamazepine, phenobarbital, phenytoin), antibiotics (erythromycin, clarithromycin), antimycobacterials (rifampin, rifabutin, rifapentine), antifungals (itraconazole, ketoconazole, fluconazole, voriconazole), dexamethasone, cisapride, herbal products (milk thistle, St John's wort) and antiretroviral drugs (cobicistat-based regimens, efavirenz, etravirine, nevirapine, ritonavir). Simeprevir does not require dose changes in combination with the immuno- 
The Korean Association for the Study of the Liver (KASL) KASL clinical practice guidelines: Management of Hepatitis C

suppressants tacrolimus and sirolimus. In contrast, it is not recommended to co-administer simeprevir with cyclosporine, as this can result in significantly increased plasma concentrations of simeprevir. Dose adjustments are needed with some antiarrhythmics, warfarin, calcium-channel blockers, HMG Co-A reductase inhibitors and sedative/anxiolytics.

\section{Adverse reactions and safety}

Most common adverse reactions in patients receiving simeprevir, PegIFN- $\alpha$ and ribavirin were rash (including photosensitivity), pruritus and nausea. Transient hyperbilirubinemia was observed, but it was not associated with elevations in liver transaminase levels.

\section{Asunaprevir}

Asunaprevir is a HCV NS3/4A protease inhibitor.

\section{Dosage and administration}

One $100 \mathrm{mg}$ capsule is taken orally twice daily with or without food.

\section{Pharmacokinetics}

Asunaprevir undergoes oxidative metabolism primarily mediated by CYP3A. Following single-dose oral administration in healthy subjects, $84 \%$ of it was recovered in feces and less than $1 \%$ was recovered in the urine. No dosage adjustment is required for patients with mild and moderated renal impairment (eGFR 30-80 $\mathrm{mL} / \mathrm{min} / 1.73 \mathrm{~m}^{2}$ ). Dosage adjustment of asunaprevir to $100 \mathrm{mg}$ once daily is recommended for patients with severe renal impairment (eGFR $<30 \mathrm{~mL} / \mathrm{min} / 1.73 \mathrm{~m}^{2}$ ) not receiving hemodialysis. No dose adjustment of asunaprevir is required for patients with mild hepatic impairment. Asunaprevir is contraindicated for patients with moderate or severe hepatic impairment (Child Pugh B or C), because steady state exposure was markedly higher in those patients.

\section{Drug-drug interactions}

Co-administration asunaprevir with moderate or strong inducers or inhibitors of CYP3A is not recommended, as this may decrease or increase the plasma levels of asunaprevir. Furthermore, co-administration of asunaprevir with strong inhibitors of organic anion-transporting polypeptide (OATP)-mediated transport (e.g. rifampin, cyclosporine, sirolimus, gemfibrozil) is not recommended because this may increase the plasma concentration of asunaprevir and decrease its therapeutic effect.

\section{Adverse reactions and safety}

The most common adverse events were headache, fatigue, diarrhea, and nausea. ALT or AST elevations (3-4\%) were reported in clinical trials of asunaprevir-containing regimens.

\section{Daclatasvir}

Daclatasvir is a HCV NS5A inhibitor.

\section{Dosage and administration}

One $60 \mathrm{mg}$ tablet is taken orally once daily with or without food. If a reduced dose is needed, one $30 \mathrm{mg}$ tablet is taken once daily.

\section{Pharmacokinetics}

Daclatasvir is a substrate of CYP3A. Following single-dose oral administration in healthy subjects, $88 \%$ of the dose was recovered in feces and $6.6 \%$ was excreted in the urine. No dosage adjustment of daclatasvir is required for patients with any degree of renal impairment. Hepatic impairment does not have a clinically significant effect on the free drug concentrations of daclatasvir. Thus, no dosage adjustment of daclatasvir is required for patients with any degree of hepatic impairment.

\section{Drug-drug interactions}

Co-administration of daclatasvir with substances that are moderate or strong inducers or inhibitors of CYP3A4 is not recommended as this may lead to significantly lower or higher exposure of simeprevir, respectively.

\section{Adverse reactions and safety}

The most common adverse reactions observed with daclatasvir in combination with asunaprevir were headache, fatigue, diarrhea, nausea and elevation of ALT.

\section{Sofosbuvir}

Sofosbuvir is a HCV nucleotide analog NS5B polymerase inhibitor.

\section{Dosage and administration}

One $400 \mathrm{mg}$ tablet is taken orally once daily with or without food.

\section{Pharmacokinetics}

Sofosbuvir is extensively metabolized in the liver. The metabolic 
activation pathway involves sequential hydrolysis of the carboxyl ester moiety catalyzed by human cathepsin A (CatA) or carboxylesterase 1 (CES1) and phosphoramidate cleavage by histidine triad nucleotide-binding protein 1 (HINT1) followed by phosphorylation by the pyrimidine nucleotide biosynthesis pathway. Following a single $400 \mathrm{mg}$ oral dose, approximately $80 \%$ and $14 \%$ is respectively recovered in urine and feces. No dosage adjustment of sofosbuvir is required for patients with mild or moderate renal impairment (eGFR 30-80 mL/min $/ 1.73 \mathrm{~m}^{2}$ ). No dosage recommendation can be provided for patients with severe renal impairment (eGFR $<30 \mathrm{~mL} / \mathrm{min} / 1.73 \mathrm{~m}^{2}$ ) or end-stage renal disease. No dosage adjustment of sofosbuvir is required for patients with hepatic impairment.

\section{Drug-drug interactions}

Sofosbuvir is a substrate of the drug transporter P-gp. Drugs that are P-gp inducers in the intestine (e.g., rifampin, carbamazepine, phenytoin, St. John's wort) may decrease sofosbuvir plasma concentration, leading to a reduced therapeutic effect, and thus concomitant use with sofosbuvir is not recommended. In addition, since serious symptomatic bradycardia may occur, co-administration of amiodarone with sofosbuvir in combination with another DAA, such as daclatasvir, simeprevir or ledipasvir, is contraindicated.

\section{Adverse reactions and safety}

The most common adverse reactions observed with sofosbuvir in combination with ribavirin were fatigue and headache. The most common adverse events observed with sofosbuvir, PegIFN- $\alpha$ and ribavirin were fatigue, headache, nausea, insomnia and anemia.

\section{Ledipasvir/Sofosbuvir}

Ledipasvir/sofosbuvir is a fixed-dose combination of ledipasvir, a HCV NS5A inhibitor, and sofosbuvir, an HCV nucleotide analog NS5B polymerase inhibitor.

\section{Dosage and administration}

One tablet (90 mg of ledipasvir and $400 \mathrm{mg}$ of sofosbuvir) is taken orally once daily with or without food.

\section{Pharmacokinetics}

In vitro, no detectable metabolism of ledipasvir by human $\mathrm{CY}$ P1A2, CYP2C8, CYP2C9, CYP2C19, CYP2D6, and CYP3A4 was observed. Evidence of slow oxidative metabolism via an unknown mechanism has been reported. Biliary excretion of unchanged le- dipasvir is a major route of elimination, with renal excretion being a minor pathway. No dose adjustment is required for patients with mild or moderate renal impairment (eGFR $30-80 \mathrm{~mL} /$ $\min / 1.73 \mathrm{~m}^{2}$ ), but no dose recommendation can currently be provided for patients with severe renal impairment (eGFR $<30 \mathrm{~mL}$ / $\mathrm{min} / 1.73 \mathrm{~m}^{2}$ ) or with end-stage renal disease (ESRD). No dosage adjustment of ledipasvir/sofosbuvir is required for patients with hepatic impairment.

\section{Drug-drug interactions}

Ledipasvir is an inhibitor of the drug transporter P-gp and may increase intestinal absorption of co-administered substrates of these transporters. Thus, caution is needed with P-gp substrates such as digoxin and dabigatran such as digoxin and dabigatran, but also potentially with other drugs that are, in part, transported by these proteins (e.g. aliskiren, amlodipine, buprenorphine, carvedilol, cyclosporine). Co-administration of amiodarone (and possibly dronedarone) with sofosbuvir/ledipasvir is contraindicated due to a serious risk of symptomatic bradycardia. Co-administration of sofosbuvir/ledipasvir with rosuvastatin may significantly increase the concentration of rosuvastatin, which is associated with an increased risk of myopathy, including rhabdomyolysis. Coadministration of sofosbuvir/ledipasvir with rosuvastatin is not recommended. The solubility of ledipasvir decreases with increasing pH. Drugs that increase gastric pH (e.g., antacids, H2-receptor antagonists, or proton pump inhibitors) are expected to decrease the concentration of ledipasvir. Because sofosbuvir/ledipasvir increases the tenofovir concentration when a pharmacokinetic enhancer (ritonavir or cobicistat) is present in an antiretroviral regimen, these combinations should be used with caution, with frequent renal monitoring if other alternatives are not available.

\section{Adverse reactions and safety}

The most common adverse reactions observed with ledipasvir/ sofosbuvir were fatigue, headache, nausea, diarrhea and insomnia.

\section{Ombitasvir/paritaprevir/ritonavir and dasabuvir}

Ombitasvir/paritaprevir/ritonavir and dasabuvir (Opr+D) includes ombitasvir, a HCV NS5A inhibitor, paritaprevir, a HCV NS3/4A protease inhibitor, ritonavir, a CYP3A inhibitor and dasabuvir, a HCV non-nucleoside NS5B palm polymerase inhibitor.

\section{Dosage and administration}

Two ombitasvir, paritaprevir, ritonavir 12.5/75/50 mg tablets 
The Korean Association for the Study of the Liver (KASL) KASL clinical practice guidelines: Management of Hepatitis C

once daily (in the morning) and one dasabuvir $250 \mathrm{mg}$ tablet twice daily (morning and evening) are taken orally with a meal.

\section{Pharmacokinetics}

Ombitasvir is predominantly metabolized by amide hydrolysis followed by oxidative metabolism. Paritaprevir is predominantly metabolized by CYP3A4 and dasabuvir is predominantly metabolized by CYP2C8. Paritaprevir and ombitasvir are excreted predominantly into the feces. Dasabuvir is metabolized in the liver, and its predominant metabolite is cleared mainly via biliary excretion and fecal elimination with minimal renal clearance. No dosage adjustment is required in patients with mild, moderate or severe renal impairment. It has not been studied in patients on dialysis. No dosage adjustment is required in patients with mild hepatic impairment (Child-Pugh A). However, it is not recommended in patients with moderate or severe hepatic impairment (Child-Pugh B or C).

\section{Drug-drug interactions}

Co-administration of Opr+D can alter the plasma concentrations of some drugs and some drugs may alter the plasma concentrations of Opr+D. The potential for drug interactions must be considered before and during treatment. Co-administration of Opr+D with drugs that are highly dependent on CYP3A for clearance; moderate or strong inducers of CYP3A and strong inducers of CYP2C8; and strong inhibitors of CYP2C8 is not recommended.

\section{Adverse reactions and safety}

The most commonly reported adverse reactions were nausea, pruritus, and insomnia.

\section{TREATMENT OF CHRONIC HEPATITIS C}

Recently, there has been marked improvement in the treatment of chronic hepatitis $C$, which is sufficient to be called a paradigm shift. The effectiveness of the combined administration of PegIFN- $\alpha$ and ribavirin, which was the previous standard treatment, has been decreased due to its relatively low treatment effects and severe side effects. Furthermore, the combined administration of PeglFN- $\alpha /$ ribavirin and the first-generation NS3/4A PI (boceprevir, telaprevir) was also limited due to high cost, drug resistance and exacerbated side effects.

DAA acts on a specific part of the HCV non-structural (nonstructural, NS) protein, blocking HCV replication and it is classi- fied as a NS3/4A PI, NS5A inhibitor and NS5B nucleos(t)ide/nonnucleoside polymerase inhibitor based on its mechanism and target. Since drugs show advantages and disadvantages, use of a combination of two or more DAAs with different targets or addition of ribavirin in a difficult-to-treat patient shows an SVR rate of $\sim 90 \%$ after 12 weeks of treatment or expanded treatment for 24 weeks.

In Korea, boceprevir was approved in May 2014 by the Ministry of Food and Drug Safety as a DAA for the treatment of chronic hepatitis C. Daclatasvir (NS5A inhibitor), asunaprevir (secondgeneration PI), sofosbuvir (NS5B nucleotide polymerase inhibitor), and ledipasvir (NS5A inhibitor)/sofosbuvir, which administered as a fixed-dose in a single tablet, have been approved up to November 2015. Various types of DAA are awaiting approval which will improve the treatment of chronic hepatitis C. However, high costs, drug resistance and interactions between various drugs remain to be overcome. Initially, the combined administration of PegIFN- $\alpha$ and ribavirin showed good treatment effects in some genotypes, is less expensive than DAA and is useful in situations where DAAs cannot be used. Hopefully, DAAs with excellent cost-effectiveness, shorter treatment duration, effective in broad spectrum of patients, less side effects and minimal drug-drug interactions will be available in the near future.

\section{TREATMENT OF HCV GENOTYPE 1 INFECTION}

\section{Initial treatment}

The SVR rate was 99\% in 865 treatment-naïve patients with HCV genotype 1 infection who received ledipasvir/sofosbuvir for 12 weeks. There were no additional benefits when ribavirin was added or the duration of treatment was extended to 24 weeks. ${ }^{276}$ No significant difference in SVR rate was found according to age, baseline HCV RNA level, presence of cirrhosis, IL28B genotype, or HCV gene sub-genotype (1a or 1b). RAVs of ledipasvir at baseline were detected in $16 \%$ of the patients, but had no significant effect on the treatment result. ${ }^{277}$ In a study involving patients with baseline HCV RNA levels of $<6,000,000 \mathrm{IU} / \mathrm{mL}$, the SVR rates were $97 \%$ in those who received 8 weeks of combination therapy with ledipasvir/sofosbuvir plus ribavirin and $96 \%$ in those who received 12 weeks of ledipasvir/sofosbuvir, suggesting a shortened treatment duration in some patients. ${ }^{278}$ In an integrated analysis of 513 patients with genotype 1 compensated liver cirrhosis who 
received a combination of ledipasvir/sofosbuvir plus ribavirin or ledipasvir/sofosbuvir, the SVR rates were $95 \%$ in those who received the therapy for 12 weeks and $98 \%$ in those who received it for 24 weeks, showing no additional benefit in previously untreated patients with liver cirrhosis of ribavirin addition or extension of treatment duration to 24 weeks. ${ }^{279}$

In treatment-naïve patients without cirrhosis treated with ombitasvir/paritaprevir/ritonavir and dasabuvir (Opr+D) together with ribavirin for 12 weeks, the SVR rates were 95\% (307/322) in genotype $1 \mathrm{a}$ and $98 \%$ (148/151) in genotype $1 \mathrm{~b}$ patients. ${ }^{280}$ To evaluate the importance of ribavirin administration with $\mathrm{Opr}+\mathrm{D}$, phase 3 trials were conducted in treatment-naïve, non-cirrhotic patients with genotype $1 \mathrm{a}$ and $1 \mathrm{~b} .{ }^{281}$ Patients with HCV genotype $1 \mathrm{~b}$ were randomly assigned to receive $\mathrm{Opr}+\mathrm{D}$ alone $(\mathrm{n}=209)$ or with ribavirin $(n=210)$ for 12 weeks. Only three of 419 patients in the trial failed treatment; the SVR rate was $99 \%$ in both groups. On the other hand, of 205 patients with HCV genotype 1a randomly assigned to receive Opr+D alone for 12 weeks, $185(90 \%)$ achieved SVR; this rate was significantly lower than that observed in patients treated with Opr+D plus ribavirin (97\%), emphasizing the importance of ribavirin co-administration when Opr+D is prescribed to patients with HCV genotype 1a. In treatment-naïve patients with compensated cirrhosis, SVR rates were $92 \%$ and $93 \%$ in patients infected with genotype 1a and $100 \%$ in patients infected with genotype $1 \mathrm{~b}$ when treated with $0 \mathrm{pr}+\mathrm{D}$ plus ribavirin for 12 and 24 weeks, respectively. ${ }^{282}$ Recently, the Opr+D regimen, without ribavirin, for 12 weeks achieved a 100\% SVR in 60 genotype $1 \mathrm{~b}$ patients with compensated cirrhosis, including treatment-experienced patients. ${ }^{283}$ On the basis of this study, treating patients infected with genotype $1 \mathrm{~b}$ with Opr+D without ribavirin for 12 weeks is recommended, irrespective of the presence of cirrhosis or prior treatment experience.

In treatment-naïve HCV genotype $1 \mathrm{~b}$ patients treated with daclatasvir and asunaprevir for 24 weeks, the SVR rate was $90 \% .{ }^{233}$ Among Korean patients, 95\% (20/21) achieved a SVR. ${ }^{284}$ There were no differences in SVR rates based on sex, age, race, IL28B genotype, or presence of cirrhosis. However, multivariate regression analysis of baseline factors identified the presence of NS5A RAVs L31F/I/M/V and/or Y93H as negative predictors of SVR. Also, pooled data from five clinical studies of DCV and ASV in HCV genotype $1 \mathrm{~b}$ patients show that the presence of the NS5A RAVs L31F//M/M and/or Y93H at baseline was associated with a reduced SVR (range: 36.9-41.9\%), while SVR rates in the absence of these RAVs were high (range: 88.0-93.9\%). ${ }^{163}$ Pretreatment NS5A RAVs L31F//M/V and/or Y93H were present in 12.6-14.4\% of HCV genotype $1 \mathrm{~b}$ patients. Therefore, this combination is not recommended in patients with detectable NS5A RAVs L31F/I/M/V and/or $\mathrm{Y} 93 \mathrm{H}$ at baseline.

In a study carried of combination treatment of sofosbuvir and simeprevir for 12 weeks or 24 weeks, the SVR rate of all patients was $92 \%$ (154/167 patients), and there was no difference in SVR rate according to treatment duration (12 or 24 weeks), addition of ribavirin or fibrotic stage. However, the treatment effect of genotype 1a patients with Q80K RAV was lower in comparison to patients with no RAV (88\% vs. $94 \%$ ). ${ }^{285}$ In a recent phase 3 study, the SVR rate of genotype 1 patients without liver cirrhosis was 97\% (150/155), and there was no difference according to the subgenotype. ${ }^{286}$ In a study carried out on 103 patients with liver cirrhosis, the SVR rate was $84 \%(86 / 103)$, and there was no difference according to sub-genotype. ${ }^{287}$ In a real-life study, the SVR rate of 276 (including 132 treatment-naïve) patients was 82\%, and among them, the SVR rate of those with liver cirrhosis was $\sim 10 \%$ lower ( $88 \%$ vs. $75 \%$ ). ${ }^{288}$

In an open-label study of 111 treatment-naïve patients receiving treatment with daclatasvir and sofosbuvir with or without ribavirin, SVR was achieved in $98 \%$ irrespective of treatment duration. ${ }^{234}$

Treatment with a combination of sofosbuvir and PegIFN- $\alpha$ plus ribavirin for 12 weeks resulted in an 89\% SVR rate among 291 previously untreated patients with genotype $1 .{ }^{259}$ In a study that compared combination therapy with sofosbuvir and PegIFN- $\alpha$ plus ribavirin using treatment durations of 12 and 24 weeks, the SVR rate in both groups was $89 \%$. Treatment for 24 weeks had no additional benefit compared with treatment for 2 weeks. ${ }^{289}$

When combination treatment of simeprevir with PegIFN- $\alpha$ and ribavirin was carried out for 12 weeks, followed by combination treatment of PegIFN- $\alpha$ and ribavirin for 12 or 36 weeks according to the therapeutic response (response-guided therapy) in a study of 305 (including 60 Korean) patients, the SVR rate was $90 \%$. In another study of 264 patients, the SVR rate was $80 \%$ (1b: $90 \%$ [105/117], 1a: 71\% [105/147]) using the same regimen. Among genotype 1a patients, the SVR rate of those with Q80K RAV was $52 \%(31 / 60)$, and that of patients with no RAV was $85 \%(73 / 86)$, which was similar to the result of genotype $1 \mathrm{~b}$ patients. However, the SVR rate of 31 patients with liver cirrhosis was $58 \%$ $(18 / 31){ }^{290,291}$

SVR rates in Europe and the US were reported as $40-50 \%$ when HCV genotype 1 patients were treated with PegIFN- $\alpha$ and ribavirin. ${ }^{292}$ SVR rates in South Korea were reported higher; 53.6$69.5 \%{ }^{293-297}$ and $62.7 \%$ in a pooled analysis of 10 studies. ${ }^{298}$ This 
The Korean Association for the Study of the Liver (KASL) KASL clinical practice guidelines: Management of Hepatitis C

is related to the higher frequency of favorable IL28B genotypes in Koreans than in Caucasians or African-Americans. ${ }^{83}$ Patients with genotype 1 who achieve a RVR and had a low baseline viral load $(<400,000 \mathrm{IU} / \mathrm{mL})$ may have their duration of therapy shortened to 24 weeks if there are no negative predictors of response, such as advanced liver fibrosis, cirrhosis, obesity, or insulin resistance. ${ }^{249}$ Treatment should be stopped in patients who do not achieve an EVR, as the SVR rate in these patients using the standard treatment duration is $<3 \%$, and in patients with $\mathrm{PEVR}$ and detectable HCV RNA at week 24 , as the SVR rate is $2-4 \%$. ${ }^{254,299}$

\section{Retreatment of treatment-experienced patients}

Few Korean HCV patients have experience of PI, except for those who participated in clinical trials. Therefore, in the current guidelines, "treatment-experienced patients" refer to the patients treated with conventional interferon or PegIFN- $\alpha$ in combination with ribavirin, not including DAAs. These include patients who previously received treatment but failed to achieve an SVR due to various factors (e.g., non-response or partial response to treatment, relapse, virological breakthrough, and drug adverse events or low compliance). In addition, in the case of genotype 1 with an undistinguishable sub-genotype, treatment is to be given in accordance with genotype 1a and drugs that can be used unrelated to sub-genotypes are recommended.

of 440 patients treated with ledipasvir/sofosbuvir for 12 weeks, $44 \%$ were un-responsive to prior treatment and $53 \%$ had prior $\mathrm{PI}$ treatment failure, resulting in a 94\% SVR rate, showing no significant difference between treatment with a combination of ledipasvir/sofosbuvir plus ribavirin for 12 weeks (SVR rate 96\%) and treatment with ledipasvir/sofosbuvir for 24 weeks (SVR rate $99 \%) .{ }^{300}$ NS5A RAVs at baseline were detected in $14 \%$ of the patients, of whom $89 \%$ showed a treatment response. Baseline viral load, age, presence of liver cirrhosis, IL28B genotype, and subgenotype had no significant effect on treatment response. In a Korean study of 93 patients (46 treatment-naïve patients and 47 patients with prior treatment failure), including previously treated patients, the SVR rates were 99\% (92/93) when they were given ledipasvir/sofosbuvir for 12 weeks and 100\% (17/17) among patients with cirrhosis who received the same regimen. In that study, NS5A RAVs at baseline were detected in $22 \%$ of the patients, of whom 95\% showed a treatment response. ${ }^{301}$ In addition, in another study, treatment with ledipasvir/sofosbuvir for 12 weeks resulted in a treatment response in all 341 previously treated patients, including patients with cirrhosis. ${ }^{302}$ In an integrated analysis of patients with compensated cirrhosis, the SVR rates were 90\% (162/171) when ledipasvir/sofosbuvir was administered for 12 weeks, 96\% (174/181) when a combination of ledipasvir/sofosbuvir plus ribavirin was administered for 12 weeks, $98 \%$ (98/100) when ledipasvir/sofosbuvir was administered for 24 weeks, and 100\% (22/22) when ledipasvir/sofosbuvir plus ribavirin was administered for 24 weeks. Therefore, addition of ribavirin or extension of treatment duration was more effective. ${ }^{279}$ Treatment with a combination of ledipasvir/sofosbuvir plus ribavirin for 12 weeks resulted in a 96\% SVR rate among 155 patients with cirrhosis who had treatment failure after the treatment with combination of PegIFN- $\alpha$ and ribavirin, followed by retreatment failure, including PI. This is similar to the results of treatment with ledipasvir/sofosbuvir for 24 weeks, which resulted in a 97\% SVR rate. ${ }^{303}$

The combination of ombitasvir/paritaprevir/ritonavir and dasabuvir (Opr+D) plus ribavirin for 12 weeks was evaluated in 297 non-cirrhotic patients in whom previous PegIFN- $\alpha$ and ribavirin therapy failed. ${ }^{304}$ The SVR rate was $96 \%$, and did not differ according to sub-genotypes, or types of treatment failure. To evaluate the importance of ribavirin in non-cirrhotic, treatment-experienced patients with HCV genotype 1b infection, 179 patients in whom previous therapy with PegIFN- $\alpha$ and ribavirin failed were treated with Opr+D with or without ribavirin for 12 weeks. ${ }^{305}$ SVR rates were high in both arms: 100\% (91/91) in the ribavirin-free arm and $96.6 \%$ (85/88) in the ribavirin-containing arm, supporting the recommendation that Opr+D may be used without ribavirin for patients with HCV genotype $1 b$ infection. Among treatment-experienced patients with compensated cirrhosis, SVR rates were $86 \%$ and $93 \%$ in those infected with genotype 1a and $98 \%$ and $100 \%$ in patients infected with genotype $1 \mathrm{~b}$ when treated with Opr+D plus ribavirin for 12 and 24 weeks, respectively. ${ }^{282}$ Recently, treatment using the Opr+D regimen, without ribavirin, for 12 weeks achieved a 100\% SVR in 60 genotype 1b patients with compensated cirrhosis, including treatment-experienced patients. ${ }^{283}$

In treatment-experienced HCV sub-genotype $1 \mathrm{~b}$ patients treated with daclatasvir and asunaprevir for 24 weeks, the SVR rates were $82 \%$ in non-responder patients and $82 \%$ in interferon-ineligible/intolerant patients. ${ }^{233}$ The SVR rate was higher in treatmentnaïve patients versus experienced patients. In 44 Korean treatment-experienced HCV genotype $1 \mathrm{~b}$ patients, an SVR was achieved by $70 \%$ of non-responder patients and $86 \%$ of interferon-ineligible/intolerant patients. ${ }^{284}$ In an open-label, phase 3, Japanese study, an SVR was achieved in $87 \%$ of patients who were ineligible or intolerant to interferon-based therapy and in $81 \%$ of 
Table 7. Treatment of HCV genotype 1 infection in chronic hepatitis or compensated cirrhosis

\begin{tabular}{|c|c|c|c|c|c|}
\hline & & \multicolumn{2}{|c|}{ Genotype 1b } & \multicolumn{2}{|c|}{ Genotype 1a } \\
\hline & & $\begin{array}{l}\text { Chronic } \\
\text { hepatitis }\end{array}$ & $\begin{array}{l}\text { Compensated } \\
\text { cirrhosis }\end{array}$ & $\begin{array}{l}\text { Chronic } \\
\text { hepatitis }\end{array}$ & $\begin{array}{l}\text { Compensated } \\
\text { cirrhosis }\end{array}$ \\
\hline \multirow[t]{7}{*}{ Treatment naïve } & Ledipasvir/sofosbuvir & \multicolumn{2}{|c|}{$12 \mathrm{wk}$} & \multicolumn{2}{|c|}{$12 \mathrm{wk}$} \\
\hline & $\mathrm{OPr}+\mathrm{D}$ & \multicolumn{2}{|c|}{$12 w k$} & $12 w k+R$ & $24 w k+R$ \\
\hline & Daclatasvir+Asunaprvir & \multicolumn{2}{|c|}{$24 w k$} & & \\
\hline & Sofosbuvir+Simeprevir & $12 w k$ & $12 w k+R / 24 w k$ & $12 w k$ & $12 w k+R / 24 w k$ \\
\hline & Daclatasvir+Sofosbuvir & $12 w k$ & $12 w k+R / 24 w k$ & $12 w k$ & $12 w k+R / 24 w k$ \\
\hline & Sofosbuvir+PR & \multicolumn{2}{|c|}{12 wk } & \multicolumn{2}{|c|}{$12 w k$} \\
\hline & $P R$ & \multicolumn{2}{|c|}{$24-48 w k$} & \multicolumn{2}{|c|}{$24-48 w k$} \\
\hline \multirow[t]{5}{*}{ PR experienced } & Ledipasvir/sofosbuvir & $12 w k$ & $12 w k+R / 24 w k$ & 12 wk & $12 w k+R / 24 w k$ \\
\hline & $\mathrm{OPr}+\mathrm{D}$ & \multicolumn{2}{|c|}{$12 w k$} & $12 w k+R$ & $24 w k+R$ \\
\hline & Daclatasvir+Asunaprvir & \multicolumn{2}{|c|}{$24 w k$} & & \\
\hline & Sofosbuvir+Simeprevir & $12 w k$ & $12 w k+R / 24 w k$ & $12 w k$ & $12 w k+R / 24 w k$ \\
\hline & Daclatasvir+Sofosbuvir & $12 w k$ & $12 w k+R / 24 w k$ & $12 w k$ & $12 w k+R / 24 w k$ \\
\hline
\end{tabular}

$\mathrm{OPr}+\mathrm{D}$, ombitasvir/paritaprevir/ritonavir+dasabuvir; R, weight-based ribavirin; PR, pegylated interferon- $\alpha$ +ribavirin therapy.

patients who had not responded to treatment previously. ${ }^{306}$ There were no differences in SVR rates based on sex, age, race, IL28B genotype, or presence of cirrhosis.

The SVR rate of patients who showed no response to the previous combination treatment of PegIFN- $\alpha$ and ribavirin was 91$95 \%$, similar to that of patients without experience of combination treatment with sofosbuvir and simeprevir. However, the treatment-experienced patients with liver cirrhosis showed a lower SVR rate than treatment-naïve patients (79\% vs. $88 \%$ ). ${ }^{285-287}$

In an open-label study, 41 patients for whom treatment including PegIFN- $\alpha$-based therapy and a first-generation PI had failed were treated for 24 weeks with daclatasvir and sofosbuvir without ribavirin $(n=20)$ and with ribavirin $(n=21) .{ }^{234}$ The SVR rate was $98 \%$. In a real-life French cohort, 409 HCV genotype 1 patients (307 treatment-experienced including a first-generation PIs, 319 patients with cirrhosis) were treated with daclatasvir and sofosbuvir with or without ribavirin for 12 or 24 weeks. ${ }^{307}$ Overall, SVR rates for daclatasvir and sofosbuvir without ribavirin were $85 \%$ after 12 weeks of treatment and $93 \%$ following 24 weeks of treatment. The SVR rates for daclatasvir and sofosbuvir with ribavirin were $100 \%$ and $98 \%$ after 12 and 24 weeks, respectively.

When combination treatment of simeprevir with PegIFN- $\alpha$ and ribavirin was carried out for 12 weeks in relapsed patients, followed by combination treatment of PegIFN- $\alpha$ and ribavirin for 12 or 36 weeks according to the therapeutic responses (responseguided therapy), the SVR rate was 79\% (206/260), and among them, genotype $1 \mathrm{~b}$ showed $86 \%$ and 1 a showed $70 \% .{ }^{260}$ The SVR rate of patients who showed a partial response to previous treatments was $70 \%(101 / 145)$, and that of patients who showed no response to previous treatments (non-responders) was $44 \%$ (102/234), indicating that this regimen showed a very low therapeutic effect in patients who did not respond to previous treatments. $^{308}$

\section{[Recommendations] (Table 7)}

\section{Initial treatment of HCV genotype 1 infection}

1. Patients infected with HCV genotype 1 with or without cirrhosis should be treated with daily ledipasvir $(90 \mathrm{mg}) / \mathrm{sofos}-$ buvir (400 mg) for 12 weeks, regardless of sub-genotype (A1).

2. Patients infected with HCV genotype $1 \mathrm{~b}$ with or without cirrhosis should be treated with daily ombitasvir $(25 \mathrm{mg}) /$ paritaprevir (150 mg)/ritonavir (100 mg) plus dasabuvir (500 $\mathrm{mg}$ ) for 12 weeks (A1).

3. Patients infected with HCV genotype 1a without cirrhosis should be treated with daily ombitasvir $(25 \mathrm{mg}) /$ paritaprevir (150 mg)/ritonavir (100 mg) plus dasabuvir $(500 \mathrm{mg})$ and weight-based ribavirin $(1,200 \mathrm{mg}$ in patients $\geq 75 \mathrm{~kg}, 1,000$ $\mathrm{mg}$ in patients $<75 \mathrm{~kg}$ ) for 12 weeks (A1). Duration of therapy should be extended to 24 weeks in patients with liver cirrhosis (A1).

4. Patients infected with HCV genotype $1 \mathrm{~b}$ with or without cir- 
The Korean Association for the Study of the Liver (KASL) KASL clinical practice guidelines: Management of Hepatitis C

rhosis should be treated with daily daclatasvir $(60 \mathrm{mg})$ and asunaprevir $(200 \mathrm{mg})$ for 24 weeks. Patients infected with HCV genotype $1 \mathrm{~b}$ in whom treatment with daclatasvir and asunaprevir is considered must be tested for NS5A RAVs L31F/I/M/V and/or Y93H prior to treatment. Patients with NS5A RAVs L31F///M/V and/or Y93H should be treated with an alternative regimen (A1).

5. Patients infected with HCV genotype 1 without cirrhosis should be treated with daily sofosbuvir (400 mg) and simeprevir (150 mg) for 12 weeks, regardless of sub-genotype (A1). Addition of daily weight-based ribavirin (1,200 $\mathrm{mg}$ in patients $\geq 75 \mathrm{~kg}, 1,000 \mathrm{mg}$ in patients $<75 \mathrm{~kg}$ ) is recommended in patients with cirrhosis (B1). Extending treatment to 24 weeks is recommended for patients with cirrhosis in whom use of ribavirin is contraindicated (B1).

6. Patients infected with HCV genotype 1 without cirrhosis should be treated with daily daclatasvir $(60 \mathrm{mg})$ and sofosbuvir (400 mg) for 12 weeks, regardless of sub-genotype (A1). Adding daily weight-based ribavirin $(1,200 \mathrm{mg}$ in patients $\geq 75 \mathrm{~kg}, 1,000 \mathrm{mg}$ in patients $<75 \mathrm{~kg}$ ) is recommended for patients with cirrhosis (B1). Extending treatment to 24 weeks is recommended for patients with cirrhosis in whom use of ribavirin is contraindicated (B1).

7. Patients infected with HCV genotype 1 with or without cirrhosis should be treated with daily sofosbuvir $(400 \mathrm{mg})$ and weight-based ribavirin $(1,200 \mathrm{mg}$ in patients $\geq 75 \mathrm{~kg}, 1,000 \mathrm{mg}$ in patients $<75 \mathrm{~kg}$ ) plus weekly PegIFN- $\alpha$ for 12 weeks (A2).

8. Treatment with one of two PegIFN- $\alpha$ molecules in combination with ribavirin should be planned for 48 weeks (A2). PegIFN- $\alpha 2$ a should be injected $180 \mu \mathrm{g}$ subcutaneous once a week, regardless of patient body weight with ribavirin using doses of $1,000 \mathrm{mg} / \mathrm{d}$ for those $\leq 75 \mathrm{~kg}$ in weight and $1,200 \mathrm{mg} /$ day for those $>75 \mathrm{~kg}$. PeglFN- $\alpha 2 \mathrm{~b}$ is to be injected $1.5 \mu \mathrm{g} / \mathrm{kg} /$ week with ribavirin using doses of $800 \mathrm{mg}$ for those $<65 \mathrm{~kg}$ in weight, 1,000 mg for $65-85 \mathrm{~kg}, 1,200 \mathrm{mg}$ for $85-105 \mathrm{~kg}$, and 1,400 mg for $>105 \mathrm{~kg}$. In patients with a RVR and low baseline HCV viral load $(<400,000 \mathrm{IU} / \mathrm{mL})$, and without any negative predictors of SVR (advanced liver fibrosis, cirrhosis, obesity or insulin resistance), shortening of treatment duration to 24 weeks can be considered (B1). Treatment should be stopped in patients who fail to achieve an EVR (A1). Patients who achieve a cEVR can be treated for 48 weeks (A1). Patients with a pEVR should be re-tested at week 24; if HCV RNA remains positive, treatment should be stopped (A1).
Retreatment of treatment-experienced patients with HCV genotype 1

(In this guideline, "treatment-experienced patients" refer to patients who have been treated with conventional interferon or PegIFN- $\alpha$ and ribavirin, not including DAAs. These include patients who previously underwent treatment but failed to reach SVR due to various factors (e.g., non-response or partial response to treatment, relapse, virological breakthrough, and drug adverse events or low compliance). In addition, in the case of genotype 1 with an undistinguishable sub-genotype, treatment is to be given in accordance with genotype 1a and drugs that can be used unrelated to sub-genotype are recommended.)

1. Treatment-experienced patients infected with HCV genotype 1 without cirrhosis should be treated with daily ledipasvir $(90 \mathrm{mg}) / \mathrm{sofosbuvir}(400 \mathrm{mg}$ ) for 12 weeks, regardless of sub-genotype (A1). Duration of therapy should be extended to 24 weeks in patients with liver cirrhosis (A1). Addition of daily weight-based ribavirin $(1,200 \mathrm{mg}$ in patients $\geq 75 \mathrm{~kg}, 1,000 \mathrm{mg}$ in patients $<75 \mathrm{~kg}$ ) without extending treatment duration can also be recommended (B1).

2. Treatment-experienced patients infected with HCV genotype $1 \mathrm{~b}$ with or without cirrhosis should be treated with daily ombitasvir (25 mg)/paritaprevir (150 mg)/ritonavir (100 $\mathrm{mg}$ ) plus dasabuvir (500 mg) for 12 weeks (A1).

3. Treatment-experienced patients infected with HCV genotype 1a without cirrhosis should be treated with daily ombitasvir (25 mg)/paritaprevir (150 mg)/ritonavir (100 mg) plus dasabuvir (500 mg) and weight-based ribavirin (1,200 $\mathrm{mg}$ in patients $\geq 75 \mathrm{~kg}, 1,000 \mathrm{mg}$ in patients $<75 \mathrm{~kg}$ ) for 12 weeks (A1). Duration of therapy should be extended to 24 weeks in patients with cirrhosis (A1).

4. Treatment-experienced patients infected with HCV genotype $1 \mathrm{~b}$ with or without cirrhosis should be treated with daily daclatasvir $(60 \mathrm{mg}$ ) and asunaprevir $(200 \mathrm{mg})$ for 24 weeks. Patients infected with HCV genotype $1 \mathrm{~b}$ in whom treatment with daclatasvir and asunaprevir is considered must be tested for the NS5A RAVs L31F/I/M/V and/or Y93H prior to treatment. Patients with the NS5A RAVs L31F/I/M/V and/or Y93H should be treated with an alternative regimen (A1).

5. Treatment-experienced patients infected with HCV genotype 1 without cirrhosis should be treated with daily sofosbuvir (400 mg) and simeprevir (150 mg) for 12 weeks, regardless of sub-genotype (A1). Addition of daily weightbased ribavirin $(1,200 \mathrm{mg}$ in patients $\geq 75 \mathrm{~kg}, 1,000 \mathrm{mg}$ in patients $<75 \mathrm{~kg}$ ) for 12 weeks is recommended (B1). Ex- 
tending treatment to 24 weeks is recommended for patients with cirrhosis in whom use of ribavirin is contraindicated (B1).

6. Treatment-experienced patients infected with HCV genotype 1 without cirrhosis can be treated with daily daclatasvir (60 mg) and sofosbuvir (400 mg) for 12 weeks, regardless of sub-genotype (B1). Addition of daily weight-based ribavirin $(1,200 \mathrm{mg}$ in patients $\geq 75 \mathrm{~kg}, 1,000 \mathrm{mg}$ in patients $<75 \mathrm{~kg})$ is recommended for patient with cirrhosis (B1). Extending treatment to 24 weeks is recommended for patients with cirrhosis in whom use of ribavirin is contraindicated (B1).

\section{TREATMENT OF HCV GENOTYPE 2 INFECTION}

\section{Initial treatment}

In the FISSION study in which treatment-naïve chronic HCV genotype 2 patients were treated with sofosbuvir and weightbased ribavirin for 12 weeks, the SVR rate was 97\% (68/70). ${ }^{259}$ This was significantly higher than that of 24 weeks of treatment using PegIFN- $\alpha$ and ribavirin, which showed an SVR rate of $78 \%$ $(52 / 67) .{ }^{259}$ In addition, the results of a Korean phase III trial of 12 weeks of sofosbuvir and ribavirin treatment demonstrated an SVR rate of $97 \% .{ }^{309}$ Extending treatment in patient with advanced fibrosis is short of solid evidences. Although the FISSION study reported liver cirrhosis to be a significant adverse factor for an SVR, the analysis was not stratified for genotype 2 patients. ${ }^{259}$ On the hands, another study suggested that treatment-experienced genotype 2 patients may benefit from extending treatment to 16 weeks, overcoming the negative effect of liver cirrhosis. ${ }^{310}$ Another Korean phase III trial reported an SVR rate of $100 \%$ in 13 cirrhotic genotype 2 patients. ${ }^{309}$ However, since liver cirrhosis inhibits achieving an SVR in many cases, extending treatment to 16 weeks in cirrhotic genotype $2 \mathrm{HCV}$ patients is recommended until more data are available. Reports of daclatasvir and sofosbuvir treatment in chronic HCV genotype 2 patients are scarce. Two recent studies involving treatment of HIV and HCV genotype 2 coinfected treatment-naïve patients for 12 or 24 weeks reported $100 \%$ SVR rates. ${ }^{234,311}$ Since the numbers of patients in these studies were 11 and 14, respectively, evidence supporting the use of daclatavir and sofosbuvir is insufficient. However, in treatmentnaïve HCV genotype 2 patients in whom ribavirin cannot be used, 12 weeks of treatment with daclatavir and sofosbuvir may be an alternative regimen.

PegIFN- $\alpha$ based therapy in treatment naïve genotype 2 patients consists of any one of two PegIFN- $\alpha$ combined with ribavirin for 24 weeks. ${ }^{254,261,262,312-314}$ PegIFN- $\alpha$ 2a $180 \mu$ g should be injected subcutaneously once a week, regardless of body weight, whereas PeglFN- $\alpha 2 \mathrm{~b}$ is to be injected $1.5 \mu \mathrm{g} / \mathrm{kg}$ subcutaneously once a week. Ribavirin is to be given at a flat dose of $800 \mathrm{mg}$ daily, regardless of the type of PegIFN- $\alpha$ used. ${ }^{261,262,312}$ There is a lack of evidence showing that a weight-based dose of ribavirin is more effective in achieving SVR for HCV genotype 2 patients. The SVR rate of HCV genotype 2 Korea patients treated with PegIFN- $\alpha$ and ribavirin exceeded $80 \% .{ }^{296,315}$ Regarding factors predicting relapse after treatment other than duration of therapy, existence of cirrhosis, baseline high viral load, body weight, gender, and old age have been suggested. ${ }^{266,316-318}$ However, studies contradicting these results also exist, and further research is required. ${ }^{319-321}$ Due to the high SVR rate after PegIFN- $\alpha$ and ribavirin combination treatment in genotype 2 patients, studies on factors predicting an SVR may require a relatively large sample size. ${ }^{322}$ A recent largescale study reported that advanced liver fibrosis had a significant effect on achieving an SVR. ${ }^{323}$ Studies of shortened treatment duration in PegIFN- $\alpha$ and ribavirin combination therapy are of heterogeneous design regarding treatment duration, ribavirin dose and use of RVR, and thus should not be compared directly. ${ }^{266,316,317,319,320,324-326}$ A study comparing 16 and 24 weeks of therapy in which each group contained $\sim 350$ patients, reported an SVR rate of $65 \%$ in the 16 -week treatment group compared to $82 \%$ in the 24 -week treatment group. ${ }^{320}$ However, shortening of the treatment duration was not performed according to the ontreatment response, RVR, instead being randomly assigned in this study. Another study compared 16- and 24-week treatment groups of $200 \mathrm{HCV}$ genotype 2 patients each, and reported an SVR rate of $81 \%$ in the 16 -week group and $92 \%$ in the 24 -week group, with no significant difference. ${ }^{316}$ However, the 16 -week treatment group had a relapse rate of $17 \%$, which was significantly higher than that of the 24-week treatment group, 5\%. ${ }^{316}$ In addition, it should be noted that this study used weight-based dose of ribavirin, which still resulted in a higher relapse rate when the treatment was shortened. In conclusion, patients with genotype 2 who achieve a RVR may have their duration of therapy shortened to 16 weeks only if there are no adverse predictors of response, such as advanced liver fibrosis, cirrhosis, and high baseline viral load, at the expense of a higher probability of post-treatment relapse. In patients with advanced liver fibrosis or cirrhosis, little evidence supports equal efficacy of shortened therapy. Studies 
The Korean Association for the Study of the Liver (KASL) KASL clinical practice guidelines: Management of Hepatitis C

of the efficacy of extended treatment up to 48 weeks in patients with negative predictors of a response have been performed. One study involving 1,311 HCV genotype 2 or 3 patients with negative predictors for SVR reported no benefit of extension of the treatment duration to 48 weeks in terms of achieving SVR. ${ }^{262}$

\section{Retreatment of treatment-experienced patients}

HCV genotype 2 patients in whom prior treatment with PegIFN- $\alpha$ and ribavirin failed can be retreated with sofosbuvir plus weight-based ribavirin. In a study in which treatment-experienced patients were given 12 weeks of sofosbuvir and ribavirin, the SVR rate was 93\% (68/73). ${ }^{327}$ This study reported no significant difference in SVR rate according to the existence of liver cirrhosis or pre-treatment serum HCV RNA level. Another study compared 12 and 16 weeks of treatment, and reported SVR rates of $86.1 \%(31 / 36)$ and $93.8 \%$ (30/32) (95\% confidence interval $(\mathrm{Cl}),-24 \%-9 \%)$, respectively. ${ }^{310}$ When cirrhotic patients were analyzed separately, the SVR rates after 12 and 16 weeks of treatment were $60.0 \%(6 / 10)$ and $77.8 \%(7 / 9)(95 \% \mathrm{Cl},-58.0 \%$ $26.8 \%)$, respectively, whereas those in non-cirrhotic patients were $96.2 \%(25 / 26)$ and $100 \%(23 / 23)(95 \% \mathrm{Cl},-20.2-11.3 \%){ }^{310}$ The BOSON study, in which sofosbuvir and ribavirin were given 16 or 24 weeks, reported an SVR rate of 94\% (15/16) and 100\% $(17 / 17) .{ }^{337}$ In contrast, a phase III from Korea and Taiwan reported an SVR rate of $100 \%(66 / 66)$ after 12 weeks of sofosbuvir and ribavirin treatment, regardless of accompanying liver cirrhosis. ${ }^{309}$ A Japanese study of 12-week treatment of sofosbuvir plus ribavirin showed an SVR rate of 95\% (60/63) in treatment-experienced HCV genotype 2 patients. ${ }^{328}$ This was comparable with the result in treatment-naïve Japanese patients, which was 98\% (88/90). ${ }^{328}$ The presence of liver cirrhosis did not significantly affect the SVR rate. Current studies do not provide strong support for extending treatment duration in treatment-experienced, cirrhotic genotype 2 patients undergoing sofosbuvir plus ribavirin treatment. However, both experiencing treatment failure in prior therapy and having liver cirrhosis are important adverse factors for achieving an SVR, and extending treatment for $16-24$ weeks is recommended until more data contradicting the benefit of this extension accumulate.

Data on daclatavir plus sofosbuvir combination therapy in treatment-experienced genotype 2 patients are limited. Achievement of an SVR in two HIV and HCV genotype 2 co-infected patients whose prior treatment failed has been reported. ${ }^{311}$ However, in cases in which ribavirin cannot be administered, 12 weeks of daclatavir plus sofosbuvir may be considered.

Another treatment option for HCV genotype 2 patients with a prior treatment failure is PegIFN, sofosbuvir and weight-based ribavirin for 12 weeks. A study on this combination reported an SVR rate of $96 \%$ (22/23), which was not affected by the presence of liver cirrhosis. ${ }^{310}$

Retreatment with PegIFN plus ribavirin can be considered in patients treated previously with conventional interferon with or without ribavirin, or PegIFN- $\alpha$ without ribavirin, and who failed to achieve SVR. ${ }^{329-335}$ The SVR rate after retreatment with PegIFN and ribavirin in relapsers is reported to be $\sim 50-70 \%$. ${ }^{329,330,332,334,335}$ The treatment is recommended for 24 weeks and the benefit of extending treatment to 48 weeks in these patients is unclear. For those who fail to achieve a SVR after PegIFN and ribavirin treatment, retreatment using the same regimen is not recommended as the SVR rate is reported to be as low as $10 \%{ }^{336}$ Patients treated with either a conventional interferon-based regimen or PegIFN without ribavirin and failed to achieve an SVR can be retreated with the same DAA-containing regimen as can those in whom prior treatment with PegIFN plus ribavirin has failed.

\section{[Recommendations] (Table 8)}

\section{Initial treatment of HCV genotype 2 infection}

1. Patients infected with HCV genotype 2 without cirrhosis should be treated with daily weight-based ribavirin $(1,200$

Table 8. Treatment of HCV genotype 2 infection in chronic hepatitis or compensated cirrhosis

\begin{tabular}{|c|c|c|c|c|}
\hline & \multicolumn{2}{|c|}{ Treatment naïve } & \multicolumn{2}{|c|}{ PR experienced } \\
\hline & Chronic hepatitis & $\begin{array}{l}\text { Compensated } \\
\text { cirrhosis }\end{array}$ & Chronic hepatitis & $\begin{array}{l}\text { Compensated } \\
\text { cirrhosis }\end{array}$ \\
\hline Sofosbuvir+R & 12 wk & $16 w k$ & $12 w k$ & $16-24 w k$ \\
\hline Daclatasvir+Sofosbuvir & \multicolumn{2}{|c|}{$12 w k$} & \multicolumn{2}{|c|}{$12 w k$} \\
\hline Sofosbuvir+PR & & & \multicolumn{2}{|c|}{$12 w k$} \\
\hline PR & $16-24 w k$ & $24 w k$ & & \\
\hline
\end{tabular}

PR, pegylated interferon- $\alpha$ +ribavirin $800 \mathrm{mg}$ therapy; $\mathrm{R}$, weight-based ribavirin. 
$\mathrm{mg}$ in patients $\geq 75 \mathrm{~kg}, 1,000 \mathrm{mg}$ in patients $<75 \mathrm{~kg}$ ) and sofosbuvir (400 mg) for 12 weeks (A1). Extension of treatment to 16 weeks is recommended for patients with cirrhosis (C1).

2. Patients infected with HCV genotype 2 with or without cirrhosis can be treated with daily daclatavir $(60 \mathrm{mg})$ and sofosbuvir ( $400 \mathrm{mg}$ ) for 12 weeks (C1).

3. Treatment with PegIFN- $\alpha$ in combination with ribavirin can be given for 24 weeks. (A2) PegIFN- $\alpha$ 2a should be injected $180 \mu \mathrm{g}$ subcutaneous once a week, regardless of patient body weight and PegIFN- $\alpha 2 \mathrm{~b}$ is to be injected $1.5 \mu \mathrm{g} / \mathrm{kg} /$ week (A1). Daily administration of $800 \mathrm{mg}$ of ribavirin can be performed, regardless of body weight (A2). In patients with an RVR who lack negative predictors for SVR, shortening of treatment duration to 16 weeks can be considered (B2). However, shortening of treatment duration should be done with caution, since this can result in a higher relapse rate (A2).

Retreatment of treatment-experienced patients with HCV genotype 2

1. Treatment-experienced patients with HCV genotype 2 without cirrhosis should be treated with daily sofosbuvir (400 mg) and weight-based ribavirin (1,200 mg in patients $\geq$ $75 \mathrm{~kg}, 1,000 \mathrm{mg}$ in patients $<75 \mathrm{~kg}$ ) for 12 weeks (A1). Duration of therapy should be extended to $16-24$ weeks in patients with cirrhosis (A1).

2. Treatment-experienced patients with HCV genotype 2 with or without cirrhosis can be treated with daily daclatavir $(60$ $\mathrm{mg}$ ) and sofosbuvir (400 mg) for 12 weeks (C1).

3. Treatment-experienced patients with HCV genotype 2 with or without cirrhosis can be treated with daily sofosbuvir (400 mg) and weight-based ribavirin (1,200 mg in patients $\geq$ $75 \mathrm{~kg}, 1,000 \mathrm{mg}$ in patients $<75 \mathrm{~kg}$ ) plus weekly PeglFN- $\alpha$ for 12 weeks (B1).

\section{TREATMENT OF CHRONIC HCV GENOTYPE 3 INFECTION}

\section{Initial treatment}

In a phase III study, 12 weeks of a daily sofosbuvir and weightbased ribavirin regimen for treatment-naïve patients with HCV genotype 3 infection was compared with a PegIFN- $\alpha$ and ribavirin combination regimen. The SVR rates were 56\% (102/183) and
$63 \%(110 / 176)$, respectively, which was not significantly different. ${ }^{259}$ In contrast, in another phase III study, 105 treatment-naïve patients with HCV genotype 3 infection were administered sofosbuvir and ribavirin for 24 weeks, and showed an SVR of $94 \%$ $(99 / 105){ }_{1}^{327}$ which was superior to that of 12 or 16 weeks of treatment $(62-63 \%) .{ }^{259,337}$ Since there were no differences in SVR rates according to the existence of liver cirrhosis in treatment-naïve patients with HCV genotype 3 infection, extension of the treatment duration might not be necessary. ${ }^{327}$

A daily daclatasvir and sofosbuvir combination regimen for 12 weeks showed an SVR of 89\% (16/19) in a phase II study of 19 treatment-naïve patients with HCV genotype 3 infection. ${ }^{234}$ In a phase III study of 75 treatment-naïve genotype 3 patients, this regimen showed a good SVR of $97 \%(73 / 75) .{ }^{338}$ However, extension of the treatment period was to be considered in treatmentnaive patients with cirrhosis due to a significantly lower SVR of $58 \%(11 / 19){ }^{339}$ A recent European cohort study reported that a daclatavir and sofosbuvir regimen for 24 weeks resulted in a significantly higher SVR of $88 \%(52 / 59)$ than did 12 weeks of treatment, which had an SVR rate of $76 \%(22 / 29){ }^{339}$ Therefore, it was suggested that extension of treatment is beneficial in treatmentnaïve cirrhotic patients. In addition, this study allowed addition of ribavirin to daclatavir and sofosbuvir according to the practitioner's demand, and addition of ribavirin to patients with negative predictors of SVR might be beneficial. ${ }^{339}$

Ledipasvir/sofosbuvir plus ribavirin for 12 weeks showed an excellent SVR of $100 \%$, compared to ledipasvir/sofosbuvir without ribavirin for 12 weeks, which had an SVR rate of $64 \% .{ }^{340}$ However, since an in vitro pharmacodynamic study of ledipasvir reported lower suppression of HCV genotype 3, further research on this regimen is warranted. ${ }^{340}$

PegIFN, sofosbuvir and weight-based ribavirin for 12 weeks showed an SVR of $90 \%$ in a phase II study of 10 non-cirrhotic and treatment-naïve HCV genotype 3 patients. ${ }^{341,342}$ In a phase III study of 123 treatment-naïve patients, this regimen showed a high SVR of $95 \%(117 / 123) .{ }^{340}$ Moreover, since there was no significant difference in SVR rate between patients with and without liver cirrhosis (SVR of $91 \%$ vs. $96 \%$, respectively), this regimen could be a treatment option for cirrhotic patients. ${ }^{342}$

The combination one of two PegIFN- $\alpha$ and ribavirin for 24 weeks in treatment-naïve HCV genotype 3 patients could be an alternative treatment regimen. ${ }^{254,261}$ PegIFN- $\alpha$ 2a $180 \mu \mathrm{g}$ should be injected subcutaneously once a week, regardless of body weight, whereas PegIFN- $\alpha$ 2b should be injected $1.5 \mu \mathrm{g} / \mathrm{kg}$ subcutaneously once a week. Ribavirin is to be given at a flat dose of $800 \mathrm{mg}$ daily, regardless of the type of PegIFN- $\alpha$ used. Evidence 
The Korean Association for the Study of the Liver (KASL) KASL clinical practice guidelines: Management of Hepatitis C

that a weight-based dose of ribavirin is more effective in achieving SVR in HCV genotype 3 patients is lacking. ${ }^{262,314}$ Although the SVR rate of HCV genotype 3 Korean patients has rarely been reported, HCV genotype 3 patients of other ethnicities showed a $10-20 \%$ lower SVR rate than genotype 2 patients. ${ }^{319,320,325}$

\section{Retreatment of treatment-experienced patients}

In a phase III study, sofosbuvir plus ribavirin treatment for 12 weeks was compared with that for 16 weeks in treatment-experienced HCV genotype 3 patients. The SVR rate was 30\% (19/64) after 12 weeks of treatment and 62\% (39/62) after 16 weeks of treatment. ${ }^{310}$ Furthermore, only 5 of 26 cirrhotic patients achieved an SVR (19\%). ${ }^{310}$ Although extending treatment to 24 weeks increased the SVR rate to $85 \%(213 / 250)$, cirrhotic and treatmentexperienced patients exhibited a low SVR rate of $62 \%$ (29/47) in a sub-group analysis. ${ }^{327}$ Thus a better treatment option is needed, especially for treatment-experienced, cirrhotic patients.

In a phase II study, treatment-experienced patients with genotype 3 infection received PegIFN, sofosbuvir and weight-based ribavirin for 12 weeks, and achieved an SVR rate of $83 \%$ (20/24). ${ }^{343}$ Of note, more than $50 \%$ of the patients in this study had liver cirrhosis and an 83\% (10/12) SVR was obtained, which was comparable to that of non-cirrhotic patients. ${ }^{343}$ In a phase III study of 544 treatment-experienced patients, PegIFN, sofosbuvir and weight-based ribavirin for 12 weeks resulted in an SVR rate of 93\% (166/181). ${ }^{337}$ This was higher than the SVR rate of sofosbuvir and ribavirin for 24 weeks which was 85\% (153/182). Of note, cirrhotic and treatment-experienced patients showed an SVR rate of $91 \%(21 / 23)$, which was comparable to that $96 \%$ (68/71) of noncirrhotic and treatment-experienced patients. ${ }^{337}$

Daily daclatasvir and sofosbuvir combination therapy for 12 weeks showed a SVR rate of $94 \%$ (32/34) in a phase III study of 34 treatment-experienced patients with HCV genotype 3 infection. ${ }^{338}$ However, cirrhotic and treatment-experienced patients achieved a lower SVR rate, 69\% (9/13). ${ }^{338}$ Consequently, when cirrhotic and treatment-experienced patients are treated, additional management such as add-on ribavirin or extension of the treatment period should be considered. A recent European cohort study reported that a daclatavir and sofosbuvir combination regimen for 24 weeks demonstrated a significantly higher SVR of $88 \%(52 / 59)$ compared to $76 \%(22 / 29)$ after 12 weeks of treatment. It was suggested that extension of treatment duration would benefit cirrhotic patients. ${ }^{339}$

Ledipasvir/sofosbuvir plus ribavirin combination therapy, which was suggested as an alternative treatment for treatment-experienced HCV genotype 3 patients, demonstrated an SVR rate of $82 \%$ (41/50). However, this regimen resulted in a lower SVR rate of $73 \%$, and so further studies are needed. ${ }^{340}$

Retreatment with PegIFN plus ribavirin can be considered in patients previously treated with conventional interferon with or without ribavirin, or PegIFN- $\alpha$ without ribavirin, who failed to achieve SVR. ${ }^{329-332,344}$ Patients treated with either a conventional interferonbased regimen or PegIFN without ribavirin and who failed to achieve an SVR can be retreated with the same DAA containing regimen as those in whom prior treatment with PegIFN plus ribavirin failed.

\section{[Recommendations] (Table 9)}

\section{Initial treatment of HCV genotype 3 infection}

1. Patients infected with HCV genotype 3 without cirrhosis should be treated with daily sofosbuvir (400 mg) and weight-based ribavirin $(1,200 \mathrm{mg}$ in patients $\geq 75 \mathrm{~kg}, 1,000$ $\mathrm{mg}$ in patients $<75 \mathrm{~kg}$ ) for 24 weeks (A1). The same regimen is recommended for patients with cirrhosis (B1).

2. Patients infected with HCV genotype 3 without cirrhosis should be treated with daily daclatasvir $(60 \mathrm{mg})$ and sofosbuvir ( $400 \mathrm{mg}$ ) for 12 weeks (A1). Extending treatment to 24 weeks with or without adding ribavirin is recommended for patients with cirrhosis (B2).

3. Patients infected with HCV genotype 3 without cirrhosis

Table 9. Treatment of HCV genotype 3 infection in chronic hepatitis or compensated cirrhosis

\begin{tabular}{|c|c|c|c|c|}
\hline & \multicolumn{2}{|c|}{ Treatment naïve } & \multicolumn{2}{|c|}{ PR experienced } \\
\hline & Chronic hepatitis & Compensated cirrhosis & Chronic hepatitis & Compensated cirrhosis \\
\hline Sofosbuvir+R & \multicolumn{2}{|c|}{$24 w k$} & \multicolumn{2}{|l|}{$24 w k$} \\
\hline Daclatasvir+Sofosbuvir & $12 w k$ & $24 w k \pm R$ & $12 w k$ & $24 w k \pm R$ \\
\hline Sofosbuvir+PR & \multicolumn{2}{|c|}{$12 w k$} & \multicolumn{2}{|c|}{$12 w k$} \\
\hline PR & $16-24 w k$ & $24 w k$ & & \\
\hline
\end{tabular}

PR, pegylated interferon- $\alpha$ +ribavirin $800 \mathrm{mg}$ therapy; $\mathrm{R}$, weight-based ribavirin. 
should be treated with daily sofosbuvir $(400 \mathrm{mg})$ and weight-based ribavirin $(1,200 \mathrm{mg}$ in patients $\geq 75 \mathrm{~kg}, 1,000$ $\mathrm{mg}$ in patients $<75 \mathrm{~kg}$ ) plus weekly PegIFN- $\alpha$ for 12 weeks (A1). The same regimen is recommended for patients with cirrhosis (B1).

4. Treatment with PegIFN- $\alpha$ in combination with ribavirin can be given for 24 weeks (A2). PegIFN- $\alpha$ 2a should be injected $180 \mu \mathrm{g}$ subcutaneous once a week, regardless of patient body weight and PegIFN- $\alpha 2 \mathrm{~b}$ is to be injected $1.5 \mu \mathrm{g} / \mathrm{kg} /$ week (A1). Daily administration of $800 \mathrm{mg}$ of ribavirin can be performed, regardless of body weight (A2).

Retreatment of treatment-experienced patients with HCV genotype 3 infection

1. Treatment-experienced patients with HCV genotype 3 with or without liver cirrhosis should be treated with daily sofosbuvir (400 mg) and weight-based ribavirin (1,200 mg in patients $\geq 75 \mathrm{~kg}, 1,000 \mathrm{mg}$ in patients $<75 \mathrm{~kg}$ ) plus weekly PegIFN- $\alpha$ for 12 weeks (A1).

2. Treatment-experienced patients with HCV genotype 3 without cirrhosis should be treated with daily daclatasvir (60 mg) and sofosbuvir (400 mg) for 12 weeks. (A1) Extending treatment to 24 weeks with or without adding ribavirin is recommended for patients with cirrhosis (B2).

3. Treatment-experienced patients with HCV genotype 3 without cirrhosis can be treated with daily sofosbuvir (400 $\mathrm{mg}$ ) and weight-based ribavirin (1,200 $\mathrm{mg}$ in patients $\geq 75$ $\mathrm{kg}, 1,000 \mathrm{mg}$ in patients $<75 \mathrm{~kg}$ ) for 24 weeks (B2). Due to the suboptimal sustained virological response for treatment-experienced patients with cirrhosis, an alternative regimen should be considered in these patients (B1).

\section{TREATMENT OF CHRONIC HCV GENOTYPE 4 INFECTION}

There are no reports of the therapeutic outcome of patients infected with HCV genotype 4 in South Korea. The SVR rate was reported to be $72 \%$ in patients infected with HCV genotype 4 treated with the combination of PegIFN- $\alpha$ and ribavirin for 48 weeks. $^{345}$

Several IFN-free regimens were evaluated for treatment of patients infected with HCV genotype 4.

Three trials, in which patients infected with HCV genotype 4 treated for 24 weeks with sofosbuvir and weight-based ribavirin exhibited SVR rates of 93\% (27/29), 90\% (46/51) and 84\% (26/31), respectively. ${ }^{346-348}$ The SVR rate was decreased to $78 \%$ in cirrhotic patients treated with the same regimen. ${ }^{347}$ Another interferon-free regimen comprising ledipasvir/sofosbuvir for 12 weeks showed a 95\% SVR in 21 patients infected with HCV genotype 4, including 7 cirrhotic patients. ${ }^{349}$ SVR rates were 100\% (42/42) and $100 \%$ (49/49) in treatment-naïve and treatment-experienced patients infected with HCV genotype 4 treated for 12 weeks with ombitasvir/paritaprevir/ritonavir and weight-based ribavirin, respectively. ${ }^{350}$ The triple combination of daclatasvir, asunaprevir and beclabuvir resulted in a 100\% SVR in 21 treatment-naïve and non-cirrhotic patients infected with HCV genotype $4 .{ }^{350}$

Although the evidence is weak, it is likely that the results of several trials involving patients infected with genotype 1 can be extrapolated. Interferon-free regimens for the treatment of cirrhotic patients infected with HCV genotype 4 could be prolonged to 24 weeks or weight-based ribavirin added to improve the SVR rates.

The combination of PegIFN- $\alpha$, weight-based ribavirin and sofosbuvir for 12 weeks as an interferon-containing regimen showed a 96\% SVR rate in 28 treatment-naïve patients infected with HCV genotype $4 .{ }^{259}$ Another interferon-containing regimen comprising PegIFN- $\alpha 2$ a, weight-based ribavirin and simeprevir for 24 or 48 weeks showed an overall 65\% (70/107) SVR rate in patients infected with HCV genotype 4. Indeed, an SVR was achieved with this regimen in 83\% (29/35) of treatment-naïve patients, $86 \%(19 / 22)$ of prior relapsers, $60 \%(6 / 10)$ of prior partial responders, and $40 \%$ (16/40) of prior null responders. ${ }^{351}$ If DAAs are not available, the previous standard therapy such as the combination of PegIFN- $\alpha$ and ribavirin for 48 weeks remains acceptable.

\section{[Recommendations] (Table 10)}

\section{Initial treatment of HCV genotype 4 infection}

1. Patients infected with HCV genotype 4 without cirrhosis can be treated with daily ledipasvir $(90 \mathrm{mg}) / \mathrm{sofosbuvir} \mathrm{(400}$ $\mathrm{mg}$ ) for 12 weeks (B1). Duration of therapy can be extended to 24 weeks or daily weight-based ribavirin $(1,200 \mathrm{mg}$ in patients $\geq 75 \mathrm{~kg}, 1,000 \mathrm{mg}$ in patients $<75 \mathrm{~kg}$ ) can be added without extending treatment duration in patients with cirrhosis (C1).

2. Patients infected with HCV genotype 4 without cirrhosis can be treated with daily ombitasvir $(25 \mathrm{mg}) /$ paritaprevir (150 mg)/ritonavir (100 $\mathrm{mg}$ ) and weight-based ribavirin 
The Korean Association for the Study of the Liver (KASL) KASL clinical practice guidelines: Management of Hepatitis C

$(1,200 \mathrm{mg}$ in patients $\geq 75 \mathrm{~kg}, 1,000 \mathrm{mg}$ in patients $<75 \mathrm{~kg})$ for 12 weeks (B1). Treatment can be extended to 24 weeks in patients with cirrhosis (C1).

3. Patients infected with HCV genotype 4 without cirrhosis can be treated with daily sofosbuvir $(400 \mathrm{mg})$ and weightbased ribavirin $(1,200 \mathrm{mg}$ in patients $\geq 75 \mathrm{~kg}, 1,000 \mathrm{mg}$ in patients $<75 \mathrm{~kg}$ ) for 24 weeks (B1).

4. Patients infected with HCV genotype 4 with or without cirrhosis can be treated with daily sofosbuvir $(400 \mathrm{mg})$ and weight-based ribavirin $(1,200 \mathrm{mg}$ in patients $\geq 75 \mathrm{~kg}, 1,000$ $\mathrm{mg}$ in patients $<75 \mathrm{~kg}$ ) plus weekly PegIFN- $\alpha$ for 12 weeks (B1).

5. Patients infected with HCV genotype 4 with or without cirrhosis can be treated with PegIFN- $\alpha$ and weight-based ribavirin for 48 weeks (A2). PegIFN- $\alpha$ 2a should be injected 180 $\mu \mathrm{g}$ subcutaneous once a week, regardless of patient body weight with ribavirin using doses of $1,000 \mathrm{mg} / \mathrm{d}$ for those $\leq$ $75 \mathrm{~kg}$ in weight and 1,200 mg/day for those $>75 \mathrm{~kg}$. PegIFN- $\alpha 2 \mathrm{~b}$ is to be injected $1.5 \mu \mathrm{g} / \mathrm{kg} /$ week with ribavirin using doses of $800 \mathrm{mg}$ for those $<65 \mathrm{~kg}$ in weight, 1,000 $\mathrm{mg}$ for $65-85 \mathrm{~kg}, 1,200 \mathrm{mg}$ for $85-105 \mathrm{~kg}$, and 1,400 mg for $>$ $105 \mathrm{~kg}(\mathrm{~A} 1)$.

\section{TREATMENT OF CHRONIC HCV GENOTYPE 5 OR 6 INFECTION}

There is no report of HCV genotype 5 in South Korea, which is limited mostly to South Africa. HCV genotype 6 is limited mostly to Southeast Asia, Southern China, Hong Kong, and Macau. It comprises about $1 \%$ of total chronic HCV patients in South Korea. ${ }^{352}$ SVR rate of chronic HCV genotype 5 or 6 treated with a combination of PegIFN- $\alpha$ and ribavirin is $70-86 \%$, which is comparable with that of HCV genotype 3 and higher than that of HCV genotype $1 .{ }^{353-355}$ Studies for HCV genotype 5 or 6 are limited be- cause of the small number of patients infected with HCV genotype 5 or 6 .

The combination of ledipasvir/sofosbuvir for 12 weeks as an IFN-free DAA regimen showed a 96\% SVR in 25 treatment-naïve and -experienced patients infected with HCV genotype $6 .{ }^{340}$ This result can be extrapolated for treatment of HCV genotype 5, although there are no data regarding use of this combination in patients infected with HCV genotype 5 , because ledipasvir is active against both genotypes 5 and 6 in vitro.

Treatment with PegIFN- $\alpha$, weight-based ribavirin and sofosbuvir for 12 weeks achieved a 100\% SVR in one patient infected with HCV genotype 5 and six patients infected with HCV genotype $6 .{ }^{259}$

Due to the weak evidence, the results of several trials involving patients infected with genotype 1 can be extrapolated. IFN-free regimens for the treatment of cirrhotic patients infected with HCV genotype 5 or 6 could be prolonged to 24 weeks or weight-based ribavirin added to improve the SVR rate.

If DAAs are not available, the previous standard therapy such as the combination of PegIFN- $\alpha$ and ribavirin for 24 weeks remains acceptable.

\section{[Recommendations] (Table 10)}

Initial treatment of HCV genotype 5 or 6

1. Patients infected with HCV genotype 5 or 6 without cirrhosis can be treated with daily ledipasvir $(90 \mathrm{mg}) /$ sofosbuvir (400 mg) for 12 weeks (B1). Duration of therapy can be extended to 24 weeks or daily weight-based ribavirin $(1,200$ $\mathrm{mg}$ in patients $\geq 75 \mathrm{~kg}, 1,000 \mathrm{mg}$ in patients $<75 \mathrm{~kg}$ ) can be added without extending treatment duration in patients with cirrhosis (C1).

2. Patients infected with HCV genotype 5 or 6 with or without cirrhosis can be treated with daily sofosbuvir $(400 \mathrm{mg})$ and weight-based ribavirin $(1,200 \mathrm{mg}$ in patients $\geq 75 \mathrm{~kg}, 1,000$ $\mathrm{mg}$ in patients $<75 \mathrm{~kg}$ ) plus weekly PegIFN- $\alpha$ for 12 weeks

Table 10. Treatment of HCV genotype 4, 5, 6 infection in chronic hepatitis or compensated cirrhosis

\begin{tabular}{|c|c|c|c|c|}
\hline & \multicolumn{2}{|c|}{ Genotype 4} & \multicolumn{2}{|c|}{ Genotype 5, 6} \\
\hline & Chronic hepatitis & Compensated cirrhosis & Chronic hepatitis & Compensated cirrhosis \\
\hline Ledipasvir/sofosbuvir & $12 w k$ & $12 w k+R / 24 w k$ & $12 w k$ & $12 w k+R / 24 w k$ \\
\hline $\mathrm{OPr}+\mathrm{R}$ & $12 w k$ & $24 w k$ & & \\
\hline Sofosbuvir+R & $24 w k$ & & & \\
\hline Sofosbuvir+PR & \multicolumn{2}{|c|}{$12 w k$} & \multicolumn{2}{|r|}{$12 w k$} \\
\hline$P R$ & \multicolumn{2}{|c|}{$48 w k$} & \multicolumn{2}{|r|}{$24 w k$} \\
\hline
\end{tabular}

$\mathrm{R}$, weight-based ribavirin; OPr, ombitasvir/paritaprevir/ritonavir; PR, pegylated interferon- $\alpha$ +ribavirin therapy. 
(B1).

3. Patients infected with HCV genotype 5 or 6 with or without cirrhosis can be treated with PegIFN- $\alpha$ and weight-based ribavirin for 24 weeks (A2). PegIFN- $\alpha$ 2a should be injected $180 \mu \mathrm{g}$ subcutaneous once a week, regardless of patient body weight with ribavirin using doses of $1,000 \mathrm{mg} / \mathrm{d}$ for those $\leq 75 \mathrm{~kg}$ in weight and 1,200 mg/day for those $>75 \mathrm{~kg}$. PegIFN- $\alpha 2 b$ is to be injected $1.5 \mu \mathrm{g} / \mathrm{kg} /$ week with ribavirin using doses of $800 \mathrm{mg}$ for those $<65 \mathrm{~kg}$ in weight, 1,000 $\mathrm{mg}$ for $65-85 \mathrm{~kg}, 1,200 \mathrm{mg}$ for $85-105 \mathrm{~kg}$, and 1,400 $\mathrm{mg}$ for $>$ $105 \mathrm{~kg}(\mathrm{~A} 1)$.

\section{TREATMENT OF PATIENTS WITH DECOMPEN- SATED CIRRHOSIS}

In the SOLAR-2 study, which was a multicenter randomized controlled trial of 108 patients with HCV genotypes 1 and 4 with decompensated cirrhosis (Child-Pugh Turcotte [CTP] class B or C, CTP scores $\leq 12$ ), participants were randomly assigned to receive daily fixed-dose combination ledipasvir (90 mg) and sofosbuvir (400 mg) and ribavirin (initial dose of $600 \mathrm{mg}$, increased as tolerated) for 12 or 24 weeks. ${ }^{356}$ SVR was achieved in $87 \%$ and $89 \%$ of patients given the 12- and 24-week treatment courses, respectively. Baseline CTP and Model for End-Stage Liver Disease (MELD) scores improved in more than $50 \%$ of the patients, but some patients experienced worsening of hepatic function. During the treatment, five (5\%) patients died of variceal bleeding. Grade 3 or 4 adverse events occurred in $15 \%$ and $34 \%$ in the 12 - and 24 -week arms, respectively.

In the phase III ALLY-1 study, daclatasvir (60 mg) was administered daily in combination with sofosbuvir $(400 \mathrm{mg}$ ) and a low initial dose of ribavirin $(600 \mathrm{mg}$ ) for 12 weeks to 60 patients with decompensated cirrhosis (mostly CTP class B and C, HCV genotype 1:3:2/4/6=45:6:9). ${ }^{357}$ The overall SVR rate was $83 \%$. SVR rates were $76 \%$ and $100 \%$ among patients with HCV genotypes $1 \mathrm{a}$ and $1 \mathrm{~b}$, respectively. In patients with HCV genotype 1 , SVR rates were $92 \%$ and $50 \%$ among patients with CTP classes B and C, respectively. Among subjects with HCV genotype 3 and 2/4/6, SVR12 rates were $83 \%$ and $89 \%$, respectively.

The efficacy and safety of simeprevir plus sofosbuvir with or without ribavirin for 12 weeks were assessed in patients with CTP class $B / C(n=55)$ versus CTP class A ( $n=101)$ cirrhosis and compared to matched untreated controls. ${ }^{358}$ SVR was achieved by $73 \%$ of CTP class B/C versus $91 \%$ of CTP class A $(P<0.01)$ patients. CTP class B/C vs. CTP class A patients had more early treatment discontinuations (11\% vs. 1\%), adverse events requiring hospitalization (22\% vs. $2 \%$ ), infections requiring antibiotics ( $20 \%$ vs. $1 \%)$, and hepatic decompensating events ( $20 \%$ vs. $3 \%$; all $P<0.01$ ). Adverse events requiring hospitalization (9\% vs. $13 \%$; $P=0.55)$, infections ( $8 \%$ vs. $6 \% ; P=0.47)$, and events of decompensation ( $9 \%$ vs. $10 \% ; P=0.78$ ) occurred at similar frequency in simeprevir plus sofosbuvir-treated patients and matched untreated controls. Use of ribavirin with simeprevir plus sofosbuvir was not associated with an SVR.

Among DAAs, paritaprevir, dasabuvir and asunaprevir are contraindicated in patients with decompensated cirrhosis due to the higher drug concentrations observed.

Efficacy and safety data in patients with more advanced liver disease (Child-Pugh $\geq 13$ ) are limited and require verification. Also, the long-term clinical benefit of antiviral therapy in patients with decompensated cirrhosis without consideration for liver transplantation is unclear; thus it should be individualized.

Therapeutic outcome with PegIFN- $\alpha$ plus ribavirin in decompensated cirrhotic patients is very poor, with frequent treatment-related complications. A small study ( $n=10,8$ genotype $1 \mathrm{HCV}$ patients, 2 genotype non-1 HCV patients) reported a SVR rate of $20.0 \%$ in treating decompensated cirrhotic patients using combination therapy of PegIFN- $\alpha$ plus ribavirin. ${ }^{359}$ Therefore, antiviral treatment of CTP class B cirrhotic patients can be attempted by experienced specialists with careful monitoring. A relatively good therapeutic outcome is expected in cases of low HCV RNA concentrations, or HCV genotype 2 or 3 . Although drug administration can be started with standard doses, more than half of the

Table 11. Treatment of decompensated cirrhosis

\begin{tabular}{lccc}
\hline & Genotype 1, 4, 5, 6 & Genotype 2 & Genotype 3 \\
\hline Ledipasvir/sofosbuvir & $12 w k+R^{*} / 24 w k$ & & $12 w k+R^{*} / 24 w k$ \\
\hline Daclatasvir+Sofosbuvir & $12 w k+R^{*} / 24 w k$ & $12 w k+R^{*} / 24 w k$ & $24-48 w k$ \\
Sofosbuvir+Simeprevir & $12 w k$ & & $16-24 w k$ \\
Sofosbuvir+R & & & \\
\hline
\end{tabular}

$R^{*}$, ribavirin started from $600 \mathrm{mg} / \mathrm{d}$; $R$, weight-based ribavirin. 
The Korean Association for the Study of the Liver (KASL) KASL clinical practice guidelines: Management of Hepatitis C

patients experienced drug discontinuation or dose reduction. Thus, a careful approach starting with a low accelerated dose regimen (starting with $90 \mu \mathrm{g} /$ week of PegIFN- $\alpha$ 2a or $0.5 \mu \mathrm{g} / \mathrm{kg}$ / week of PegIFN- $\alpha 2 b$ and $600 \mathrm{mg} /$ day of ribavirin, and a gradual increase in dose every 2 weeks up to the maximum tolerable dose) was assessed, and showed a similar efficacy to a standard dose regimen. ${ }^{244}$ The combination treatment with PegIFN- $\alpha$ plus ribavirin is contraindicated in patients with CTP class C due to the likelihood of severe complications, including death. ${ }^{244}$

\section{[Recommendations] (Table 11)}

Interferon-free regimen

1. Treatment of patients infected with HCV genotypes $1,4,5$ or 6 who have decompensated cirrhosis

1) Daily fixed-dose combination ledipasvir $(90 \mathrm{mg}) / \mathrm{sofos}$ buvir (400 mg) and a low initial dose of ribavirin (600 $\mathrm{mg}$, increased as tolerated) for 12 weeks is recommended for patients with HCV genotype 1, 4, 5 or 6 infection who have decompensated cirrhosis (B1). Daily fixeddose combination ledipasvir $(90 \mathrm{mg}) /$ sofosbuvir (400 $\mathrm{mg}$ ) for 24 weeks can be used for patients who are ribavirin intolerant or ineligible (C1).

2) Daily daclatasvir (60 mg), sofosbuvir (400 mg), and a low initial dose of ribavirin (600 $\mathrm{mg}$, increased as tolerated) for 12 weeks is recommended for patients with HCV genotype 1, 4, 5 or 6 infection who have decompensated cirrhosis (B1). Daily daclatasvir (60 mg) and sofosbuvir (400 mg) for 24 weeks can be used for patients who are ribavirin intolerant or ineligible (C1).

3) Daily sofosbuvir (400 mg) and simeprevir (150 mg) for 12 weeks can be used for patients with HCV genotype 1, 4, 5 or 6 infection who have decompensated cirrhosis (C2).

2. Treatment of patients infected with HCV genotype 2 who have decompensated cirrhosis

1) Daily sofosbuvir (400 mg) and weight-based ribavirin $(1,200 \mathrm{mg}$ in patients $\geq 75 \mathrm{~kg}, 1,000 \mathrm{mg}$ in patients < $75 \mathrm{~kg}$ ) for 16-24 weeks can be used for patients with HCV genotype 2 infection with decompensated cirrhosis (C1).

2) Daily daclatasvir (60 mg), sofosbuvir (400 mg), and a low initial dose of ribavirin (600 mg, increased as tolerated) for 12 weeks can be used for patients with HCV genotype 2 infection with decompensated cirrhosis (C1). Daily daclatasvir (60 mg) and sofosbuvir (400 mg) for 24 weeks can be used for patients who are ribavirin intolerant or ineligible (C1).

3. Treatment of patients with HCV genotype 3 who have decompensated cirrhosis

1) Daily daclatasvir (60 mg), sofosbuvir (400 mg), and a low initial dose of ribavirin (600 $\mathrm{mg}$, increased as tolerated) for 12 weeks can be used for patients with HCV genotype 3 infection with decompensated cirrhosis (C1). Daily daclatasvir (60 mg) and sofosbuvir (400 mg) for 24 weeks can be used for patients who are ribavirin intolerant or ineligible (C1).

2) Daily sofosbuvir (400 mg) and weight-based ribavirin $(1,200 \mathrm{mg}$ in patients $\geq 75 \mathrm{~kg}, 1,000 \mathrm{mg}$ in patients $<75$ $\mathrm{kg}$ ) for 24-48 weeks can be used for patients with HCV genotype 3 infection with decompensated cirrhosis (C2).

Interferon-containing regimen

In CTP class B cirrhotic patients, combination treatment with PegIFN- $\alpha$ plus ribavirin can be attempted by experienced specialists with careful monitoring (C2). Combination treatment with PegIFN- $\alpha$ plus ribavirin is contraindicated in patients of CTP class C due to the likelihood of severe complications, including death (B1).

\section{LIVER TRANSPLANTATION AND OTHER OR- GAN TRANSPLANTS}

\section{Treatment before liver transplantation}

HCV reinfection occurs within several hours after transplantation in most patients with detectable HCV RNA at the time of the transplantation. ${ }^{360}$ Thus, patients with HCV infection at the time of liver transplantation have a higher graft failure rate (hazard ratio (HR), 1.30; 95\% Cl, 1.21-1.39) and mortality rate (HR, 1.23; $95 \% \mathrm{Cl}, 1.12-1.35)$ compared to patients without HCV infection. ${ }^{361}$ HCV-related liver diseases rapidly deteriorate following liver transplantation and around one-third of patients progress to cirrhosis within 5 years after transplantation. ${ }^{361,362}$ Therefore, successful elimination of HCV before or after transplantation is critical to improve the prognosis of the graft and patient.

Treatment of HCV infection in patients awaiting a liver transplantation has two goals: i) preventing liver graft infection after transplantation, and ii) improving liver function before transplantation in patients with decompensated cirrhosis. Improvement of 
liver function may lead to delisting of some of patients awaiting transplantation. ${ }^{363}$ However, the duration of antiviral therapy cannot be predicted in a patient on the waiting list.

Curry et al. reported a study in which 61 patients infected with HCV genotype 1, 2, 3 or 4 were treated with sofosbuvir and ribavirin up to 48 weeks prior to transplantation; 46 of them were transplanted. ${ }^{364}$ Among 43 patients with an HCV RNA level $<25$ $\mathrm{IU} / \mathrm{ml}$ at the time of transplantation, $30(70 \%)$ had an SVR posttransplantation. The duration of undetectable HCV RNA pretransplant was the best predictor of a response. HCV recurrence occurred in $64 \%(9 / 14)$ vs. $4 \%(1 / 26)$ in patients with undetectable HCV RNA for less than 30 continuous days vs. more than 30 days before transplantation, respectively.

\section{Treatment following liver transplantation}

All patients with post-transplant recurrence of HCV infection should be prioritized for antiviral therapy. In particular, antiviral treatment should be started as soon as possible when advanced fibrosis or portal hypertension is noted, since these conditions predict a rapid progression of liver diseases and graft failure.

The SOLAR-1 study was a large, multicenter, randomized controlled trial that included liver transplant recipients $(n=222)$ infected with HCV genotype 1 or 4 . Study participants were randomly assigned to receive fixed-dose combination ledipasvir (90 $\mathrm{mg}$ ) and sofosbuvir (400 mg) and weight-based ribavirin (1,000 $\mathrm{mg}[<75 \mathrm{~kg}]$ to $1,200 \mathrm{mg}[>75 \mathrm{~kg}])$ for either 12 weeks or 24 weeks. $^{359}$ In patients with Metavir fibrosis stages F0 to F3, SVR was achieved in $96 \%$ and $98 \%$ of patients in the 12 - and 24 week arms, respectively. In patients with compensated cirrhosis, SVR was achieved in $96 \%$ of patients in both the 12- and 24 week arms. Efficacy was lower in patients with CTP class B cirrhosis (SVR $85 \%$ vs. $88 \%$ in the 12 - and 24 -week arms) or CTP class C cirrhosis (60\% vs. $75 \%$ in the 12 - and 24 -week arms).

A total of 123 patients infected with HCV genotype 1 received simeprevir and sofosbuvir with or without ribavirin treatment for
12 weeks. ${ }^{365}$ An SVR was achieved in $90 \%$ of patients, and the addition of ribavirin did not impact the SVR rate. Because of significantly increased plasma concentrations of simeprevir, the concomitant use of simeprevir and cyclosporine is not recommended in liver transplant recipients. No simeprevir dose changes are required with tacrolimus and sirolimus.

In the phase III ALLY-1 study, daclatasvir (60 mg daily) was administered in combination with daily sofosbuvir (400 mg) and ribavirin (initial dose, $600 \mathrm{mg}$ ) for 12 weeks to patients with recurrent HCV infection post-transplant $(n=53, H C V$ genotype 1:3:2/4/6=41:11:1). ${ }^{360}$ The SVR rate was $94 \%$. SVR rates were $95 \%, 91 \%$ and $100 \%$ in patients infected with genotypes 1,3 and $2 / 4 / 6$, respectively.

In a multicenter study of 34 liver transplant recipients with mild recurrence (Metavir fibrosis stage F0-F2) of HCV genotype 1 infection, fixed-dose combination paritaprevir (150 mg), ritonavir (100 mg), and ombitasvir (25 mg) plus twice-daily dosed dasabuvir (250 mg) and weight-based ribavirin was given for 24 weeks, and achieved an SVR rate of $97 \% .{ }^{366}$ Two patients (6\%) had serious adverse events, and there were no deaths during the study. Because of interactions with ritonavir and paritaprevir, adjustments of the cyclosporine and tacrolimus dose were needed.

When interferon-containing treatment is considered in patients with HCV reinfection following liver transplantation, it is recommended after histological confirmation of chronic hepatitis $C$ at least 6 months after transplantation. This is because shortly after transplantation, patients are heavily immunosuppressed and incompletely recovered from the surgery, resulting in a high probability of drug intolerability as well as allograft rejection during interferon use.

The SVR rate of interferon-containing antiviral treatment after transplantation is $30-40 \%$, and genotypes 2 and 3 show better therapeutic outcomes than genotype $1^{245,367,368}$ Post-transplant patients reportedly show similar therapeutic outcomes (33\% and $38 \%$ SVR rates, respectively) using PegIFN- $\alpha$ plus ribavirin combination therapy or PegIFN- $\alpha$ monotherapy, which may be due to frequent dose reduction or discontinuation of ribavirin due to

Table 12. Treatment after liver transplantation

\begin{tabular}{lcccc}
\hline & Genotype 1 & Genotype 4 & Genotype 2 & Genotype 3 \\
\hline Ledipasvir/sofosbuvir & $12 w k+R / 24 w k$ & $12 w k+R / 24 w k$ & & $12 w k+R^{*} / 24 w k$ \\
Daclatasvir+Sofosbuvir & $12 w k+R^{*} / 24 w k$ & $12 w k+R^{*} / 24 w k$ & $12 w k+R^{*} / 24 w k$ & \\
Sofosbuvir+Simeprevir & $12 w k \pm R$ & $12 w k \pm R$ & & $24 w k$ \\
OPr+D or OPr & $(\mathrm{OPr}+\mathrm{D}) 24 w k+R$ & $(\mathrm{OPr}) 12-24 w k+R$ & $12-24 w k$ & $24 \mathrm{w}$ \\
Sofosbuvir+R & & & & \\
\hline
\end{tabular}

$R$, weight-based ribavirin; $R^{*}$, ribavirin started from $600 \mathrm{mg} / \mathrm{d}$; $\mathrm{OPr}+\mathrm{D}$, ombitasvir/paritaprevir/ritonavir+dasabuvir. 
The Korean Association for the Study of the Liver (KASL) KASL clinical practice guidelines: Management of Hepatitis C

complications. ${ }^{369}$ Anemia is the most common cause of treatment discontinuation and recombinant erythropoietin is recommended in this case. ${ }^{367,368}$ Allograft rejection related to interferon alpha use can occur, and liver biopsy is required to differentiate the cause of liver function deterioration during antiviral treatment.

\section{Treatment following other organ transplants}

Renal transplant patients with HCV infection display rapidly progressing hepatic fibrosis and show high mortality related to hepatic failure; thus, antiviral treatment was recommended prior to renal transplantation in the past. ${ }^{370}$ However, with the introduction of DAAs, successful elimination of HCV after renal transplantation could be achieved. It remains to be determined whether patients with chronic hepatitis $C$ should optimally proceed to renal transplantation, with the expectation that their hepatitis $C$ can be cured post-transplant to improve the outcome.

Combination therapy with PegIFN- $\alpha$ plus ribavirin causes graft rejection in over $30 \%$ of patients, leading to graft failure and death. Thus, DAA therapy is preferred over interferon-containing therapy in renal transplant patients. ${ }^{371,372}$ No data on the situation of transplants of the heart, lung, pancreas, small intestine, or cornea are available. When antiviral therapy is needed, DAA therapy is preferred over interferon-containing therapy.

\section{[Recommendations] (Table 12)}

1. Antiviral therapy can prevent graft infection in patients awaiting liver transplantation, and should follow the recommendations according to liver function and HCV genotype (B1).

2. All patients who develop recurrent HCV infection after liver transplantation should be prioritized for antiviral therapy (A1). Antiviral treatment should be started as soon as possible when fibrosing cholestatic fibrosis, advanced fibrosis or portal hypertension is noted, since these conditions predict a rapid progression of liver diseases and graft failure (A1).

3. Treatment of patients who develop recurrent HCV infection after liver transplantation: HCV genotype 1 or 4

1) Daily fixed-dose combination ledipasvir ( $90 \mathrm{mg}) / \mathrm{sofosbu}$ vir (400 mg) and weight-based ribavirin (1,200 mg in patients $\geq 75 \mathrm{~kg}, 1,000 \mathrm{mg}$ in patients $<75 \mathrm{~kg}$ ) for 12 weeks is recommended for post-liver transplantation patients infected with HCV genotypes 1 or 4 (B1). A low initial dose of ribavirin $(600 \mathrm{mg}$, increased as tolerated) is rec- ommended for patients with decompensated cirrhosis (B1). Daily fixed-dose combination ledipasvir $(90 \mathrm{mg}) / \mathrm{so}$ fosbuvir ( $400 \mathrm{mg}$ ) for 24 weeks can be used for patients who are ribavirin intolerant or ineligible (C1).

2) Daily daclatasvir (60 mg), sofosbuvir (400 mg), and a low initial dose of ribavirin (600 $\mathrm{mg}$, increased as tolerated) for 12 weeks is recommended for post-liver transplantation patients infected with HCV genotypes 1 or 4 (B1). Daily daclatasvir $(60 \mathrm{mg}$ ) and sofosbuvir $(400 \mathrm{mg}$ ) for 24 weeks can be used for patients who are ribavirin intolerant or ineligible (C1).

3) Daily sofosbuvir ( $400 \mathrm{mg}$ ) and simeprevir (150 mg) for 12 weeks is recommended for post-liver transplantation patients infected with HCV genotypes 1 or 4 (B1). Weightbased ribavirin $(1,200 \mathrm{mg}$ in patients $\geq 75 \mathrm{~kg}, 1,000 \mathrm{mg}$ in patients $<75 \mathrm{~kg}$ ) can be added (B1).

4) A daily fixed-dose combination of paritaprevir $(150 \mathrm{mg}) /$ ritonavir (100 mg)/ombitasvir ( $25 \mathrm{mg}$ ) plus twice-daily dosed dasabuvir $(250 \mathrm{mg}$ ) and weight-based ribavirin $(1,200 \mathrm{mg}$ in patients $\geq 75 \mathrm{~kg}, 1,000 \mathrm{mg}$ in patients $<75$ $\mathrm{kg}$ ) for $\mathbf{2 4}$ weeks is recommended for post-liver transplantation patients infected with HCV genotypes 1, who have early stage fibrosis (Metavir stage F0-F2) (B1).

5) A daily fixed-dose combination of paritaprevir $(150 \mathrm{mg}) /$ ritonavir (100 mg)/ombitasvir (25 mg) and weight-based ribavirin (1,200 mg in patients $\geq 75 \mathrm{~kg}, 1,000 \mathrm{mg}$ in patients $<75 \mathrm{~kg}$ ) for 12-24 weeks can be used for post-liver transplantation patients infected with HCV genotype 4 (C1).

4. Treatment of patients who develop recurrent HCV infection after liver transplantation: HCV genotype 2

1) Daily sofosbuvir (400 mg) and weight-based ribavirin $(1,200 \mathrm{mg}$ in patients $\geq 75 \mathrm{~kg}, 1,000 \mathrm{mg}$ in patients $<75$ $\mathrm{kg}$ ) for 12-24 weeks can be used for post-liver transplantation patients infected with HCV genotype 2 (C1).

2) Daily daclatasvir (60 mg), sofosbuvir (400 mg), and low initial dose of ribavirin (600 $\mathrm{mg}$, increased as tolerated) for 12 weeks can be used for post-liver transplantation patients infected with HCV genotype 2 (C1). Daily daclatasvir (60 mg) and sofosbuvir (400 mg) for 24 weeks can be used for patients who are ribavirin intolerant or ineligible (C1).

5. Treatment of patients who develop recurrent HCV infection after liver transplantation: HCV genotype 3

1) Daily daclatasvir (60 mg), sofosbuvir (400 mg), and a low initial dose of ribavirin ( $600 \mathrm{mg}$, increased as tolerated) 
for 12 weeks can be used for post-liver transplantation patients infected with HCV genotype 3 (C1). Daily daclatasvir (60 mg) and sofosbuvir (400 mg) for 24 weeks can be used for patients who are ribavirin intolerant or ineligible (C1).

2) Daily sofosbuvir (400 mg) and weight-based ribavirin $(1,200 \mathrm{mg}$ in patients $\geq 75 \mathrm{~kg}, 1,000 \mathrm{mg}$ in patients $<75$ $\mathrm{kg}$ ) for 24-48 weeks can be used for patients who are ribavirin intolerant or ineligible (C1).

6. When DAAs are administered to patients after transplantation, drug-drug interactions with immunosuppressants should be considered. The concomitant use of simeprevir and cyclosporine $A$ is not recommended in this setting (A2). When using the combination of ritonavir-boosted paritaprevir, ombitasvir and dasabuvir, tacrolimus and cyclosporine dose must be adjusted, and the use of mTOR inhibitors is not recommended ( $A 2)$.

7. Interferon-containing treatment for HCV reinfection after liver transplantation is recommended with histological confirmation of chronic hepatitis C (B2). The treatment regimen could be either combination therapy with PegIFN- $\alpha$ plus ribavirin or monotherapy with PegIFN- $\alpha$ (B2). Liver biopsy is often required to differentiate causes of liver function deterioration during antiviral treatment (C1).

8. When antiviral therapy is considered in non-hepatic solid organ transplant recipients, DAA therapy is preferred over interferon-containing therapy (C1).

\section{TREATMENT OF ACUTE HEPATITIS C}

Acute hepatitis $C$ is defined as hepatitis C presenting within 6 months of the exposure. The spontaneous recovery rate of acute hepatitis C varies from 20-50\%. ${ }^{74,82,373-375}$ Before the DAA era, although treatment could be initiated immediately after the diagnosis of acute hepatitis $C$, evidence supports a therapeutic strategy of delaying treatment for 8-12 weeks to allow spontaneous remission. ${ }^{74,376,377}$ According to a randomized controlled study comparing immediate with delayed treatment for 12 weeks, the SVR rate of the delayed treatment was not inferior to that of the immediate treatment in terms of the spontaneous recovery rate and treatment-induced SVR. ${ }^{378}$ Nevertheless, diagnosis of acute hepatitis C has not always been straightforward. Anti-HCV antibody starts to appear at the time of highest ALT and when the blood HCV RNA level is declining, which is $\sim 8-12$ weeks after infection when most patients do not show specific symptoms. ${ }^{140}$ Therefore, testing for serum HCV RNA is useful for diagnosis and treatment when acute hepatitis C is suspected but anti-HCV antibody is negative. The SVR rate can reach $80-90 \%$ when acute hepatitis C is treated with conventional interferon alpha or by PegIFN- $\alpha$ monotherapy for 24 weeks. ${ }^{379-385}$ PegIFN- $\alpha 2 b$ and ribavirin combination therapy did not increase the SVR rate compared to that of PegIFN- $\alpha 2 b$ monotherapy. ${ }^{378,381}$ No clear additional benefit of combining ribavirin with interferon alpha or PegIFN- $\alpha$ is apparent to date.

The optimal treatment duration for acute hepatitis $C$ remains unclear. A randomized controlled study ( $n=34$ per group) reported no significant difference in SVR rates $(82.4 \%$ in the 12 -week treatment group and $91.2 \%$ in the 24 -week treatment group) irrespective of HCV genotypes. ${ }^{385}$ In addition, studies reporting good therapeutic outcomes of acute hepatitis $C$ have tended to adopt a 24-week treatment. ${ }^{379,380,384-386}$ However, a recently published randomized controlled study of 120 patients that compared 12 weeks of PeglFN- $\alpha$ monotherapy, 12 weeks of PeglFN- $\alpha$ and ribavirin combination therapy and 24 weeks of PegIFN- $\alpha$ monotherapy reported no significant difference in SVR rates, ${ }^{387}$ and recommended 12 weeks of PegIFN- $\alpha$ monotherapy.

Data on neither the efficacy nor the cost-effectiveness of treating acute hepatitis $C$ with DAAs are available. However, currently available DAA-containing treatments for chronic $\mathrm{HCV}$, which are highly effective with very few adverse effects, markedly reduce the advantage of treating HCV at the acute stage. Therefore, persons with acute HCV infection can be monitored for $\geq 6$ months for spontaneous HCV clearance and then treated according to the recommendations for chronic infection if indicated. In cases in which a DAA-containing regimen is recommended and deferring treatment would be a threat to the patient's health, immediate treatment can be initiated using the same recommendations as for chronic infection.

\section{[Recommendations]}

1. When the treatment is started at the acute stage, initiation of treatment can be postponed for 8-12 weeks after onset of acute hepatitis $C$ to allow spontaneous recovery (A2).

2. Patients with acute hepatitis $C$ can be treated with PegIFN monotherapy for 12 weeks (A2).

3. If a delay in treatment initiation is acceptable, spontaneous HCV clearance can be monitored for at least 6 months (C1). 4. Treatment after the 6-month monitoring should follow the recommendations for chronic infection (C1). 
The Korean Association for the Study of the Liver (KASL) KASL clinical practice guidelines: Management of Hepatitis C

\section{MONITORING AND MANAGEMENT OF AD- VERSE EFFECTS DURING ANTIVIRAL TREAT- MENT FOR HCV INFECTION}

\section{Monitoring during and after antiviral treatment}

Poor adherence to therapies increases the rate of treatment failure due to virologic breakthrough, relapse after treatment, occurrence of RAV, etc. Therefore, patients should be educated prior to antiviral treatment regarding the importance of compliance and adverse reactions to the antiviral drugs. During the period of antiviral treatment, efforts should be made to increase adherence through regular follow-up visits for monitoring and management of side effects. Because excessive drinking has an impact on treatment adherence, ${ }^{388}$ patients should be recommended to stop or reduce alcohol consumption before and during the treatment. Also, DAAs have potential drug-drug interactions with clinically relevant drugs used for underlying concurrent co-morbid diseases of patients with chronic hepatitis C. Therefore, clinicians should advise patients on potential drug-drug interactions.

To estimate the effect of DAA-containing regimens as antiviral treatments for chronic hepatitis $C$, virologic response is assessed by measuring HCV RNA levels at weeks 4, 8, and 12-24 of treatment or at the end of treatment, and 12 or 24 weeks after treatment. To evaluate each individual therapeutic response and to set the treatment duration of the dual combination of peginterferon alpha and ribavirin, virologic responses are assessed by measuring HCV RNA levels at week 4, 12, and 24 of treatment or at the end of treatment, and 24 weeks after treatment, depending on the HCV genotype.

Stopping rules have been defined for combination therapy with simeprevir, peginterferon alpha and ribavirin; if the HCV RNA value is higher than $25 \mathrm{IU} / \mathrm{mL}$ at week 4 of treatment with good compliance, treatment should be stopped. ${ }^{290,291}$ Also, with combination therapy with sofosbuvir, peginterferon alpha and ribavirin for 12 weeks, if HCV RNA is detected at week 4 of treatment with good compliance, treatment may be stopped. ${ }^{259,389-391}$ There are no clear criteria for stopping treatment with IFN-free DAA regimens, but if the HCV RNA level exceeds $25 \mathrm{IU} / \mathrm{mL}$ in week 4 of treatment, it should be tested again in week 6 . If at that time the HCV RNA concentration is $\geq 10$-fold higher than that in week 4 , treatment should be stopped. ${ }^{124,392}$ If HCV RNA is not elevated but remains detectable at week 6 or 8 of treatment, treatment may be stopped. ${ }^{124,392}$ If HCV RNA is not detected at week 4 of treatment, virologic breakthrough can occur at weeks 4-12 of treat- ment; therefore, HCV RNA should be measured at week 8 of treatment and if is detected or elevated, and compliance is good, treatment should be stopped. ${ }^{233}$

Continuous observation of undetectable HCV RNA after achieving an SVR can be regarded as complete eradication of HCV. HCV re-infection is possible even after achieving an SVR, mainly in PWID. ${ }^{393-397}$ Therefore, follow-up is needed to assess re-infection or relapse of HCV after achieving an SVR. A risk of HCC remained even after achieving an SVR in cases with accompanying cirrhosis or advanced hepatic fibrosis prior to treatment. ${ }^{241}$ In these patients, monitoring for HCC according to the surveillance strategy and management of general complications of cirrhosis are needed. If an SVR is not achieved, the incidence of HCC and disease progression are significantly higher compared to cases with an SVR, ${ }^{241,393}$ and continuous management of chronic hepatitis is necessary in cases without an SVR.

\section{[Recommendations]}

1. The patient should be informed before treatment that adherence is important for achieving an SVR. The patient's adherence should be checked regularly during the treatment. Also drug- drug interactions should be checked upon prescription of a new drug for a comorbid and underlying disease (A1).

2. Serum HCV RNA levels should be measured at weeks 4, 8, and 12-24 of treatment or at the end of treatment depending on the regimen (B1). If HCV RNA is detected at week 4 of DAA treatment (HCV RNA $>25 \mathrm{IU} / \mathrm{mL}$ ), it should be retested at week 6 and if it is elevated by greater than 10 -fold at week 6 , discontinuation of treatment should be considered (C1).

3. To evaluate each individual's therapeutic response and to modify the duration of treatment during PegIFN- $\alpha$ and ribavirin combination therapy, serum HCV RNA assays should be performed at weeks 4,12 , and 24 of treatment or at the end of treatment, depending on the HCV genotype (B1). In cases of genotype 1, treatment should be stopped in patients who fail to achieve an EVR (A1). Patients who achieve a CEVR can be treated for 48 weeks (A1). Patients with a pEVR should be re-tested at week 24; if HCV RNA remains positive, treatment should be stopped (A1).

4. HCV RNA should be measured at 12 or 24 weeks after the cessation of treatment to evaluate therapeutic effects and to identify the achievement of an SVR (A1). 
Table 13. Adverse events of pegylated interferon- $\alpha$ (or interferon- $\alpha$ ) and ribavirin

\begin{tabular}{lll}
\hline Possible related drug & \multicolumn{1}{l}{ Side effects } \\
\hline $\begin{array}{l}\text { Pegylatedinterferon-alpha } \\
\text { or Interferon-alpha }\end{array}$ & Flu-like symptoms & Fatigue, Headache, Fever, Myalgia, Arthralgia \\
\hline & Bone marrow suppression & Neutropenia, Thrombocytopenia \\
\hline & Autoimmune diseases & Depression, Irritability, Insomnia, Apathy \\
& Hashimoto thyroiditis, Graves' disease \\
\hline Gastrointestinal & SLE, Type 1 DM, Bronchial asthma \\
\hline Dermatologic & Pulmonary fibrosis, Interstitial pneumonitis \\
\hline Ophthalmologic & Nausea, Anorexia, Dyspepsia, Diarrhea \\
\hline Others & Alopecia, Rash, Dry skin, Skin itching, Psoriasis \\
\hline & Dry eye, Dry mouth, Stomatitis, Reaction at injection site \\
\hline
\end{tabular}

SLE, Systemic lupus erythematosus; DM, Diabetes Mallitus.

5. Continuous undetectable HCV RNA after achieving an SVR can be regarded as complete eradication of HCV (C1).

6. Risks of hepatocellular carcinoma and complications of chronic liver disease remain after achieving an SVR in patients with cirrhosis or advanced hepatic fibrosis, and continuous management and surveillance following the strategies for chronic liver disease are needed (B1).

7. If an SVR is not achieved, continuous management of chronic liver disease is necessary (B1).

\section{Adverse effects of antiviral therapy and their management}

A thorough education about the process of treatment, and expected adverse reactions and their management encourages patients to continue treatment. Adverse reactions should be assessed at week 2-4 of treatment, and progress should be monitored at about 4-12-week intervals. DAA has fewer adverse reactions and tends to be well tolerated. Commonly reported adverse reactions are fatigue, headache, nausea, etc.; however, less than $1 \%$ of patients stop treatment due to adverse reactions. Adverse reactions of each drug are described in detail in the section 'New drugs, direct-acting antivirals (DAA)'. A liver function test is performed within 4 weeks of treatment, and stopping of treatment may be considered when ALT is over 10-fold higher than the upper limit of the normal range, or when there is a risk of acute liver failure, such as an elevated bilirubin level or extended pro- thrombin time, even if the elevation of ALT is insignificant.

More than $20 \%$ of patients treated with PegIFN- $\alpha$ and ribavirin combination therapy experience headache, fever, myalgia, muscular rigidity, arthralgia, nausea, anorexia, weight loss, diarrhea, hair loss, skin rash, pruritus, inflammation at sites of injection, dyspnea, fatigue, insomnia, irritability, or depression (Table 13). ${ }^{254,261,262,398}$ However, the severity and/or frequency of these adverse effects may vary, as these were reported by patients enrolled in clinical trials. ${ }^{398}$

Adverse effects after PegIFN- $\alpha$ injection can be classified as flulike symptoms, myelosuppression, neuropsychological problems, and autoimmune dysfunction. Flu-like symptoms including fever, fatigue, myalgia, or nausea occur in $\sim 37 \%$ of patients, $254,261,262,398$ but these symptoms can be alleviated by administration of analgesics and usually improve 4-6 weeks after treatment. ${ }^{398}$ Myelosuppression causes neutropenia and thrombocytopenia, the main causes of dose reduction, and often set the therapeutic limit in patients with cirrhosis. Dose of PegIFN- $\alpha$ should be reduced or skipped in cases of severe adverse effects. Especially, when the absolute neutrophil count decreases to $<750 / \mathrm{mm}^{3}$ or platelet count decreases to $<50,000 / \mathrm{mm}^{3}$, dose reduction should be considered; when the absolute neutrophil count decreases to $<500 /$ $\mathrm{mm}^{3}$ or platelet count decreases to $<25,000 / \mathrm{mm}^{3}$, drug discontinuation should be considered. Later, re-administration of the drugs can be considered following adequate recovery of absolute neutrophil count and platelet count; for example, 50\% of the previous dose can be administered if the absolute neutrophil count recovers to $\geq 1,000 / \mathrm{mm}^{3}$, and platelet count to $\geq 75,000 / \mathrm{mm}^{3}$, with 
The Korean Association for the Study of the Liver (KASL) KASL clinical practice guidelines: Management of Hepatitis C

continuous monitoring of these cell counts. Although evidence for the role of granulocyte colony stimulating factor (G-CSF) in terms of reducing the infection rate and improving SVR is insufficient, use of G-CSF can be considered in some patients with cirrhosis. ${ }^{399}$ Treatment should be halted in cases of acute deterioration of hepatitis with elevation of ALT to over 10-fold the upper-normal level or severe bacterial infection, such as sepsis. There is a report that thrombopoietin receptor agonist can raise platelet count in cirrhosis prior to treatment. ${ }^{400}$ However, this drug should be used very carefully, since evidence for improvement of the SVR rate by this drug remains insufficient, whereas it has a risk of portal vein thrombosis. $^{401}$

Neuropsychological problems including insomnia, difficulty in concentrating, memory impairment, irritability, or apathy can be caused by PegIFN- $\alpha$. Especially, severe depression can provoke a suicide attempt, and so careful observation is required during antiviral treatment. ${ }^{402}$ Past history of depression should be checked, since uncontrolled depression is a contraindication to treatment. Depression occurs in about $28 \%$ of patients during treatment, ${ }^{403}$ and antidepressants such as serotonin uptake inhibitors can be used to maintain the treatment. ${ }^{404}$ Preventive administration of antidepressants may reduce the occurrence of depression during treatment although there is a lack of evidence that this would increase the SVR rate. ${ }^{403-405}$

Thyroid complications can occur in $\sim 15-20 \%$ of patients, due to immunomodulatory function of PegIFN- $\alpha,{ }^{398,406}$ which may be due to autoimmune or non-autoimmune causes; autoimmune thyroid diseases are classified as Graves' disease, Hashimoto's disease, and auto-antibody generation against the thyroid gland ${ }^{407}$ and non-autoimmune thyroid disease results from thyroid damage mediated by HCV itself. ${ }^{406-408}$ Hashimoto's disease is the most common; it begins with hyperthyroidism and may progress to hypothyroidism. Thyroid function may not recover after the cessation of treatment. ${ }^{409-411}$ Discontinuation of treatment should be considered in cases of severe hyperthyroidism during interferon administration, while treatment can be maintained with careful observation if hyperthyroidism is not severe. ${ }^{412}$ In cases of hypothyroidism at the beginning, interferon therapy can be maintained by administering thyroxine. ${ }^{406}$ Meanwhile, thyroid gland dysfunction can occur even after the end of treatment ${ }^{398}$ and thyroid-stimulating hormone (TSH) and free thyroxine levels should be assessed at 2-4-month intervals during treatment and regularly for 1 year after the termination of treatment.

Various autoimmune diseases, such as systemic lupus erythematosus, type 1 diabetes mellitus, asthma, interstitial pulmonary fibrosis, or thyroid diseases, can be induced by interferon therapy. ${ }^{413}$ Therefore, baseline evaluation of these diseases is necessary prior to the initiation of treatment, although their presence is not an absolute contraindication to treatment especially, particularly if they are well controlled. ${ }^{398,406}$ Other adverse effects related to PegIFN- $\alpha$, such as visual-field defect, retinal hemorrhage and edema, hearing defect, tinnitus, vomiting, nausea, pruritus, weight loss and hair loss, improve after termination of treatment. ${ }^{398}$ The frequency of retinal defect is $\sim 3.8-30.9 \%{ }^{414-418}$ and the clinical course varies from severe visual field defect to no symptoms. The retina should be checked prior to treatment in cases with risk factors such as old age, hypertension, or diabetes ${ }^{418-420}$ despite the fact that pretreatment and regular follow-up evaluation of the retina remains debatable. Hearing loss occurs in $<1 \%$ of patients and it cannot be recovered completely even after termination of treatment. ${ }^{421}$

A common adverse effect of ribavirin is hemolytic anemia due to dose-dependent direct toxicity to erythrocytes; this can be a barrier to successful treatment. ${ }^{422}$ Anemia due to ribavirin can worsen existing ischemic heart or pulmonary diseases. ${ }^{422}$ Immediate dose reduction by $200 \mathrm{mg}$ should be considered if the hemoglobin level decreases to $<10 \mathrm{~g} / \mathrm{dL}$ and drug discontinuation should be considered in cases with $<8.5 \mathrm{~g} / \mathrm{dL}$ hemoglobin. Re-administration of the reduced dose is possible when anemia improves. Recombinant erythropoietin can be used in cases of severe anemia in order to prevent dose reduction or not to stop ribavirin, although the evidence for erythropoietin increasing the SVR rate is lacking. ${ }^{399,423}$ Meanwhile, ribavirin can cause congenital deformity during pregnancy, therefore thorough contraception is essential during treatment and for 6 months after treatment for both male and female patients. ${ }^{424}$ Other adverse effects related to ribavirin include fatigue, pruritus, rashes, sinusitis, and gout.

Educating patients on treatment-related adverse effects and their management facilitates maintenance of the therapy. Detection of adverse reactions during the first 2-4 weeks of the treatment is important, and monitoring at 4-12-week intervals thereafter is required even if the patients are tolerating the antiviral therapy.

\section{[Recommendations]}

1. Attention should be paid to the adverse reactions of individual drugs in DAA regimens, and interactions with concurrent drugs for underlying diseases should be monitored (A1).

2. For management of adverse reactions to PegIFN- $\alpha$ and riba- 
virin combination therapy, the following are recommended:

1) Pretreatment evaluation of depression, cardiac and pulmonary diseases, hypertension, diabetes mellitus, thyroid diseases, or anemia is required to monitor adverse effects of treatment (B1).

2) Monitoring of adverse effects at 2-4-week intervals after treatment initiation and thereafter at 4-12-week intervals during the treatment (C1).

3) If the absolute neutrophil count decreases to $<750 / \mathrm{mm}^{3}$ or platelet count decreases to $<50,000 / \mathrm{mm}^{3}$, dose reduction of PegIFN- $\alpha$ should be considered; if the absolute neutrophil count decreases to $<500 / \mathrm{mm}^{3}$ or platelet count decreases to $<25,000 / \mathrm{mm}^{3}$, discontinuation of PegIFN- $\alpha$ should be considered. Later, re-administration of PegIFN- $\alpha$ at a reduced dose can be considered following adequate recovery of absolute neutrophil count and platelet count; those counts should be monitored continuously (C2).

4) Dose reduction of ribavirin should be considered when anemia with hemoglobin level $<10 \mathrm{~g} / \mathrm{dL}$ occurs and discontinuation of ribavirin should be considered in cases of a hemoglobin level of $<8.5 \mathrm{~g} / \mathrm{dL}$. Later, when anemia improves, re-administration of ribavirin at a reduced dose is possible, and the hemoglobin level should be monitored continuously (C2).

5) Monitoring of TSH and free thyroxine levels at 2-4-month intervals is recommended to detect thyroid abnormalities (C1).

6) Appropriate management is needed when depression develops during antiviral treatment, and antiviral treatment should be halted in cases of severe depression (C1).

7) Regardless of sex, under treatment that includes ribavirin, contraception is essential during treatment and for 6 months thereafter (A1).

\section{TREATMENT OF SPECIAL POPULATIONS}

Because clinical trials on patients in specific medical conditions have many limitations, individualized antiviral treatment should be used in these populations.

\section{Person who inject drugs}

Injection drug abuse is the main route of HCV transmission, and the abusers show a significantly higher HCV infection rate compared to those without a history of drug abuse. ${ }^{37,425,426}$ Anti-HCV positive rate of Korean intravenous drug users has been reported to be $48.4-79.2 \%$. $^{23-25,427}$

Psychotropic agents (ecstasy and methamphetamine), cannabis, and narcotics are major causes of drug crimes, and psychotropic medicine, the proportion of which increased steadily after 2011, accounted for $81.3 \%$ according to the Annual Narcotics Crime White Paper published by the Supreme Prosecutor's Office (SPO). Treatment of persons who inject drugs (PWID) with chronic HCV infection makes a significant contribution to reducing liver-related complications and transmission to other healthy persons. However, active PWID tends to have an increased likelihood of treatment failure and reinfection if they do not receive adequate support for drug abuse. For this reason, multidisciplinary cooperative treatment among medical and psychiatric counseling services and social support resulted in a significant increase in adherence during therapy.

A meta-analysis including over 2,800 injection drug users showed SVR rates of $44.9 \%$ in HCV genotype 1 and $70.0 \%$ in HCV genotype 2 and 3 patients treated with PegIFN- $\alpha$ and ribavirin. ${ }^{428}$ DAA-based safety and treatment outcomes have not been fully evaluated; however, drug-drug interaction studies reported no clinically important interactions between sofosbuvir and simeprevir, methadone, buprenorphine ${ }^{429,430}$ and daclatasvir, ${ }^{431,432}$ ombitasvir/paritaprevir/ritonavir with dasabuvir and methadone, and buprenorphine. ${ }^{433,434}$ In addition to opioid substitution therapy, antidepressants, antipsychotics and sedatives are frequently used in patients or used by patients with addiction problems, and no significant drug-drug interactions with sofosbuvir has been reported. Simeprevir increases the blood concentration of midazolam after oral administration, and possibly also triazolam. Little data are available for daclatasvir. ${ }^{429}$

\section{[Recommendations]}

1. PWID should be treated following the treatment rules for persons without drug abuse after managing drug-drug interactions (B1).

2. Multidisciplinary cooperative treatment among medical and psychiatric counseling services, social support by specialists on drug abuse and improvement of social environment can increase compliance with treatment in intravenous drug users (B2).

\section{Chronic kidney disease}

HCV infection rates in dialysis patients differ among regions 
The Korean Association for the Study of the Liver (KASL) KASL clinical practice guidelines: Management of Hepatitis C

from $3 \%$ to $80 \%,{ }^{435}$ anti-HCV positivity rates from the late 1990 s to early 2000s in Korea were 5.9-14.7\%..$^{27,28,436}$ In contrast, the Dialysis Registry, ESRD Registry Committee of Korean Society of Nephrology reported a rate of $2.2 \%$ in $2014 .^{29}$

The HCV infection rate is high in chronic kidney disease patients. However, anti-HCV screening may not be needed for these patients. Screening should be selectively conducted when HCVrelated glomerulonephritis clinically presenting as hematuria, albuminuria, or cryoglobulinemia is suspected. However, anti-HCV should be tested in patients undergoing maintenance dialysis for the first time or who were transferred from other dialysis units. In addition, when unexplained abnormal liver-related biochemical tests is found or HCV exposure is suspected, anti-HCV antibodies should be tested and patients who are contiguously anti-HCVnegative should undergo HCV RNA assays. ${ }^{245,437}$ The optimal interval for surveillance of HCV infection in anti-HCV negative patients in dialysis units is 6-12 months, taking into consideration the HCV infection rate of the dialysis unit in question.

Dialysis patients infected with HCV show a higher mortality rate and more rapid progression to cirrhosis or hepatocellular carcinoma compared to non-dialysis patients. ${ }^{438-440}$ Patients scheduled for kidney transplantation should receive an anti-HCV assay and consider HCV treatment because the survival rate after kidney transplantation tends to be low with a possibility of graft rejection and increased occurrence of diabetes and membranous nephritis compared with patients without HCV. ${ }^{429}$ Interferon-based antiviral therapy is not recommended after kidney transplantation due to possible graft rejection; ${ }^{441-447}$ however, DAA-based antiviral therapy can be safely applied after kidney transplantation.

Indications for HCV treatment in chronic kidney disease patients should be determined considering liver disease condition and therapeutic complications. Simeprevir, asunaprevir, daclatasvir, ledipasvir, sofosbuvir and ombitasvir/paritaprevir/ritonavir with dasabuvir are administered at the same dose as in those without kidney disease in patients with mild to moderate renal impairment (creatinine clearance $30-80 \mathrm{~mL} / \mathrm{min}$ ). Safety and efficacy are not fully evaluated in patients with creatinine clearance $(\mathrm{CrCl})<30$ $\mathrm{mL} / \mathrm{min}$, and asunaprevir should be applied at $100 \mathrm{mg}$ daily in non-dialysis patients with $<30 \mathrm{~mL} / \mathrm{min}$. For non-cirrhotic patients with $\mathrm{CrCl}<30 \mathrm{~mL} / \mathrm{min}$ for whom the urgency to treat (or retreat) is high and renal transplant is not an immediate option, a daily fixed-dose combination of paritaprevir (150 mg)/ritonavir (100 $\mathrm{mg}$ )/ombitasvir (25 mg) with twice-daily dosed dasabuvir (250 $\mathrm{mg}$ ) (for HCV genotype $1 \mathrm{~b}$ infection) is recommended. ${ }^{448}$ For patients with $\mathrm{HCV}$ genotypes 2 and 3 infection and $\mathrm{CrCl}<30 \mathrm{~mL} /$ min, PEG-IFN and dose-adjusted ribavirin is recommended. However, this recommendation is based on limited safety and efficacy data. Dose adjustment is needed depending on the severity of kidney disease, since PegIFN- $\alpha$ and ribavirin clearance is reduced according to the degree of impaired kidney function. Moreover, ribavirin should be carefully used in patients with creatinine clearance under $50 \mathrm{~mL} / \mathrm{min}$ since ribavirin can cause severe hemolytic anemia. ${ }^{449}$ Patients with mild kidney disease (glomerular filtration rate (GFR) $\geq 60 \mathrm{~mL} / \mathrm{min}$ ) can be administered the same doses of therapeutic drugs as those without kidney disease. If the patient has severe kidney disease (GFR of $15-59 \mathrm{~mL} / \mathrm{min}$ ), $135 \mu \mathrm{g}$ of PegIFN- $\alpha-2$ a or $1 \mu \mathrm{g} / \mathrm{kg}$ of PegIFN- $\alpha-2 b$ along with 200-800 $\mathrm{mg} /$ day of ribavirin twice a day with a gradual increase in dose is recommended. ${ }^{436}$ Patients on dialysis can take either interferon alpha or PegIFN- $\alpha$, although combination with ribavirin is not recommended. The SVR rates varied from $7-97 \%$ in a study of combination therapy with PegIFN- $\alpha(135 \mu \mathrm{g} /$ week) and low-dose ribavirin (200 mg/day) in patients on dialysis, and most studies reported a high rate of treatment discontinuation. Antiviral therapy for HCV can be conducted in patients with HCV-related cryoglobulinemia or membranous glomerulonephritis. Immunosuppressive therapy or plasma exchange can be performed prior to antiviral treatment in such patients with nephrotic syndrome or a rapid decrease in kidney function. ${ }^{450-452}$

\section{Recommendations}

1. Testing for anti-HCV should be performed in patients with chronic kidney disease who is under or planning to undergo kidney replacement therapy such as dialysis or kidney transplantation (B1).

2. HCV RNA should be tested to confirm HCV infection in patients with idiopathic liver disease despite negative anti$\mathrm{HCV}$ results or in anti-HCV-positive patients (B1).

3. Simeprevir, asunaprevir, daclatasvir, ledipasvir, sofosbuvir and ombitasvir/paritaprevir/ritonavir with dasabuvir can be used without dose adjustment in patients with mild to moderate renal impairment (creatinine clearance $30-80 \mathrm{~mL} / \mathrm{min}$ ) (B1).

4. Safety and efficacy data of DAA-based antiviral therapy are not available in patients with $\mathrm{CrCl}<30 \mathrm{~mL} / \mathrm{min}$ (B1).

5. Combination therapy using PegIFN- $\alpha$ ( $135 \mu \mathrm{g}$ of alpha-2a or $1 \mu \mathrm{g} / \mathrm{kg}$ alpha-2b) and ribavirin (200-800 mg/day) or interferon alpha and ribavirin can be used in chronic hepatitis $C$ patients with severe kidney disease not undergoing hemodialysis (15-59 mL/min glomerular filtration rate) (C2). 
Table 14. Concomitant Use of HIV and HCV drugs*

HIV drugs $\begin{gathered}\text { SCV drugs } \\ \text { Sofosbuvir } \begin{array}{c}\text { Ledipasvir/ } \\ \text { Sofosbuvir }\end{array} \text { Daclatasvir Asunaprevir Simeprevir } \begin{array}{c}\text { Ombitasvir/ } \\ \text { Paritaprevir/ } \\ \text { Ritonavir } \\ \text { plus Dasabuvir }\end{array}\end{gathered} \begin{gathered}\text { Pegylated } \\ \text { interferon }\end{gathered}$ Ribavirin

NRTIs (Nucleoside analogue reverse transcriptase inhibitor)

$\begin{array}{cccccccccc}\text { 3TC } & 0 & 0 & 0 & \text { NA } & 0 & 0 & 0 & 0 \\ \text { ABC } & 0 & 0 & 0 & \text { NA } & 0 & 0 & 0 & 0 \\ \text { FTC } & 0 & 0 & 0 & \text { NA } & 0 & 0 & 0 & 0 \\ \text { TDF } & 0 & 0 & 0 & \text { NA } & 0 & 0 & 0 & 0 \\ \text { ZDV } & 0 & 0 & 0 & \text { NA } & 0 & 0 & x\end{array}$

Pls (Protease inhibitor)

\begin{tabular}{|c|c|c|c|c|c|c|c|c|}
\hline ATV (unboosted) & 0 & 0 & 0 & $x$ & $x$ & $0^{4)}$ & 0 & 0 \\
\hline ATV/r or ATV/C & 0 & $\mathrm{O}^{2)}$ & O (30 mg) & $x$ & $x$ & $0^{5)}$ & 0 & 0 \\
\hline $\mathrm{DRV} / \mathrm{r}$ or $\mathrm{DRV} / \mathrm{C}$ & 0 & $\mathrm{O}^{2)}$ & 0 & $x$ & $x$ & $x$ & 0 & 0 \\
\hline FPV or FPV/r & 0 & $\mathrm{O}^{2)}$ & 0 & $x$ & $x$ & $x$ & 0 & 0 \\
\hline$L P V / r$ & 0 & $\mathrm{O}^{2)}$ & 0 & $x$ & $x$ & $x$ & 0 & 0 \\
\hline $\mathrm{SQV} / \mathrm{r}$ & 0 & $\mathrm{O}^{2)}$ & 0 & $X$ & $x$ & $x$ & 0 & 0 \\
\hline $\mathrm{TPV} / \mathrm{r}$ & $x$ & $x$ & $x$ & $x$ & $x$ & $x$ & 0 & 0 \\
\hline
\end{tabular}

NNRTIs (Non-nucleoside analogue reverse transcriptase inhibitor)

\begin{tabular}{|c|c|c|c|c|c|c|c|c|}
\hline EFV & 0 & $\mathrm{O}^{3)}$ & $\mathrm{O}(90 \mathrm{mg})$ & $x$ & $x$ & $x$ & 0 & 0 \\
\hline ETR & 0 & 0 & O (90 mg) & $x$ & $x$ & $x$ & 0 & 0 \\
\hline NVP & 0 & 0 & 0 & $x$ & $x$ & $x$ & 0 & 0 \\
\hline RPV & 0 & 0 & 0 & 0 & 0 & $x$ & 0 & $\mathrm{O}$ \\
\hline \multicolumn{9}{|c|}{ INSTIs (Integrase strand transfer inhibitor) } \\
\hline DTG & 0 & 0 & 0 & NA & 0 & 0 & 0 & 0 \\
\hline EVG/C/TDF/TFC & 0 & $x$ & 0 & NA & $x$ & $x$ & 0 & 0 \\
\hline RAL & 0 & 0 & 0 & NA & 0 & 0 & 0 & 0 \\
\hline \multicolumn{9}{|c|}{ CCR5 (Chemokine receptor 5) antagonist } \\
\hline MVC & 0 & 0 & 0 & NA & 0 & $x$ & 0 & 0 \\
\hline
\end{tabular}

*Presenting information is based on the data available until October 2015.

$0=$ HIV drugs that can be used concomitantly.

$X=$ HIV drugs not recommended.

$N A=$ Data not available.

3TC, lamivudine; ABC, abacavir; FTC, emtricitabine; TDF, tenofovir disoproxil fumarate; ZDV, zidovudine; ATV, atazanavir; /r, /ritonavir; /C, /cobicistat; DRV, darunavir; FPV, fosamprenavir; LPV, lopinavir; SQV, saquinavir; TPV, tipranavir; EFV, efavirenz; ETR, etravirine; NVP, nevirapine; RPV, rilpivirine; DTG, dolutegravir; EVG, elvitegravir; RAL, raltegravir; MVC, maraviroc.

1) Monitor for TDF toxicity.

2) If Pl/r (or ATV/c, DRV/c) is used with TDF, increase of TDF concentrations are expected. If co-administration necessary, monitor for TDF-associated toxicities.

3) IF EFV used with TDF/FTC, monitor for TDF toxicity due to increase of TDF concentrations.

4) Reduce ATV dose to $300 \mathrm{mg}$ and take in morning at same time as ombitasvir/pritaprevir/ritonavir plus dasabuvir. If ritonavir cannot be used, choose an alternative HCV regimen.

5) Take ATV 300mg in morning at same time as ombitasvir/paritaprevir/ritonavir plus dasabuvir; discontinue ritonavir or cobicistat in HIV regimen until HCV therapy completed. 
The Korean Association for the Study of the Liver (KASL) KASL clinical practice guidelines: Management of Hepatitis C

\section{Treatment of HCV in patients on dialysis may be considered with either interferon alpha ( $2 a$ or $2 b, 3,000,000$ units, three times a week), or a reduced dose of PegIFN- $\alpha \quad(2 a, 135 \mu \mathrm{g} /$ week or $2 \mathrm{~b}, 1 \mu \mathrm{g} / \mathrm{kg} /$ week) (C2).}

\section{Treatment of patients with HIV or HBV coinfection}

\section{Chronic hepatitis C patients with HIV coinfection}

The HIV and HCV coinfection rate is reported to be $25 \%$ in western countries, ${ }^{30}$ and 5.0-6.6\% in South Korea. ${ }^{31,32,453}$ Since the frequency of coinfection is relatively high, all HIV-infected patients should receive HCV testing, which consists primarily of an anti-HCV assay. However, antibody may not be present in $6 \%$ of HIV-infected patients and an HCV RNA assay should be conducted in patients with idiopathic liver disease who are negative for anti-HCV. ${ }^{454,455}$ Chronic hepatitis C patients with risk factors for HIV infection should be tested for HIV.

HIV-coinfected patients show rapid progress of liver disease, higher incidence of cirrhosis and mortality, and generally higher serum HCV RNA levels compared to those with HCV monoinfection. ${ }^{262,456-462}$ Especially, progression of liver disease tends to accelerate with decreasing CD4+ lymphocyte count and deterioration of immune system. ${ }^{463}$ In contrast, recovery of immune function by antiretroviral therapy can delay the progression of liver disease by reducing HIV-related immune activation and inflammation $^{464-466}$ Therefore, antiretroviral therapy is generally recommended in HIV/HCV-coinfected patients regardless of their CD4+ lymphocyte count. However, antiretroviral therapy should be conducted carefully due to high risk of liver toxicity, especially in HIV/ HCV-coinfected patients with progressed liver disease. ${ }^{467,468}$

Antiretroviral therapy can be delayed in HIV treatment-naïve patients and a CD4+ lymphocyte count $>500 / \mathrm{mm}^{3}$ to avoid drugdrug interactions until HCV treatment is completed. In patients with a CD4 lymphocyte count $<200 / \mathrm{mm}^{3}$, antiretroviral therapy should be initiated promptly, but HCV therapy may be delayed until the patient is stable on HIV treatment. ${ }^{469} \mathrm{HIV} / \mathrm{HCV}$-coinfected patients should be treated identically to HCV-monoinfected patients, and DAA treatment is recommended with priority because of the lower treatment efficacy of interferon-based regimens. To decide on the regimen, considerations include drug-drug interactions with antiretroviral agents (Table 14), prior treatment history and drug tolerance; moreover, expert consultation regarding HIV treatment is recommended. ${ }^{470}$

\section{1) Therapeutic agents}

Simeprevir: Simeprevir can be administered with raltegravir, rilpivirine, maraviroc, enfuvirtide, tenofovir, emtricitabine, lamivudine, abacavir, and dolutegravir; these have no drug-drug interactions. Coadministration with efavirenz, etravirine, nevirapine, cobicistat, or HIV PI is not recommended.

Ribavirin: Anemia related to ribavirin is an increasingly important problem in treatment of HIV coinfection, is more frequent and severe in patients taking zidovudine (AZT), and should be avoided. ${ }^{471}$ Ribavirin can deteriorate didanosine (ddl) toxicity by inhibiting inosine-5-monophosphate dehydrogenase and severe lactic acidosis, steatosis, pancreatitis have been reported in patients taking ddl along with ribavirin; therefore, concomitant use of these two agents is contraindicated. ${ }^{472-474}$ Therefore, patients receiving $A Z T$ and especially ddl should be switched to an equivalent antiretroviral agent before beginning a combination therapy that includes ribavirin.

Asunaprevir: Among antiretroviral agents, $\mathrm{Pl}$ is not recommended because the blood asunaprevir concentration may be increased, and non-nucleoside reverse transcriptase inhibitors (NNRTIs) other than rilpivirine are not recommended because may decrease the therapeutic effect of asunaprevir.

Daclatasvir: Dose adjustment of daclatasvir is not required when used with ritonavir-boosted darunavir or ritonavir-boosted lopinavir. The dose of daclatasvir should be reduced to $30 \mathrm{mg}$ once daily with ritonavir-boosted atazanavir and cobicistat-containing antiretroviral regimens, and an increased dose (90 mg daily) of daclatasvir is recommended when used with efavirenz or etravirine.

Sofosbuvir: No clinically significant drug-drug interactions with most antiretroviral drugs (efavirenz, tenofovir, emtricitabine, rilpivirine, ritonavir-boosted darunavir and raltegravir) have been identified, but coadministration with tipranavir is not recommended.

Ledipasvir and sofosbuvir: When rilpivirine or efavirenz is used with tenofovir as an antiretroviral agent for treatment of HCV-HIV coinfection, tenofovir levels are increased with ledipasvir/sofosbuvir, which may increase the risk of renal toxicity. Concomitant use with ledipasvir/sofosbuvir in patients at high risk for renal toxicity (those with a $\mathrm{CrCl}$ rate $30-60 \mathrm{~mL} / \mathrm{min}$ or pre-existing evidence of Fanconi syndrome) or those taking tenofovir and a ritonavir-boosted $\mathrm{Pl}$, should be monitored for potential renal injury by assessing renal function every 2-4 weeks. ${ }^{392,475}$

Ombitasvir/paritaprevir/ritonavir and dasabuvir: Paritaprevir is an inhibitor of OATP1B1 and can increase indirect bilirubin. Ombitasvir/paritaprevir/ritonavir plus dasabuvir should be 
used with antiretroviral drugs with which they do not have drugdrug interactions; i.e., raltegravir, enfuvirtide, tenofovir, emtricitabine, lamivudine, atazanavir, dolutegravir. As ritonavir has antiHIV activity, HIV/HCV-coinfected patients should have achieved HIV RNA suppression prior to initiation of this regimen due to the potential for low-dose ritonavir to select for HIV PI resistance in patients not undergoing antiretroviral therapy. Especially, because this combination contains $100 \mathrm{mg}$ of ritonavir, the dose of ritonavir used for boosting of HIV protease inhibitors may need to be adjusted (or held) when administered with ombitasvir/paritaprevir/ ritonavir plus dasabuvir and then restored upon completion of HCV treatment. Concomitant use with efavirenz, rilpivirine, darunavir, and ritonavir-boosted lopinavir is not recommended. When this combination is used with ribavirin, unboosted HIV PI, rilpivirine, and efavirenz should not be used. When this combination is used with efavirenz, emtricitabine, tenofovir, gastrointestinal and neurologic adverse events, elevations of ALT can occur. ${ }^{392,476}$

\section{2) Treatment efficacy}

In 50 patients with HCV genotype 1 and HIV co-infection never before treated for HCV and without cirrhosis, the SVR rate was $98 \%$ after 12 weeks of ledipasvir/sofosbuvir treatment. ${ }^{475}$ After 12 weeks of ledipasvir/sofosbuvir treatment in 335 patients coinfected with HIV and genotype 1 or 4 HCV who had been previously treated or untreated for HCV, $20 \%$ of whom were cirrhotic, the SVR rate was $96 \%{ }^{477}$ In 63 patients with HCV genotype 1 and HIV co-infection who were HCV treatment-naïve or had a history of prior treatment failure including cirrhotic patients, the SVR rate was $91-94 \%$ after 12 or 24 weeks of ombitasvir/paritaprevir/ritonavir plus dasabuvir and ribavirin. ${ }^{476}$ After 24 weeks of sofosbuvir plus weight-based ribavirin in 112 treatment-naïve and 114 retreatment genotype $1 \mathrm{HCV}$ coinfected patients including those with compensated cirrhosis, the SVR rates were $85 \%$ and $76 \%$, respectively. ${ }^{348,478}$ After 12 weeks of daclatasvir plus sofosbuvir treatment in 153 previously untreated and treated HIV/HCV coinfected (genotypes 1-4) patients, which included cirrhotic patients, the SVR rate was $97-98 \%{ }^{311}$

In HCV genotype 2 or 3 patients including those with compensated cirrhosis, sofosbuvir plus weight-based ribavirin treatment for 12 weeks in 68 treatment-naïve patients and 24 weeks in 41 retreatment patients resulted in SVR rates of $88 \%$ for genotype 2 and $67 \%$ in genotype $3 \mathrm{HCV}^{478}$ Using the same regimen, $163 \mathrm{HIV}$ coinfected patients (including cirrhotic patients) were treated for 12 weeks (genotype 2 treatment-naïve) or 24 weeks (genotype 3 or 4 and retreated genotype 2 patients); SVR rates were $88 \%$ for geno- type 2, 89\% for genotype 3 and 84\% for genotype $4 \mathrm{HCV}^{348}$

Clinical information regarding sofosbuvir treatment in patients with HIV and HCV genotype 5 and 6 coinfection is limited, and information about retreatment in HIV-coinfected patients, or in simeprevir- or sofosbuvir-experienced patients is also insufficient.

Peginterferon alpha can be used at the same dose recommended for treating HCV monoinfection, and the ribavirin dose can be adjusted depending on body weight (1,000 mg/day for under 75 $\mathrm{kg}, 1,200 \mathrm{mg} /$ day for over $75 \mathrm{~kg}$ ), regardless of HCV genotype in HIV coinfection. ${ }^{479}$ Treatment duration is usually recommended as 48 weeks, regardless of HCV genotype. A shortened duration of therapy to 24 weeks can be effective in genotype 2 and 3 with RVR, and an extended duration of therapy to 60-72 weeks can be helpful in genotype 1 and 4 with pEVR and no RVR. ${ }^{479-483}$

\section{[Recommendations]}

1. All HIV infected patients should be tested for anti-HCV (B1).

2. HCV RNA assays should be conducted in patients with idiopathic liver disease, including those anti-HCV negative and positive (B1).

3. Antiretroviral treatment interruption to allow HCV therapy is not recommended (B1).

4. Interferon-free DAA treatment is recommended as a priority for HIV/HCV-coinfected patients, who should be treated identically HCV-monoinfected patients (B1).

5. When HIV/HCV-coinfected patients are treated with DAAs, drug-drug interactions should be carefully considered and consultation with an HIV treatment expert is recommended if the antiretroviral therapy regimen is to be modified (A1).

6. PegIFN- $\alpha$ can be used at the same dose recommended for treating HCV monoinfection and the ribavirin dose can be adjusted depending on body weight for 48 weeks, regardless of HCV genotype, in HIV-coinfected patients (B2).

\section{Chronic hepatitis C patients with HBV coinfection}

The number of HBV/HCV coinfected patients worldwide is estimated to be $15,000,000,{ }^{484}$ and $2.37 \%$ of anti-HCV positive patients are reported to be coinfected with HBV in South Korea. ${ }^{485}$

A 10-year follow-up study of HCV-monoinfected patients reported an HCC occurrence rate of $28 \%$, whereas HCV/HBV-coinfected patients showed an occurrence rate of $45 \%$, which was significantly higher. ${ }^{486}$ In addition, risks of severe and fulminant hepatitis increase and the incidence of cirrhosis and HCC increas- 
The Korean Association for the Study of the Liver (KASL) KASL clinical practice guidelines: Management of Hepatitis C

Table 15. Concomitant Use of HBV and HCV Drugs*

\begin{tabular}{lcccccccc}
\hline Sofosbuvir & $\begin{array}{c}\text { Ledipasvir/ } \\
\text { Sofosbuvir }\end{array}$ & Daclatasvir & Asunaprevir & Simeprevir & $\begin{array}{c}\text { Ombitasvir/ } \\
\text { Paritaprevir/ } \\
\text { Ritonavir } \\
\text { plus Dasabuvir }\end{array}$ & $\begin{array}{c}\text { Pegylated } \\
\text { Interferon }\end{array}$ \\
Ribavirin
\end{tabular}

"Presenting information is based on the data available until October 2015.

$0=$ No clinical significant interaction expected.

$\triangle=$ Potential interaction, may require close monitoring, alteration of drug dosage or timing of administration.

$X=$ These drugs should not be coadministered.

NA = Data not available.

es in HBV/HCV-coinfected patients compared to those with HBV monoinfection. ${ }^{487-489}$

In patients with HBV/HCV coinfection, blood HCV RNA and HBV DNA levels, which are indicators of the replicative status of each virus, should be evaluated, and if HCV infection is the dominant cause of the liver disease, the same antiviral therapy as for HCV monoinfection is recommended. Indeed, the SVR rate of PegIFN- $\alpha$ and ribavirin is similar to that in HCV monoinfection. ${ }^{490-492}$ Treatment of patients with HBV/HCV coinfection is identical to that of HCV-monoinfected patients. The risk of drug-drug interactions between DAA and anti-HBV agents is low, with the exception of asunaprevir, for which information is lacking. Renal function monitoring is warranted if ledipasvir is used with tenofovir because renal toxicity can be increased (Table 15). Reactivation of HBV is possible during or after HCV treatment, ${ }^{485,493}$ and administration of oral antiviral agents may be indicated if significant proliferation of HBV is confirmed. ${ }^{494}$

\section{[Recommendations]}

1. After confirming the dominant cause of liver diseases in $\mathrm{HBV} / \mathrm{HCV}$ coinfection, treatment following the same rules as HCV monoinfection is recommended, and oral administration of anti-HBV agents may be indicated if significant proliferation of HBV is confirmed (B1).

\section{Hemophilia or thalassemia}

Accompanying HCV infection in patients with hemophilia or thalassemia causes significant increases in morbidity and mortali- ty rates compared to patients without HCV infection. ${ }^{495-499}$ Therefore, aggressive treatment of HCV infection should be considered. Hemophilia A and B caused by deficiency of factor VIII or IX may have increased chance of exposure to HCV due to multiple transfusion caused by spontaneous and traumatic bleeds. Coinfection with HIV/HCV is not a contraindication to liver transplantation in hemophilia, and indications for liver transplantation in patients with hemophilia are the same as those in non-hemophilic individuals. ${ }^{429}$ Therapeutic outcomes in HCV-infected cases with or without hemophilia were similar, and there was no increase in complications regarding bleeding tendency. ${ }^{500,501}$

Severe anemia can occur due to ribavirin in thalassemia, and up to $30-40 \%$ of cases may require blood transfusion at 3-4-week intervals to maintain 9-10 $\mathrm{g} / \mathrm{dL}$ hemoglobin. Therefore careful monitoring is required to confirm hematological complications. However, the frequency of treatment discontinuation and the incidence of other main complications did not increase in these patients. ${ }^{495}$

\section{[Recommendations]}

1. Patients with hemophilia should be treated following the same rules as persons without bleeding disorders (A1).

2. Patients with thalassemia should be treated following the same rules as persons without hemoglobinopathy (B1).

\section{Patients receiving immunosuppressants or cytotoxic chemotherapy}

Although one study defined HCV reactivation as re-emergence of or increase in HCV RNA plus elevation of ALT up to threefold 
the upper limit of normal, ${ }^{502}$ there is no universal consensus on definition of HCV reactivation; blood ALT and HCV RNA levels are commonly used as the criteria.

The incidence of HCV reactivation in patients taking immunosuppressants or under cytotoxic chemotherapy is lower than that of $\mathrm{HBV}^{502-506}$ For example, the reactivation rate of $\mathrm{HCV}$ was $0 \%$ (0/11) compared to $38 \%$ (3/8) for HBV in a study including 98 non-Hodgkin's lymphoma patients receiving chemotherapy. ${ }^{507}$ However, another study of B cell non-Hodgkin's lymphoma reported a higher incidence ( $26.3 \%$ vs. $2.1 \%$ ) of significant elevation of ALT level in HCV-infected patients compared to patients without HCV infection, indicating that HCV reactivation does occur and may cause clinically significant morbidity. ${ }^{508}$

Risk factors for HCV reactivation have not been clearly identified. However, reactivation has been reported to occur more frequently in patients with hematological malignancies. ${ }^{504,509} \mathrm{HCV}$ reactivation has also been reported in patients with solid cancer or who underwent stem cell transplantation. ${ }^{510-513}$ Although death due to $\mathrm{HCV}$ reactivation is rare, ${ }^{514}$ the mortality is similar to that of HBV once severe hepatitis occurs due to HCV reactivation. ${ }^{515-517}$

Strategies to prevent HCV reactivation in these patients have not been established. Conservative therapy and discontinuation of offending drugs are currently recommended. However, one should take into account hepatic morbidity from HCV reactivation and the disadvantages of immunosuppressive drug discontinuation, and decisions should be individualized. Further studies are needed to explore methods of preventing and treating HCV reactivation using DAAs.

\section{Children}

From a Korean study reported in 1996, 2,080 children were recruited and an anti-HCV positive rate was $0.82 \%{ }^{518}$ Transfusion of infected blood components or vertical transmission is the most common cause of HCV infection in children, ${ }^{519}$ although transfusion-related HCV transmission has been reported only rarely after introduction of the screening test in 1991 in South Korea. The global HCV infection rate in pregnant females has been reported to be $0.49-1.7 \%{ }^{16-18}$ A Korean study involving 5,000 pregnant females and another study with 20,000 pregnant females reported antiHCV positivity rates of $0.42-0.44 \%$, where $57-60 \%$ of anti-HCV positive pregnant females were also positive for HCV RNA. ${ }^{19,20}$

The frequency of HCV transmission was reported as 1-6.2\% during the perinatal period, ${ }^{67,69,520,521}$ and the evidence that Cesarean section reduces the risk of vertical HCV transmission is weak. ${ }^{522}$ Although HCV RNA has been detected in human milk, acquisition of HCV infection from human milk has not been documented, and children with chronic HCV infection should not be prevented from attending school or participating in routine activities, including sports. ${ }^{522}$

An anti-HCV assay in children is recommended after 18 months of age, since maternal antibodies can be delivered to newborns. ${ }^{522-524}$ An HCV RNA assay may be performed at 1 or 2 months of age if earlier diagnosis is desired, although the sensitivity is as low as $22 \%$ at that time; therefore, HCV RNA assays should be conducted at the age over 6 months, when the sensitivity reaches $85 \%{ }^{523,525}$

Spontaneous recovery is more frequent in children, and tends to show a normal ALT level, ${ }^{526}$ In addition, HCV infection in children often shows slow progression of hepatic fibrosis and rarely results in severe hepatic damage. However, aggressive treatment instead of waiting until the children reach the age of adult has been suggested, since children usually have a regular life cycle and show higher therapeutic compliance. Aggressive treatment is considered in cases of continuously elevated serum AST/ALT levels or when progressed hepatic fibrosis is confirmed by liver biopsy. In addition, treatment can also be considered when serum AST/ALT is normal or mild fibrosis is detected by liver biopsy since the tools used to predict disease progression are not sufficient in children. ${ }^{527}$

Little data are available in children. ${ }^{528}$ Although in previous studies on HCV-infected children was limited to interferon- $\alpha$ monotherapy due to the potential teratogenic effects of ribavirin, higher SVR rates have recently been reported with the addition of ribavirin to treatment. ${ }^{529-533}$ Therefore, most studies have adapted combination therapy to children, since this approach is standard in adults. Use of PegIFN- $\alpha$ in children over 3 years of age has been approved in North America and Europe. ${ }^{527}$

The dose of PegIFN- $\alpha$ is $60 \mu \mathrm{g} / \mathrm{m}^{2} /$ week for PegIFN- $\alpha 2 b$, and $180 \mu \mathrm{g} / 1.73 \mathrm{~m}^{2} /$ week for PegIFN- $\alpha$ 2a and the dose of ribavirin is $15 \mathrm{mg} / \mathrm{kg}$ twice a day. Genotype 1 and 4 patients should be treated for 48 weeks and genotype 2 and 3 patients should be treated for 24 weeks, similar to adults. ${ }^{527}$ The SVR rate after combination therapy of PegIFN- $\alpha$ and ribavirin (47-53\% in genotype 1 and $80-100 \%$ in genotypes 2 and 3 ) is superior to that of combination therapy with interferon alpha and ribavirin..$^{531-533}$ Factors predictive of an SVR include infection by genotypes 2 and 3, and an HCV RNA titer $<600,000 \mathrm{IU} / \mathrm{mL} .{ }^{529,532,533}$

\section{[Recommendations]}

\section{Diagnosis and evaluation of HCV in children should proceed}


The Korean Association for the Study of the Liver (KASL) KASL clinical practice guidelines: Management of Hepatitis C

following the same rules as in adults (B1).

2. Anti-HCV assay in children is recommended at age $>18$ months since maternal antibodies can be delivered to newborns. If an earlier assay is required, HCV RNA assay may be considered after 6 months of age (B2).

3. HCV infected children aged 3-17 years should be considered appropriate candidates for treatment according to the same criteria used in adults (B1).

4. The dose of PegIFN- $a$ is $60 \mu \mathrm{g} / 1.73 \mathrm{~m}^{2} /$ week for $2 \mathrm{~b}$ and 180 $\mu \mathrm{g} / 1.73 \mathrm{~m}^{2} /$ week for $2 \mathrm{a}$, and the dose of ribavirin is $15 \mathrm{mg} /$ kg/day. Genotype 1 and 4 patients should be treated for 48 weeks and genotype 2 and 3 patients should be treated for 24 weeks (B1).

\section{Conflicts of Interest}

Potential conflicts of interests are as the followings.

Sook-Hyang Jeong: Consulted Gilead, BMS, Abbvie and received grants from BMS, Gilead, Abbvie, MSD, Boehringer Ingelheim, Pharmaking, Kowa.

Jung II Lee: Received grants from MSD, BMS, Gilead, Bayer, Roche, Kowa, Eisai, Novotech, EPS, Eli Lilly, Ildong, Pharmicell, Medigen, received honoraria from BMS, Gilead, and consulted Gilead.

Young Seok Kim: Consulted BMS, Gilead, received honoraria from Gilead, BMS, Bayer, Yuhan, Phillips, and received grants from Gilead, BMS, MSD, Roche, Gambro, Hanmi, Otsuka, Theragen Etex, Bayer, Inventive Health Korea, Bukwang, Ildong, Ferring, Shionogi. Kyung-Ah Kim: Received grants from BMS, Gilead.

Sang Hoon Park: Received honoraria from Yuhan, BMS, Bayer and received grants from Handok, Yuhan, BMS.

Byung Seok Lee: Received honoraria from BMS, Boryung, Roche, MSD, Taejoon, and received grants from Gilead, Yuhan, Boryung, Roche, MSD, BMS, Pharmicell, Ildong, Samil, Bukwang, Dong-A, Handok, Bayer, Chongkundang.

Jun Yong Park: Received honoraria from Yuhan, received grants from BMS, Gilead, Bayer, CJ.

Ki Tae Yoon: Received grants from Gilead, BMS, Roche, MSD, Janssen, Bayer, Boehringer Ingelheim, Abbvie, Arrowhead, Inovio, Eisai, Shionogi, Kowa, Medigen, Gennerex, Sillajen, and received honoraria from Gilead, BMS, Roche, MSD, Bayer, Handok, Boryung, Dong-A, Yuhan, GSK.

Young Kul Jung: Received honoraria from Yuhan, BMS, Boryung, and received grants from Bukwang, Ildong, Handok, BMS, Gilead. Chang Wook Kim: Consulted Gilead, received honoraria from BMS, Daewoong, Roche, Dong-A, Yuhan and received grants from
BMS, Gilead, Yuhan, Handok, Daewoong, Pharmaking, Pharmicell, KT\&G, Roche

Eun Young Cho: Received honoraria from Gilead, BMS, and received grants from Yuhan, Roche.

Geum-Youn Gwak: Received honorarium from Menarini and received grants from Gilead, BMS, MSD, Boehringer Ingelheim, Medigen, Green Cross, Janssen, Eisai, Kowa, Creagen, Bukwang, Green Cross Cell, Otsuka, Dong-A, Bayer.

Woo Jin Chung: Received grants from MSD, BMS, Roche, Bukwang and received honoraria from BMS, Yuhan.

Jeong Han Kim: Consulted MSD.

\section{REFERENCES}

1. Guyatt $G H$, Oxman AD, Vist GE, Kunz R, Falck-Ytter Y, Alonso-Coello $P$, et al. GRADE: an emerging consensus on rating quality of evidence and strength of recommendations. BMJ 2008;336:924-926.

2. Suh DJ, Jeong SH. Current status of hepatitis C virus infection in Korea. Intervirology 2006;49:70-75.

3. Gower E, Estes C, Blach S, Razavi-Shearer K, Razavi H. Global epidemiology and genotype distribution of the hepatitis $C$ virus infection. J Hepatol 2014;61(1 Suppl):S45-S57.

4. Mohd Hanafiah K, Groeger J, Flaxman AD, Wiersma ST. Global epidemiology of hepatitis $C$ virus infection: new estimates of agespecific antibody to HCV seroprevalence. Hepatology 2013;57:13331342.

5. Kim YS, Pai CH, Chi HS, Kim DW, Min YI, Ahn YO. Prevalence of hepatitis $\mathrm{C}$ virus antibody among Korean adults. J Korean Med Sci 1992;7:333-336.

6. Jeong $T H$, Jeon $T H . P C R$ prevalence and risk factors of hepatitis $C$ virus infection in the adult population of Ulsan. J Korean Acad Fam Med 1998;19:364-373

7. Na HY, Park MH, Park KS, Sohn YH, Joo YE, Kim SJ. Geographic characteristics of positivity of anti - HCV and Chonnam province: survey data of 6, 790 health screenees. Korean I Gastroenterol 2001;38:177-184

8. Park KS, Lee YS, Lee SG, Hwang JY, Chung WJ, Cho KB, et al. A study on markers of viral hepatitis in adults living in Daegu and Gyungbuk area. Korean J Gastroenterol 2003;41:473- 479.

9. Seo WT, Lee SS. A study on positive rate of HBs Ag, HBs Ab and antiHCV in Korean adults. Korean J Blood Transfus 1998;9:259-272.

10. Shin HR. Epidemiology of hepatitis C virus in Korea. Intervirology 2006;49:18-22.

11. Kim do Y, Kim IH, Jeong SH, Cho YK, Lee JH, Jin YJ, et al. A nationwide seroepidemiology of hepatitis $C$ virus infection in South Korea. Liver Int 2013:33:586-594. 
12. Kim KA, Jeong SH, Jang ES, Kim YS, Lee YJ, Jung EU, et al. Geographic differences in the epidemiological features of $\mathrm{HCV}$ infection in Korea. Clin Mol Hepatol 2014;20:361-367.

13. Oh HB, Hwang YS, Cho YJ, Kim DS, Kim SI. Experience of anti-HCV antibody immunoblot test in Korean blood donors. Korean J Blood Transfus 1997:8:1-8.

14. Oh DJ, Park YM, Seo YI, Lee JS, Lee JY. Prevalence of hepatitis C virus infections and distribution of hepatitis $C$ virus genotypes among Korean blood donors. Ann Lab Med 2012;32:210-215.

15. Kim MJ, Park Q, Min HK, Kim HO. Residual risk of transfusion-transmitted infection with human immunodeficiency virus, hepatitis $C$ virus, and hepatitis B virus in Korea from 2000 through 2010. BMC Infect Dis 2012;12:160.

16. Moriya T, Sasaki F, Mizui M, Ohno N, Mohri H, Mishiro S, et al. Transmission of hepatitis $C$ virus from mothers to infants: its frequency and risk factors revisited. Biomed Pharmacother 1995;49:59-64.

17. Okamoto M, Nagata I, Murakami J, Kaji S, litsuka T, Hoshika T, et al. Prospective reevaluation of risk factors in mother-to-child transmission of hepatitis C virus: high virus load, vaginal delivery, and negative anti-NS4 antibody. J Infect Dis 2000;182:1511-1514.

18. Claret G, Noguera A, Esteva C, Munoz-Almagro C, Sanchez E, Fortuny $C$. Mother-to-child transmission of hepatitis $C$ virus infection in Barcelona, Spain: a prospective study. Eur J Pediatr 2007;166:12971299.

19. Kim YW, Lee JM, Kim GJ, Lee HM, Kim SY, Lee JS, et al. Hepatitis C virus infection in pregnancy. Korean J Obstet Gynecol 2000;43:597603.

20. Kang MJ, Kim HJ, Park KJ, Kang KH, Ahn HS. Prevalence of HCV infection in pregnant women and vertical transmission. Korean J Obstet Gynecol 2004;47:2045-2050.

21. Lee JM, Lee JM, Yoo HS, Jang UK, Kim DJ, Kim YB, et al. The prevalence of anti-HCV positivity in healthy Korean children. Korean J Hepatol 1996;2:160-165.

22. Kim HS, Choo DH. Prevalence of hepatitis C, B and human immunodeficiency virus among drug users and chronic alcoholic patients in Korea. Korean J Med 1997;52:754-762.

23. Lee SW, Kim SY, Kim JK. Seropositivity of anti-HCV in intravenous drug abusers. J Korean Acad Fam Med 1997;18:1508-1518.

24. Yun H, Kim D, Kim S, Kang S, Jeong S, Cheon Y, et al. High prevalence of HBV and HCV infection among intravenous drug users in Korea. J Med Virol 2008;80:1570-1575.

25. Min JA, Yoon Y, Lee HJ, Choi J, Kwon M, Kim K, et al. Prevalence and associated clinical characteristics of hepatitis $B, C$, and HIV infections among injecting drug users in Korea. J Med Virol 2013;85:575-582.

26. Macias J, Palacios RB, Claro E, Vargas J, Vergara S, Mira JA, et al. High prevalence of hepatitis $C$ virus infection among noninjecting drug users: association with sharing the inhalation implements of crack. Liver Int 2008;28:781-786.

27. Kim H, Kim KT, Yoo JH, Kim BI, Lee SJ, Lee EJ, et al. Prevalence and risk factors of hepatitis $\mathrm{C}$ virus infection in chronic hamodialysis patients(multi-center study). Korean J Med 1997;52:833-840.

28. Shin YH, Kim HK, Choi SD, Kim YS, Shin HS, Won YJ, et al. Prevalence of anti-HCV in hemodialysis patients in Taegu and Kyeongbuk, Korea. Korean J Med 1998;54:640-646.

29. ESRD Registry Committee, Korean Society of Nephrology. Current renal replacement therapy in Korea: Insan memorial dialysis registry, 2014. Kidney Res Clin Pract 2015:117-136.

30. Sherman KE, Rouster SD, Chung RT, Rajicic N. Hepatitis C Virus prevalence among patients infected with Human Immunodeficiency Virus: a cross-sectional analysis of the US adult AIDS Clinical Trials Group. Clin Infect Dis 2002;34:831-837.

31. Kim O, Kim SS, Park MS, Suh SD, Lee MW, Kim KS, et al. Seroprevalence of sexually transmitted viruses in Korean populations including HIV-seropositive individuals. Int J STD AIDS 2003;14:46-49.

32. Lee SH, Kim KH, Lee SG, Chen DH, Jung DS, Moon CS, et al. Trends of mortality and cause of death among HIV-infected patients in Korea, 1990-2011. J Korean Med Sci 2013;28:67-73.

33. Kim SY, Kook JH, Choi IS, Kim SJ, Kook H, Hwang TJ. Viral hepatitis and change of lymphocyte subpopulation in hemophiliacs in Chonnam KwangJu area. Korean J Blood Transfus 2002;13:43-51.

34. Korea Hemophilia Association. 2012 Korean hemophilia annual report. Seoul: Korea Hemophilia Association, 2012.

35. Choi SH. The prevalence of hepatitis C virus infection in leprous patients. Korean J Gastroenterol 1997;30:486-494.

36. Lavanchy D. The global burden of hepatitis C. Liver Int 2009;29 Suppl 1:74-81.

37. Williams IT, Bell BP, Kuhnert W, Alter MJ. Incidence and transmission patterns of acute hepatitis C in the United States, 1982-2006. Arch Intern Med 2011;171:242-248.

38. La Torre G, Gualano MR, Semyonov L, Nicolotti N, Ricciardi W, Boccia A. Hepatitis C Virus infections trends in Italy, 1996-2006. Hepat Mon 2011;11:895-900.

39. Oh HB, Hwang YS, Kim DS, Kim SI, Lee SY, Han KS. Study on the seroincidence of hepatitic $C$ virus infection among blood donors in Korea. Korean J Blood Transfus 1997;8:33-41.

40. Esteban JI, Sauleda S, Quer J. The changing epidemiology of hepatitis C virus infection in Europe. J Hepatol 2008;48:148-162.

41. Rustgi VK. The epidemiology of hepatitis C infection in the United States. J Gastroenterol 2007;42:513-521.

42. Shin HR, Kim JY, Kim JI, Lee DH, Yoo KY, Lee DS, et al. Hepatitis B and $C$ virus prevalence in a rural area of South Korea: the role of acupuncture. Br J Cancer 2002;87:314-318.

43. Cho EJ, Jeong SH, Han BH, Lee SU, Yun BC, Park ET. Hepatitis C virus $(\mathrm{HCV})$ genotypes and the influence of HCV subtype $1 \mathrm{~b}$ on the progression of chronic hepatitis $C$ in Korea: a single center experi- 
The Korean Association for the Study of the Liver (KASL) KASL clinical practice guidelines: Management of Hepatitis C ence. Clin Mol Hepatol 2012;18:219-224.

44. Raimondi S, Bruno S, Mondelli MU, Maisonneuve P. Hepatitis C virus genotype $1 \mathrm{~b}$ as a risk factor for hepatocellular carcinoma development: a meta-analysis. J Hepatol 2009;50:1142-1154.

45. Hnatyszyn HJ. Chronic hepatitis $C$ and genotyping: the clinical significance of determining HCV genotypes. Antivir Ther 2005;10:1-11.

46. Busch MP, Glynn SA, Stramer SL, Strong DM, Caglioti S, Wright DJ, et al. A new strategy for estimating risks of transfusion-transmitted viral infections based on rates of detection of recently infected donors. Transfusion 2005;45:254-264.

47. Vermeulen M, Lelie N, Sykes W, Crookes R, Swanevelder J, Gaggia $L$, et al. Impact of individual-donation nucleic acid testing on risk of human immunodeficiency virus, hepatitis B virus, and hepatitis $C$ virus transmission by blood transfusion in South Africa. Transfusion 2009;49:1115-1125.

48. Shan H, Ren FR, Zhao HY, Zhang YZ, Wen GX, Yao FZ, et al. A multiChinese blood center study testing serologic-negative donor samples for hepatitis C virus and human immunodeficiency virus with nucleic acid testing. Transfusion 2007:47:2011-2016.

49. Papatheodoridis $G$, Hatzakis A. Public health issues of hepatitis $C$ virus infection. Best Pract Res Clin Gastroenterol 2012;26:371-380.

50. Nelson PK, Mathers BM, Cowie B, Hagan H, Des Jarlais D, Horyniak $D$, et al. Global epidemiology of hepatitis $B$ and hepatitis $C$ in people who inject drugs: results of systematic reviews. Lancet 2011;378:571-583.

51. Kermode M. Unsafe injections in low-income country health settings: need for injection safety promotion to prevent the spread of blood-borne viruses. Health Promot Int 2004;19:95-103.

52. Deterding K, Wiegand J, Gruner N, Hahn A, Jackel E, Jung MC, et al. The German Hep-Net acute hepatitis C cohort: impact of viral and host factors on the initial presentation of acute hepatitis $C$ virus infection. Z Gastroenterol 2009;47:531-540.

53. Gutelius B, Perz JF, Parker MM, Hallack R, Stricof R, Clement EJ, et al. Multiple clusters of hepatitis virus infections associated with anesthesia for outpatient endoscopy procedures. Gastroenterology 2010;139:163-170

54. Hayes MO, Harkness GA. Body piercing as a risk factor for viral hepatitis: an integrative research review. Am J Infect Control 2001;29:271-274.

55. Ernst E, Sherman KJ. Is acupuncture a risk factor for hepatitis? Systematic review of epidemiological studies. J Gastroenterol Hepatol 2003;18:1231-1236.

56. Jafari S, Copes R, Baharlou S, Etminan M, Buxton J. Tattooing and the risk of transmission of hepatitis $\mathrm{C}$ : a systematic review and meta-analysis. Int J Infect Dis 2010;14:e928-e940.

57. Alter MJ. Epidemiology of hepatitis C. Hepatology 1997;26(3 Suppl 1):62S-65S.

58. Tomkins SE, Elford J, Nichols T, Aston J, Cliffe SJ, Roy K, et al. OC- cupational transmission of hepatitis $C$ in healthcare workers and factors associated with seroconversion: UK surveillance data. J Viral Hepat 2012;19:199-204.

59. Puro V, Petrosillo N, Ippolito G. Risk of hepatitis C seroconversion after occupational exposures in health care workers. Italian Study Group on Occupational Risk of HIV and Other Bloodborne Infections. Am J Infect Control 1995;23:273-277.

60. Lanphear BP, Linnemann CC Jr, Cannon CG, DeRonde MM, Pendy L, Kerley LM. Hepatitis C virus infection in healthcare workers: risk of exposure and infection. Infect Control Hosp Epidemiol 1994;15:745-750.

61. Ryoo SM, Kim WY, Kim W, Lim KS, Lee CC, Woo JH. Transmission of hepatitis $C$ virus by occupational percutaneous injuries in South Korea. J Formos Med Assoc 2012;111:113-117.

62. Tohme RA, Holmberg SD. Is sexual contact a major mode of hepatitis C virus transmission? Hepatology 2010;52:1497-1505.

63. Yaphe S, Bozinoff N, Kyle R, Shivkumar S, Pai NP, Klein M. Incidence of acute hepatitis $C$ virus infection among men who have sex with men with and without HIV infection: a systematic review. Sex Transm Infect 2012;88:558-564.

64. Prasad MR, Honegger JR. Hepatitis C virus in pregnancy. Am J Perinatol 2013;30:149-159.

65. Roberts EA, Yeung L. Maternal-infant transmission of hepatitis $C$ virus infection. Hepatology 2002;36(5 Suppl 1):S106-S113.

66. Indolfi $G$, Resti M. Perinatal transmission of hepatitis $C$ virus infection. J Med Virol 2009;81:836-843.

67. European Paediatric Hepatitis C Virus Network. A significant sex-but not elective cesarean section--effect on mother-to-child transmission of hepatitis C virus infection. J Infect Dis 2005;192:18721879.

68. Ghamar Chehreh ME, Tabatabaei SV, Khazanehdari S, Alavian SM. Effect of cesarean section on the risk of perinatal transmission of hepatitis C virus from HCV-RNA /HIV- mothers: a meta-analysis. Arch Gynecol Obstet 2011;283:255-260.

69. European Paediatric Hepatitis C Virus Network. Effects of mode of delivery and infant feeding on the risk of mother-to-child transmission of hepatitis C virus. European Paediatric Hepatitis C Virus Network. BJOG 2001;108:371-377.

70. Indolfi G, Nesi A, Resti M. Intrafamilial transmission of hepatitis $C$ virus. J Med Virol 2013;85:608-614.

71. Seong MH, Kil H, Kim YS, Bae SH, Lee YJ, Lee HC, et al. Clinical and epidemiological features of hepatitis $C$ virus infection in South Korea: a prospective, multicenter cohort study. J Med Virol 2013:85:1724-1733.

72. Farci P, Alter HJ, Wong D, Miller RH, Shih JW, Jett B, et al. A longterm study of hepatitis $C$ virus replication in non- $A$, non- $B$ hepatitis. N Engl J Med 1991;325:98-104.

73. Maasoumy B, Wedemeyer $\mathrm{H}$. Natural history of acute and chronic hepatitis C. Best Pract Res Clin Gastroenterol 2012;26:401-412. 
74. Corey KE, Mendez-Navarro J, Gorospe EC, Zheng H, Chung RT. Early treatment improves outcomes in acute hepatitis $C$ virus infection: a meta-analysis. J Viral Hepat 2010;17:201-207.

75. Santantonio T, Wiegand J, Gerlach JT. Acute hepatitis C: current status and remaining challenges. J Hepatol 2008;49:625-633.

76. Alter HJ, Seeff LB. Recovery, persistence, and sequelae in hepatitis $C$ virus infection: a perspective on long-term outcome. Semin Liver Dis 2000;20:17-35.

77. Gerlach JT, Diepolder HM, Zachoval R, Gruener NH, Jung MC, Ulsenheimer $A$, et al. Acute hepatitis $C$ : high rate of both spontaneous and treatment-induced viral clearance. Gastroenterology 2003;125:80-88.

78. Tremolada F, Casarin C, Alberti A, Drago C, Tagger A, Ribero ML, et al. Long-term follow-up of non-A, non-B (type $C$ ) post-transfusion hepatitis. J Hepatol 1992;16:273-281.

79. Lehmann M, Meyer MF, Monazahian M, Tillmann HL, Manns MP, Wedemeyer $\mathrm{H}$. High rate of spontaneous clearance of acute hepatitis C virus genotype 3 infection. J Med Virol 2004;73:387-391.

80. Hofer H, Watkins-Riedel T, Janata O, Penner E, Holzmann H, SteindlMunda $\mathrm{P}$, et al. Spontaneous viral clearance in patients with acute hepatitis $C$ can be predicted by repeated measurements of serum viral load. Hepatology 2003;37:60-64.

81. Kim KA, Lee JS, Yang JH, Moon YS, Lee WJ. Natural history of acute symptomatic hepatitis C in Korea. Korean J Gastroenterol 2005:46:105-109.

82. Kim JY, Won JE, Jeong SH, Park SJ, Hwang SG, Kang SK, et al. Acute hepatitis $\mathrm{C}$ in Korea: different modes of infection, high rate of spontaneous recovery, and low rate of seroconversion. J Med Virol 2011;83:1195-1202.

83. Thomas DL, Thio CL, Martin MP, Qi Y, Ge D, O'Huigin C, et al. Genetic variation in IL28B and spontaneous clearance of hepatitis $C$ virus. Nature 2009;461:798-801.

84. Tillmann HL, Thompson AJ, Patel K, Wiese M, Tenckhoff H, Nischalke HD, et al. A polymorphism near IL28B is associated with spontaneous clearance of acute hepatitis $C$ virus and jaundice. Gastroenterology 2010;139:1586-1592, 1592.e1.

85. Rao HY, Sun DG, Jiang D, Yang RF, Guo F, Wang JH, et al. IL28B genetic variants and gender are associated with spontaneous clearance of hepatitis C virus infection. J Viral Hepat 2012;19:173-181.

86. Tong MJ, el-Farra NS, Reikes AR, Co RL. Clinical outcomes after transfusion-associated hepatitis C. N Engl J Med 1995;332:14631466.

87. Thein HH, Yi Q, Dore GJ, Krahn MD. Estimation of stage-specific fibrosis progression rates in chronic hepatitis $C$ virus infection: a meta-analysis and meta-regression. Hepatology 2008:48:418-431.

88. Poynard T, Bedossa P, Opolon P. Natural history of liver fibrosis progression in patients with chronic hepatitis C. The OBSVIRC, METAVIR, CLINIVIR, and DOSVIRC groups. Lancet 1997;349:825-832.
89. Lok AS, Seeff LB, Morgan TR, di Bisceglie AM, Sterling RK, Curto $\mathrm{TM}$, et al. Incidence of hepatocellular carcinoma and associated risk factors in hepatitis C-related advanced liver disease. Gastroenterology 2009;136:138-148.

90. Sangiovanni A, Prati GM, Fasani P, Ronchi G, Romeo R, Manini M, et al. The natural history of compensated cirrhosis due to hepatitis C virus: A 17-year cohort study of 214 patients. Hepatology 2006:43:1303-1310.

91. Gomez EV, Rodriguez YS, Bertot LC, Gonzalez AT, Perez YM, Soler $E A$, et al. The natural history of compensated HCV-related cirrhosis: a prospective long-term study. J Hepatol 2013;58:434-444.

92. Fattovich G, Giustina G, Degos F, Tremolada F, Diodati G, Almasio $P$, et al. Morbidity and mortality in compensated cirrhosis type C: a retrospective follow-up study of 384 patients. Gastroenterology 1997;112:463-472.

93. Sinn DH, Paik SW, Kil JS, Kang P, Song SM, Gwak GY, et al. Incidence and risk factors for disease progression in Korean patients with chronic hepatitis C. Clin Mol Hepatol 2007;13(Suppl):S19.

94. Lee SS, Jeong SH, Jang ES, Kim YS, Lee YJ, Jung EU, et al. Prospective cohort study on the outcomes of hepatitis $C$ virus-related cirrhosis in South Korea. J Gastroenterol Hepatol 2015;30:1281-1287.

95. Zarski JP, Mc Hutchison J, Bronowicki JP, Sturm N, Garcia-Kennedy $R$, Hodaj $E$, et al. Rate of natural disease progression in patients with chronic hepatitis C. J Hepatol 2003;38:307-314.

96. Harris DR, Gonin R, Alter HJ, Wright EC, Buskell ZJ, Hollinger FB, et al. The relationship of acute transfusion-associated hepatitis to the development of cirrhosis in the presence of alcohol abuse. Ann Intern Med 2001;134:120-124.

97. Wiley TE, McCarthy M, Breidi L, McCarthy M, Layden TJ. Impact of alcohol on the histological and clinical progression of hepatitis $C$ infection. Hepatology 1998;28:805-809.

98. Noda K, Yoshihara H, Suzuki K, Yamada Y, Kasahara A, Hayashi N, et al. Progression of type $C$ chronic hepatitis to liver cirrhosis and hepatocellular carcinoma--its relationship to alcohol drinking and the age of transfusion. Alcohol Clin Exp Res 1996;20(1 Suppl):95A$100 \mathrm{~A}$.

99. Ortiz V, Berenguer M, Rayon JM, Carrasco D, Berenguer J. Contribution of obesity to hepatitis C-related fibrosis progression. Am J Gastroenterol 2002;97:2408-2414.

100. Ohki T, Tateishi R, Sato T, Masuzaki R, Imamura J, Goto T, et al. Obesity is an independent risk factor for hepatocellular carcinoma development in chronic hepatitis C patients. Clin Gastroenterol Hepatol 2008;6:459-464.

101. Adinolfi LE, Gambardella M, Andreana A, Tripodi MF, Utili R, Ruggiero $G$. Steatosis accelerates the progression of liver damage of chronic hepatitis C patients and correlates with specific HCV genotype and visceral obesity. Hepatology 2001;33:1358-1364.

102. Ohata K, Hamasaki K, Toriyama K, Matsumoto K, Saeki A, Yanagi 
The Korean Association for the Study of the Liver (KASL) KASL clinical practice guidelines: Management of Hepatitis C

$\mathrm{K}$, et al. Hepatic steatosis is a risk factor for hepatocellular carcinoma in patients with chronic hepatitis $C$ virus infection. Cancer 2003:97:3036-3043.

103. Poynard T, Ratziu V, Charlotte F, Goodman Z, McHutchison J, Albrecht J. Rates and risk factors of liver fibrosis progression in patients with chronic hepatitis c. J Hepatol 2001;34:730-739.

104. Imazeki F, Yokosuka O, Fukai K, Hiraide A, Saisho H. Significance of prior hepatitis $B$ virus infection in the development of hepatocellular carcinoma in patients with chronic hepatitis C. Dig Dis Sci 2003:48:1786-1792.

105. Tsai JF, Jeng JE, Ho MS, Chang WY, Lin ZY, Tsai JH. Independent and additive effect modification of hepatitis $C$ and $B$ viruses infection on the development of chronic hepatitis. J Hepatol 1996;24:271-276.

106. Vento S, Garofano T, Renzini C, Cainelli F, Casali F, Ghironzi G, et al. Fulminant hepatitis associated with hepatitis A virus superinfection in patients with chronic hepatitis C. N Engl J Med 1998;338:286-290.

107. Ghany MG, Strader DB, Thomas DL, Seeff LB. American Association for the Study of Liver Diseases. Diagnosis, management, and treatment of hepatitis C: an update. Hepatology 2009;49:13351374.

108. Smith BD, Morgan RL, Beckett GA, Falck-Ytter Y, Holtzman D, Ward JW. Hepatitis C virus testing of persons born during 19451965: recommendations from the Centers for Disease Control and Prevention. Ann Intern Med 2012;157:817-822.

109. Moyer VA. U.S. Preventive Services Task Force. Screening for hepatitis C virus infection in adults: U.S. Preventive Services Task Force recommendation statement. Ann Intern Med 2013;159:349-357.

110. Rein $D B$, Smith BD, Wittenborn JS, Lesesne $S B$, Wagner LD, Roblin $D W$, et al. The cost-effectiveness of birth-cohort screening for hepatitis C antibody in U.S. primary care settings. Ann Intern Med 2012;156:263-270.

111. Nakamura J, Terajima K, Aoyagi Y, Akazawa K. Cost-effectiveness of the national screening program for hepatitis $C$ virus in the general population and the high-risk groups. Tohoku J Exp Med 2008;215:33-42.

112. Sroczynski G, Esteban E, Conrads-Frank A, Schwarzer R, Muhlberger $N$, Wright $D$, et al. Long-term effectiveness and cost-effectiveness of screening for hepatitis $C$ virus infection. Eur J Public Health 2009;19:245-253.

113. Ki M, Choi HY, Jang ES, Jeong SH. Direct medical cost for hepatitis C virus infection in South Korea, 2009-2013 [Abstract]. The Liver Week 2015 2015:110A.

114. Kim DY, Han KH, Jun B, Kim TH, Park S, Ward T, et al. Cost-effectiveness of one-time screening for HCV in South Korea [Abstract]. The Liver Week 2015 2015:23A.

115. Pawlotsky JM. Molecular diagnosis of viral hepatitis. Gastroenter- ology 2002;122:1554- 1568.

116. Colin C, Lanoir D, Touzet S, Meyaud-Kraemer L, Bailly F, Trepo $C$, et al. Sensitivity and specificity of third-generation hepatitis $C$ virus antibody detection assays: an analysis of the literature. J Viral Hepat 2001;8:87-95.

117. Abdel-Hamid M, El-Daly M, El-Kafrawy S, Mikhail N, Strickland GT, Fix AD. Comparison of second- and third-generation enzyme immunoassays for detecting antibodies to hepatitis C virus. J Clin Microbiol 2002;40:1656-1659.

118. Pawlotsky JM. Use and interpretation of virological tests for hepatitis C. Hepatology 2002;36(5 Suppl 1):S65-S73.

119. Pawlotsky JM, Lonjon I, Hezode C, Raynard B, Darthuy F, Remire J, et al. What strategy should be used for diagnosis of hepatitis $C$ virus infection in clinical laboratories? Hepatology 1998;27:1700-1702.

120. Stramer SL, Caglioti S, Strong DM. NAT of the United States and Canadian blood supply. Transfusion 2000;40:1165-1168.

121. Alter MJ, Kuhnert WL, Finelli L. Centers for Disease Control and Prevention. Guidelines for laboratory testing and result reporting of antibody to hepatitis C virus. Centers for Disease Control and Prevention. MMWR Recomm Rep 2003;52(RR-3):1-13, 15;quiz CE11-14.

122. Moretti M, Pieretti B, Masucci A, Sisti D, Rocchi M, Delprete E. Role of signal-to-cutoff ratios in hepatitis $C$ virus antibody detection. Clin Vaccine Immunol 2012;19:1329-1331.

123. Lee SR, Kardos KW, Schiff E, Berne CA, Mounzer K, Banks AT, et al. Evaluation of a new, rapid test for detecting HCV infection, suitable for use with blood or oral fluid. J Virol Methods 2011;172:27-31.

124. European Association for Study of Liver. EASL Clinical Practice Guidelines: management of hepatitis C virus infection. J Hepatol 2014;60:392-420.

125. Alter MJ, Margolis HS, Krawczynski K, Judson FN, Mares A, Alexander WJ, et al. The natural history of community-acquired hepatitis $C$ in the United States. The Sentinel Counties Chronic non- $A$, non-B Hepatitis Study Team. N Engl J Med 1992;327:1899-1905.

126. Lauer GM, Walker BD. Hepatitis C virus infection. N Engl J Med 2001;345:41-52.

127. European Association for the Study of the Liver. EASL Clinical Practice Guidelines: management of hepatitis C virus infection. J Hepatol 2011;55:245-264.

128. Thio CL, Nolt KR, Astemborski J, Vlahov D, Nelson KE, Thomas DL. Screening for hepatitis $C$ virus in human immunodeficiency virusinfected individuals. J Clin Microbiol 2000;38:575- 577.

129. Meyer zum Buschenfelde KH, Gerken G, Manns M. Hepatitis $\mathrm{C}$ virus $(\mathrm{HCV})$ and autoimmune liver diseases. Arch Virol Suppl 1992;4:201-204.

130. Scott JD, Gretch DR. Molecular diagnostics of hepatitis C virus infection: a systematic review. JAMA 2007;297:724-732.

131. Sarrazin C, Teuber G, Kokka R, Rabenau H, Zeuzem S. Detection of residual hepatitis $C$ virus RNA by transcription-mediated ampli- 
fication in patients with complete virologic response according to polymerase chain reaction-based assays. Hepatology 2000;32(4 Pt 1):818- 823.

132. Martinot-Peignoux M, Boyer N, Le Breton V, Le Guludec G, Castelnau $C$, Akremi $R$, et al. A new step toward standardization of serum hepatitis $C$ virus-RNA quantification in patients with chronic hepatitis C. Hepatology 2000;31:726-729.

133. Pradat $P$, Chossegros P, Bailly F, Pontisso P, Saracco G, Sauleda S, et al. Comparison between three quantitative assays in patients with chronic hepatitis $C$ and their relevance in the prediction of response to therapy. J Viral Hepat 2000;7:203-210.

134. Chevaliez S, Bouvier-Alias M, Pawlotsky JM. Performance of the Abbott real-time PCR assay using m2000sp and m2000rt for hepatitis C virus RNA quantification. J Clin Microbiol 2009;47:17261732.

135. Vermehren J, Kau A, Gartner BC, Gobel R, Zeuzem S, Sarrazin C. Differences between two real-time PCR-based hepatitis $C$ virus (HCV) assays (RealTime HCV and Cobas AmpliPrep/ Cobas TaqMan) and one signal amplification assay (Versant HCV RNA 3.0) for RNA detection and quantification. J Clin Microbiol 2008:46:38803891.

136. Vermehren J, Yu ML, Monto A, Yao JD, Anderson C, Bertuzis R, et al. Multi-center evaluation of the Abbott RealTime HCV assay for monitoring patients undergoing antiviral therapy for chronic hepatitis C. J Clin Virol 2011;52:133-137.

137. Pawlotsky JM, Bouvier-Alias M, Hezode C, Darthuy F, Remire J, Dhumeaux D. Standardization of hepatitis C virus RNA quantification. Hepatology 2000;32:654-659.

138. Saldanha J, Lelie N, Heath A. Establishment of the first international standard for nucleic acid amplification technology (NAT) assays for HCV RNA. WHO Collaborative Study Group. Vox Sang 1999;76:149-158

139. Chevaliez S, Pawlotsky JM. Hepatitis C virus: virology, diagnosis and management of antiviral therapy. World J Gastroenterol 2007:13:2461-2466

140. Rehermann B, Nascimbeni M. Immunology of hepatitis B virus and hepatitis C virus infection. Nat Rev Immunol 2005;5:215-229.

141. Wieland SF, Chisari FV. Stealth and cunning: hepatitis B and hepatitis C viruses. J Virol 2005;79:9369-9380.

142. Nguyen TT, Sedghi-Vaziri A, Wilkes LB, Mondala T, Pockros PJ, Lindsay $\mathrm{KL}$, et al. Fluctuations in viral load (HCV RNA) are relatively insignificant in untreated patients with chronic HCV infection. J Viral Hepat 1996;3:75-78.

143. Ferreira-Gonzalez A, Shiffman ML. Use of diagnostic testing for managing hepatitis C virus infection. Semin Liver Dis 2004;24 Suppl 2:9-18.

144. Pawlotsky JM, Prescott L, Simmonds P, Pellet C, Laurent-Puig P, Labonne $C$, et al. Serological determination of hepatitis $C$ virus genotype: comparison with a standardized genotyping assay. J Clin Microbiol 1997;35:1734-1739.

145. Simmonds P, Bukh J, Combet C, Deleage G, Enomoto N, Feinstone S, et al. Consensus proposals for a unified system of nomenclature of hepatitis C virus genotypes. Hepatology 2005;42:962-973.

146. Chevaliez S, Pawlotsky JM. Diagnosis and management of chronic viral hepatitis: antigens, antibodies and viral genomes. Best Pract Res Clin Gastroenterol 2008;22:1031-1048.

147. Smith DB, Mellor J, Jarvis LM, Davidson F, Kolberg J, Urdea M, et al. Variation of the hepatitis $C$ virus 5 ' non-coding region: implications for secondary structure, virus detection and typing. The International HCV Collaborative Study Group. J Gen Virol 1995;76( Pt 7):1749- 1761.

148. Park JC, Kim JM, Kwon OJ, Lee KR, Chai YG, Oh HB. Development and clinical evaluation of a microarray for hepatitis $C$ virus genotyping. J Virol Methods 2010;163:269-275.

149. Chen Z, Weck KE. Hepatitis C virus genotyping: interrogation of the $5^{\prime}$ untranslated region cannot accurately distinguish genotypes 1a and 1b. J Clin Microbiol 2002;40:3127-3134.

150. Chevaliez S, Bouvier-Alias M, Brillet R, Pawlotsky JM. Hepatitis C virus (HCV) genotype 1 subtype identification in new HCV drug development and future clinical practice. PLoS One 2009;4:e8209.

151. Hong SK, Cho SI, Ra EK, Kim EC, Park JS, Park SS, et al. Evaluation of two hepatitis $C$ virus genotyping assays based on the $5^{\prime}$ untranslated region (UTR): the limitations of $5^{\prime}$ UTR-based assays and the need for a supplementary sequencing-based approach. J Clin Microbiol 2012;50:3741-3743.

152. Cai Q, Zhao Z, Liu Y, Shao X, Gao Z. Comparison of three different HCV genotyping methods: core, NS5B sequence analysis and line probe assay. Int J Mol Med 2013;31:347-352.

153. McCormick AL, Macartney MJ, Abdi-Abshir I, Labbett W, Smith C, Irish $D$, et al. Evaluation of sequencing of HCV core/E1, NS5A and NS5B as a genotype predictive tool in comparison with commercial assays targeting 5'UTR. J Clin Virol 2015;66:56-59.

154. Germer JJ, Rys PN, Thorvilson JN, Persing DH. Determination of hepatitis $C$ virus genotype by direct sequence analysis of products generated with the Amplicor HCV test. J Clin Microbiol 1999;37:2625-2630.

155. Romano KP, Ali A, Royer WE, Schiffer CA. Drug resistance against HCV NS3/4A inhibitors is defined by the balance of substrate recognition versus inhibitor binding. Proc Natl Acad Sci U S A 2010;107:20986-20991.

156. Kuntzen T, Timm J, Berical A, Lennon N, Berlin AM, Young SK, et al. Naturally occurring dominant resistance mutations to hepatitis $C$ virus protease and polymerase inhibitors in treatment-naive patients. Hepatology 2008;48:1769-1778.

157. Lenz O, Fevery B, Verbinnen T, Tambuyzer L, Vijgen L, Peeters M, et al. Resistance analyses of HCV isolates from patients treated 
The Korean Association for the Study of the Liver (KASL) KASL clinical practice guidelines: Management of Hepatitis C

with simeprevir in phase $2 \mathrm{~b} / 3$ studies. [Abstract]. Hepatology 2013;58:743A.

158. Lenz $O$, Verbinnen T, Fevery B, Tambuyzer L, Vijgen L, Peeters $M$, et al. Virology analyses of HCV isolates from genotype 1-infected patients treated with simeprevir plus PegIFN-a/ribavirin in Phase IIb/III studies. J Hepatol 2015;62:1008-1014.

159. Suzuki F, Sezaki H, Akuta N, Suzuki Y, Seko Y, Kawamura Y, et al. Prevalence of hepatitis C virus variants resistant to NS3 protease inhibitors or the NS5A inhibitor (BMS-790052) in hepatitis patients with genotype 1b. J Clin Virol 2012;54:352-354.

160. Karino Y, Toyota J, Ikeda K, Suzuki F, Chayama K, Kawakami Y, et al. Characterization of virologic escape in hepatitis $C$ virus genotype $1 \mathrm{~b}$ patients treated with the direct-acting antivirals daclatasvir and asunaprevir. J Hepatol 2013;58:646-654.

161. Pawlotsky JM. Drug resistance: prevalence and clinical implications during the treatment of chronic hepatitis C infection. Clin Liver Disease 2012;1:58-61.

162. Halfon P, Sarrazin C. Future treatment of chronic hepatitis $C$ with direct acting antivirals: is resistance important? Liver Int 2012;32 Suppl 1:79-87.

163. McPhee F, Suzuki Y, Toyota J, Karino Y, Chayama K, Kawakami $Y$, et al. High Sustained Virologic Response to Daclatasvir Plus Asunaprevir in Elderly and Cirrhotic Patients with Hepatitis C Virus Genotype 1b Without Baseline NS5A Polymorphisms. Adv Ther 2015;32:637-649.

164. McPhee F, Hernandez D, Yu F, Ueland J, Monikowski A, Carifa A, et al. Resistance analysis of hepatitis $C$ virus genotype 1 prior treatment null responders receiving daclatasvir and asunaprevir. Hepatology 2013;58:902-911.

165. Lok AS, Gardiner DF, Lawitz E, Martorell C, Everson GT, Ghalib R, et al. Preliminary study of two antiviral agents for hepatitis $C$ genotype 1. N Engl J Med 2012;366:216-224.

166. Chayama K, Takahashi S, Toyota J, Karino Y, Ikeda K, Ishikawa $\mathrm{H}$, et al. Dual therapy with the nonstructural protein $5 \mathrm{~A}$ inhibitor, daclatasvir, and the nonstructural protein 3 protease inhibitor, asunaprevir, in hepatitis C virus genotype $1 \mathrm{~b}$-infected null responders. Hepatology 2012;55:742-748.

167. Pasquinelli C, McPhee F, Eley T, Villegas C, Sandy K, Sheridan $P$, et al. Single- and multiple-ascending-dose studies of the NS3 protease inhibitor asunaprevir in subjects with or without chronic hepatitis C. Antimicrob Agents Chemother 2012;56:1838-1844.

168. Nguyen LT, Gray E, Dean J, Carr M, Connell J, De Gascun C, et al. Baseline prevalence and emergence of protease inhibitor resistance mutations following treatment in chronic HCV genotype 1-infected individuals. Antivir Ther 2015. [Epub ahead of print]

169. Shepherd SJ, Abdelrahman T, MacLean AR, Thomson EC, Aitken C, Gunson RN. Prevalence of HCV NS3 pre-treatment resistance associated amino acid variants within a Scottish cohort. J Clin Virol
2015;65:50-53.

170. Jensen SB, Serre SB, Humes DG, Ramirez S, Li YP, Bukh J, et al. Substitutions at Hepatitis C Virus Genotypes 2-6 NS3 Residues 155, 156, or 168 Induce Complex Patterns of Protease Inhibitor Resistance. Antimicrob Agents Chemother 2015;59:7426-7436.

171. Sarrazin C. The importance of resistance to direct antiviral drugs in HCV infection in clinical practice. J Hepatol 2015. [Epub ahead of print]

172. Lawitz EJ, Gruener D, Hill JM, Marbury T, Moorehead L, Mathias A, et al. A phase 1, randomized, placebo-controlled, 3-day, doseranging study of GS-5885, an NS5A inhibitor, in patients with genotype 1 hepatitis C. J Hepatol 2012;57:24-31.

173. Kwon HJ, Xing W, Chan K, Niedziela-Majka A, Brendza KM, Kirschberg $T$, et al. Direct binding of ledipasvir to HCV NS5A: mechanism of resistance to an HCV antiviral agent. PLoS One 2015;10:e0122844.

174. Alter MJ. The epidemiology of acute and chronic hepatitis C. Clin Liver Dis 1997;1:559-568, vi-vii.

175. U.S. Public Health Service. Updated U.S. Public Health Service Guidelines for the management of occupational exposures to HBV, $\mathrm{HCV}$, and HIV and recommendations for postexposure prophylaxis. MMWR Recomm Rep 2001;50(RR-11):1-52.

176. Smith BD, Morgan RL, Beckett GA, Falck-Ytter Y, Holtzman D, Teo CG, et al. Recommendations for the identification of chronic hepatitis C virus infection among persons born during 1945-1965. MMWR Recomm Rep 2012;61(RR-4):1-32.

177. Mitsui T, Iwano K, Masuko K, Yamazaki C, Okamoto H, Tsuda F, et al. Hepatitis $C$ virus infection in medical personnel after needlestick accident. Hepatology 1992;16:1109-1114.

178. Kubitschke A, Bahr MJ, Aslan N, Bader C, Tillmann HL, Sarrazin $C$, et al. Induction of hepatitis $C$ virus (HCV)-specific T cells by needle stick injury in the absence of HCV-viraemia. Eur J Clin Invest 2007;37:54-64.

179. Kleiner DE. The liver biopsy in chronic hepatitis C: a view from the other side of the microscope. Semin Liver Dis 2005;25:52-64.

180. Bedossa P, Poynard T. An algorithm for the grading of activity in chronic hepatitis C. The METAVIR Cooperative Study Group. Hepatology 1996;24:289-293.

181. Ishak K, Baptista A, Bianchi L, Callea F, De Groote J, Gudat F, et al. Histological grading and staging of chronic hepatitis. J Hepatol 1995;22:696-699.

182. Park YN, Kim Hg, Chon CY, Park JB, Sohn JH, Yang SH, et al. Histological grading and staging of chronic hepatitis standardized guideline proposed by the Korean study group for the pathology of digestive diseases. Korean J Pathol 1999;33:337-346.

183. Levine RA, Sanderson SO, Ploutz-Snyder R, Murray F, Kay E, Hegarty $\mathrm{J}$, et al. Assessment of fibrosis progression in untreated irish women with chronic hepatitis $C$ contracted from immunoglobulin 
anti-D. Clin Gastroenterol Hepatol 2006;4:1271-1277.

184. Thomas DL, Seeff LB. Natural history of hepatitis C. Clin Liver Dis 2005;9:383-398, vi.

185. Wong JB, Koff RS. Watchful waiting with periodic liver biopsy versus immediate empirical therapy for histologically mild chronic hepatitis C. A cost-effectiveness analysis. Ann Intern Med 2000;133:665-675.

186. Martinot-Peignoux M, Boyer N, Cazals-Hatem D, Pham BN, Gervais A, Le Breton $V$, et al. Prospective study on anti-hepatitis $C$ viruspositive patients with persistently normal serum alanine transaminase with or without detectable serum hepatitis C virus RNA. Hepatology 2001;34:1000-1005.

187. Shiffman ML, Diago M, Tran A, Pockros P, Reindollar R, Prati D, et al. Chronic hepatitis $C$ in patients with persistently normal alanine transaminase levels. Clin Gastroenterol Hepatol 2006;4:645-652.

188. Boccato S, Pistis R, Noventa F, Guido M, Benvegnu L, Alberti A. Fibrosis progression in initially mild chronic hepatitis C. J Viral Hepat 2006;13:297-302.

189. Yano M, Kumada H, Kage M, Ikeda K, Shimamatsu K, Inoue O, et al. The long-term pathological evolution of chronic hepatitis $C$. Hepatology 1996;23:1334-1340.

190. Fontaine $H$, Nalpas B, Poulet B, Carnot F, Zylberberg H, Brechot $C$, et al. Hepatitis activity index is a key factor in determining the natural history of chronic hepatitis C. Hum Pathol 2001;32:904909.

191. Rubbia-Brandt L, Fabris P, Paganin S, Leandro G, Male PJ, Giostra $E$, et al. Steatosis affects chronic hepatitis $C$ progression in a genotype specific way. Gut 2004;53:406-412.

192. Poynard T, Ratziu V, McHutchison J, Manns M, Goodman Z, Zeuzem $\mathrm{S}$, et al. Effect of treatment with PegIFN-a or interferon alfa$2 \mathrm{~b}$ and ribavirin on steatosis in patients infected with hepatitis $\mathrm{C}$. Hepatology 2003;38:75-85.

193. Olynyk JK, Reddy KR, Di Bisceglie AM, Jeffers LJ, Parker TI, Radick $J \mathrm{~L}$, et al. Hepatic iron concentration as a predictor of response to interferon alfa therapy in chronic hepatitis C. Gastroenterology 1995;108:1104-1109.

194. Westin J, Lagging M, Dhillon AP, Norkrans G, Romero Al, Pawlotsky JM, et al. Impact of hepatic steatosis on viral kinetics and treatment outcome during antiviral treatment of chronic HCV infection. J Viral Hepat 2007;14:29-35.

195. Patton HM, Patel K, Behling C, Bylund D, Blatt LM, Vallee M, et al. The impact of steatosis on disease progression and early and sustained treatment response in chronic hepatitis C patients. J Hepatol 2004;40:484-490.

196. Fontana RJ, Israel J, LeClair P, Banner BF, Tortorelli K, Grace N, et al. Iron reduction before and during interferon therapy of chronic hepatitis C: results of a multicenter, randomized, controlled trial. Hepatology 2000;31:730-736.
197. Reiss G, Keeffe EB. Role of liver biopsy in the management of chronic liver disease: selective rather than routine. Rev Gastroenterol Disord 2005;5:195-205.

198. Crockett SD, Kaltenbach T, Keeffe EB. Do we still need a liver biopsy? Are the serum fibrosis tests ready for prime time? Clin Liver Dis 2006;10:513-534, viii.

199. Dienstag JL. The role of liver biopsy in chronic hepatitis C. Hepatology 2002;36:S152-160.

200. Cadranel JF, Rufat P, Degos F. Practices of liver biopsy in France: results of a prospective nationwide survey. For the Group of Epidemiology of the French Association for the Study of the Liver (AFEF). Hepatology 2000;32:477-481.

201. Regev A, Berho M, Jeffers LJ, Milikowski C, Molina EG, Pyrsopoulos NT, et al. Sampling error and intraobserver variation in liver biopsy in patients with chronic HCV infection. Am J Gastroenterol 2002;97:2614-2618.

202. Rockey DC, Bissell DM. Noninvasive measures of liver fibrosis. Hepatology 2006;43(2 Suppl 1):S113-S120.

203. Poynard T, Ngo Y, Munteanu M, Thabut D, Massard J, Moussalli J, et al. Biomarkers of liver injury for hepatitis clinical trials: a metaanalysis of longitudinal studies. Antivir Ther 2010;15:617-631.

204. Colletta C, Smirne C, Fabris C, Toniutto P, Rapetti R, Minisini R, et al. Value of two noninvasive methods to detect progression of fibrosis among HCV carriers with normal aminotransferases. Hepatology 2005;42:838-845.

205. Halfon P, Bourliere M, Deydier R, Botta-Fridlund D, Renou C, Tran A, et al. Independent prospective multicenter validation of biochemical markers (fibrotest-actitest) for the prediction of liver fibrosis and activity in patients with chronic hepatitis C: the fibropaca study. Am J Gastroenterol 2006;101:547-555.

206. Parkes J, Guha IN, Roderick P, Harris S, Cross R, Manos MM, et al. Enhanced Liver Fibrosis (ELF) test accurately identifies liver fibrosis in patients with chronic hepatitis C. J Viral Hepat 2011;18:23-31.

207. Sheth SG, Flamm SL, Gordon FD, Chopra S. AST/ALT ratio predicts cirrhosis in patients with chronic hepatitis C virus infection. Am J Gastroenterol 1998;93:44-48.

208. Forns X, Ampurdanes S, Llovet JM, Aponte J, Quinto L, MartinezBauer $E$, et al. Identification of chronic hepatitis $C$ patients without hepatic fibrosis by a simple predictive model. Hepatology 2002;36:986-992.

209. Shaheen AA, Myers RP. Diagnostic accuracy of the aspartate aminotransferase-to-platelet ratio index for the prediction of hepatitis C-related fibrosis: a systematic review. Hepatology 2007;46:912921.

210. Poynard T, Imbert-Bismut F, Munteanu M, Messous D, Myers RP, Thabut $D$, et al. Overview of the diagnostic value of biochemical markers of liver fibrosis (FibroTest, HCV FibroSure) and necrosis (ActiTest) in patients with chronic hepatitis C. Comp Hepatol 
The Korean Association for the Study of the Liver (KASL) KASL clinical practice guidelines: Management of Hepatitis C

2004;3:8.

211. Adams LA, Bulsara M, Rossi E, DeBoer B, Speers D, George J, et al. Hepascore: an accurate validated predictor of liver fibrosis in chronic hepatitis C infection. Clin Chem 2005;51:1867-1873.

212. Boursier J, Bacq Y, Halfon P, Leroy V, de Ledinghen V, de Muret A, et al. Improved diagnostic accuracy of blood tests for severe fibrosis and cirrhosis in chronic hepatitis C. Eur J Gastroenterol Hepatol 2009;21:28-38.

213. Patel K, Benhamou Y, Yoshida EM, Kaita KD, Zeuzem S, Torbenson $M$, et al. An independent and prospective comparison of two commercial fibrosis marker panels (HCV FibroSURE and FIBROSpect II) during albinterferon alfa-2b combination therapy for chronic hepatitis C. J Viral Hepat 2009;16:178-186.

214. Wai CT, Greenson JK, Fontana RJ, Kalbfleisch JD, Marrero JA, Conjeevaram $\mathrm{HS}$, et al. A simple noninvasive index can predict both significant fibrosis and cirrhosis in patients with chronic hepatitis $C$. Hepatology 2003;38:518-526.

215. Park GJ, Lin BP, Ngu MC, Jones DB, Katelaris PH. Aspartate aminotransferase: alanine aminotransferase ratio in chronic hepatitis $C$ infection: is it a useful predictor of cirrhosis? J Gastroenterol Hepatol 2000;15:386-390.

216. Giannini E, Risso D, Botta F, Chiarbonello B, Fasoli A, Malfatti F, et al. Validity and clinical utility of the aspartate aminotransferasealanine aminotransferase ratio in assessing disease severity and prognosis in patients with hepatitis $C$ virus-related chronic liver disease. Arch Intern Med 2003;163:218-224.

217. Lin ZH, Xin YN, Dong QJ, Wang Q, Jiang XJ, Zhan SH, et al. Performance of the aspartate aminotransferase-to-platelet ratio index for the staging of hepatitis C-related fibrosis: an updated metaanalysis. Hepatology 2011;53:726-736.

218. Sterling RK, Lissen E, Clumeck N, Sola R, Correa MC, Montaner J, et al. Development of a simple noninvasive index to predict significant fibrosis in patients with HIV/HCV coinfection. Hepatology 2006;43:1317-1325.

219. Castera L, Vergniol J, Foucher J, Le Bail B, Chanteloup E, Haaser M, et al. Prospective comparison of transient elastography, Fibrotest, APRI, and liver biopsy for the assessment of fibrosis in chronic hepatitis C. Gastroenterology 2005;128:343-350.

220. Castera L. Transient elastography and other noninvasive tests to assess hepatic fibrosis in patients with viral hepatitis. J Viral Hepat 2009;16:300-314.

221. Jung KS, Kim SU. Clinical applications of transient elastography. Clin Mol Hepatol 2012;18:163-173.

222. Kim BK, Fung J, Yuen MF, Kim SU. Clinical application of liver stiffness measurement using transient elastography in chronic liver disease from longitudinal perspectives. World J Gastroenterol 2013;19:1890-1900.

223. Arena U, Vizzutti F, Corti G, Ambu S, Stasi C, Bresci S, et al. Acute viral hepatitis increases liver stiffness values measured by transient elastography. Hepatology 2008;47:380-384.

224. Sagir A, Erhardt A, Schmitt M, Haussinger D. Transient elastography is unreliable for detection of cirrhosis in patients with acute liver damage. Hepatology 2008;47:592-595.

225. Castera L, Forns X, Alberti A. Non-invasive evaluation of liver fibrosis using transient elastography. J Hepatol 2008;48:835-847.

226. Ziol M, Handra-Luca A, Kettaneh A, Christidis C, Mal F, Kazemi $F$, et al. Noninvasive assessment of liver fibrosis by measurement of stiffness in patients with chronic hepatitis C. Hepatology 2005;41:48-54.

227. Friedrich-Rust M, Ong MF, Martens S, Sarrazin C, Bojunga J, Zeuzem $S$, et al. Performance of transient elastography for the staging of liver fibrosis: a meta-analysis. Gastroenterology 2008;134:960974.

228. Degos F, Perez P, Roche B, Mahmoudi A, Asselineau J, Voitot $H$, et al. Diagnostic accuracy of FibroScan and comparison to liver fibrosis biomarkers in chronic viral hepatitis: a multicenter prospective study (the FIBROSTIC study). J Hepatol 2010;53:1013-1021.

229. Talwalkar JA, Yin M, Fidler JL, Sanderson SO, Kamath PS, Ehman RL. Magnetic resonance imaging of hepatic fibrosis: emerging clinical applications. Hepatology 2008;47:332-342.

230. Friedrich-Rust M, Nierhoff J, Lupsor M, Sporea I, FierbinteanuBraticevici C, Strobel D, et al. Performance of Acoustic Radiation Force Impulse imaging for the staging of liver fibrosis: a pooled meta-analysis. J Viral Hepat 2012;19:e212-e219.

231. Lee $Y$, Lee JM, Lee JE, Lee KB, Lee ES, Yoon JH, et al. MR elastography for noninvasive assessment of hepatic fibrosis: reproducibility of the examination and reproducibility and repeatability of the liver stiffness value measurement. J Magn Reson Imaging 2014;39:326331.

232. Swain MG, Lai MY, Shiffman ML, Cooksley WG, Zeuzem S, Dieterich DT, et al. A sustained virologic response is durable in patients with chronic hepatitis $C$ treated with PegIFN-a alfa-2a and ribavirin. Gastroenterology 2010;139:1593-1601.

233. Manns M, Pol S, Jacobson IM, Marcellin P, Gordon SC, Peng CY, et al. All-oral daclatasvir plus asunaprevir for hepatitis $C$ virus genotype 1b: a multinational, phase 3, multicohort study. Lancet 2014;384:1597-1605.

234. Sulkowski MS, Gardiner DF, Rodriguez-Torres M, Reddy KR, Hassanein $T$, Jacobson I, et al. Daclatasvir plus sofosbuvir for previously treated or untreated chronic HCV infection. N Engl J Med 2014;370:211-221.

235. Shiratori Y, Imazeki F, Moriyama M, Yano M, Arakawa Y, Yokosuka 0 , et al. Histologic improvement of fibrosis in patients with hepatitis $C$ who have sustained response to interferon therapy. Ann Intern Med 2000;132:517-524.

236. Poynard T, McHutchison J, Manns M, Trepo C, Lindsay K, Goodman 
$Z$, et al. Impact of pegylated interferon alfa-2b and ribavirin on liver fibrosis in patients with chronic hepatitis C. Gastroenterology 2002;122:1303-1313.

237. Pradat P, Tillmann HL, Sauleda S, Braconier JH, Saracco G, Thursz $\mathrm{M}$, et al. Long-term follow-up of the hepatitis C HENCORE cohort: response to therapy and occurrence of liver-related complications. J Viral Hepat 2007;14:556-563.

238. Shiratori Y, Ito Y, Yokosuka O, Imazeki F, Nakata R, Tanaka N, et al. Antiviral therapy for cirrhotic hepatitis $C$ : association with reduced hepatocellular carcinoma development and improved survival. Ann Intern Med 2005;142:105-114.

239. Ogawa E, Furusyo N, Kajiwara E, Takahashi K, Nomura H, Maruyama $T$, et al. Efficacy of pegylated interferon alpha-2b and ribavirin treatment on the risk of hepatocellular carcinoma in patients with chronic hepatitis C: a prospective, multicenter study. J Hepatol 2013;58:495501.

240. Deuffic-Burban S, Deltenre P, Louvet A, Canva V, Dharancy S, Hollebecque $A$, et al. Impact of viral eradication on mortality related to hepatitis C: a modeling approach in France. J Hepatol 2008;49:175183.

241. Bruno S, Stroffolini T, Colombo M, Bollani S, Benvegnu L, Mazzella $G$, et al. Sustained virological response to interferon-alpha is associated with improved outcome in HCV-related cirrhosis: a retrospective study. Hepatology 2007;45:579-587.

242. Fabrizi F, Dixit V, Messa P. Antiviral therapy of symptomatic HCVassociated mixed cryoglobulinemia: meta-analysis of clinical studies. J Med Virol 2013;85:1019-1027.

243. Feng B, Eknoyan G, Guo ZS, Jadoul M, Rao HY, Zhang W, et al. Effect of interferon-alpha- based antiviral therapy on hepatitis $C$ virus-associated glomerulonephritis: a meta-analysis. Nephrol Dial Transplant 2012;27:640-646.

244. Everson GT, Trotter J, Forman L, Kugelmas M, Halprin A, Fey B, et al. Treatment of advanced hepatitis $C$ with a low accelerating dosage regimen of antiviral therapy. Hepatology 2005;42:255-262.

245. Berenguer M, Palau A, Aguilera V, Rayón JM, Juan FS, Prieto $M$. Clinical benefits of antiviral therapy in patients with recurrent hepatitis C following liver transplantation. Am J Transplant 2008;8:679-687.

246. Hagihara H, Nouso K, Kobayashi Y, Iwasaki Y, Nakamura S, Kuwaki $\mathrm{K}$, et al. Effect of pegylated interferon therapy on intrahepatic recurrence after curative treatment of hepatitis $C$ virus-related hepatocellular carcinoma. Int J Clin Oncol 2011;16:210-220.

247. Singal AK, Freeman DH Jr, Anand BS. Meta-analysis: interferon improves outcomes following ablation or resection of hepatocellular carcinoma. Aliment Pharmacol Ther 2010;32:851-858.

248. Chen J, Florian J, Carter W, Fleischer RD, Hammerstrom TS, Jadhav $P R$, et al. Earlier sustained virologic response end points for regulatory approval and dose selection of hepatitis $C$ therapies. Gastro- enterology 2013;144:1450-1455.e2.

249. Mangia A, Minerva N, Bacca D, Cozzolongo R, Ricci GL, Carretta $V$, et al. Individualized treatment duration for hepatitis $C$ genotype 1 patients: A randomized controlled trial. Hepatology 2008;47:4350.

250. Jensen DM, Morgan TR, Marcellin P, Pockros PJ, Reddy KR, Hadziyannis SJ, et al. Early identification of HCV genotype 1 patients responding to 24 weeks PegIFN-a-2a (40 kd)/ribavirin therapy. Hepatology 2006;43:954-960.

251. Yu JW, Wang GQ, Sun LJ, Li XG, Li SC. Predictive value of rapid virological response and early virological response on sustained virological response in HCV patients treated with pegylated interferon alpha-2a and ribavirin. J Gastroenterol Hepatol 2007;22:832-836.

252. Yu ML, Dai CY, Huang JF, Chiu CF, Yang YH, Hou NJ, et al. Rapid virological response and treatment duration for chronic hepatitis C genotype 1 patients: a randomized trial. Hepatology 2008;47:1884-1893.

253. Andriulli A, Mangia A, lacobellis A, Ippolito A, Leandro G, Zeuzem S. Meta-analysis: the outcome of anti-viral therapy in HCV genotype 2 and genotype 3 infected patients with chronic hepatitis. Aliment Pharmacol Ther 2008;28:397-404.

254. Fried MW, Shiffman ML, Reddy KR, Smith C, Marinos G, Goncales $\mathrm{FL} \mathrm{Jr}$, et al. Peginterferon alfa-2a plus ribavirin for chronic hepatitis C virus infection. N Engl J Med 2002;347:975- 982.

255. Davis GL, Wong JB, McHutchison JG, Manns MP, Harvey J, Albrecht J. Early virologic response to treatment with PegIFN-a alfa$2 \mathrm{~b}$ plus ribavirin in patients with chronic hepatitis C. Hepatology 2003;38:645-652.

256. Ferenci P, Fried MW, Shiffman ML, Smith Cl, Marinos G, Goncales $\mathrm{FL} \mathrm{Jr}$, et al. Predicting sustained virological responses in chronic hepatitis C patients treated with PegIFN-a alfa-2a (40 KD)/ribavirin. J Hepatol 2005;43:425-433.

257. Berg T, von Wagner M, Nasser S, Sarrazin C, Heintges T, Gerlach $T$, et al. Extended treatment duration for hepatitis $C$ virus type 1: comparing 48 versus 72 weeks of PegIFN-a-alfa-2a plus ribavirin. Gastroenterology 2006;130:1086-1097.

258. Pearlman BL, Ehleben C, Saifee S. Treatment extension to 72 weeks of PeglFN-a and ribavirin in hepatitis c genotype 1-infected slow responders. Hepatology 2007:46:1688-1694.

259. Lawitz E, Mangia A, Wyles D, Rodriguez-Torres M, Hassanein $T$, Gordon SC, et al. Sofosbuvir for previously untreated chronic hepatitis C infection. N Engl J Med 2013;368:1878-1887.

260. Forns $X$, Lawitz E, Zeuzem S, Gane E, Bronowicki JP, Andreone $P$, et al. Simeprevir with PegIFN-a and ribavirin leads to high rates of SVR in patients with HCV genotype 1 who relapsed after previous therapy: a phase 3 trial. Gastroenterology 2014;146:1669-1679.e3.

261. Manns MP, McHutchison JG, Gordon SC, Rustgi VK, Shiffman M, Reindollar $\mathrm{R}$, et al. Peginterferon alfa-2b plus ribavirin compared 
The Korean Association for the Study of the Liver (KASL) KASL clinical practice guidelines: Management of Hepatitis C with interferon alfa-2b plus ribavirin for initial treatment of chronic hepatitis C: a randomised trial. Lancet 2001;358:958-965.

262. Hadziyannis SJ, Sette H Jr, Morgan TR, Balan V, Diago M, Marcellin $P$, et al. Peginterferon-alpha2a and ribavirin combination therapy in chronic hepatitis C: a randomized study of treatment duration and ribavirin dose. Ann Intern Med 2004;140:346-355.

263. Thompson AJ, Muir AJ, Sulkowski MS, Ge D, Fellay J, Shianna $\mathrm{KV}$, et al. Interleukin-28B polymorphism improves viral kinetics and is the strongest pretreatment predictor of sustained virologic response in genotype 1 hepatitis C virus. Gastroenterology 2010;139:120-129.e18.

264. Ge D, Fellay J, Thompson AJ, Simon JS, Shianna KV, Urban TJ, et al. Genetic variation in IL28B predicts hepatitis C treatment-induced viral clearance. Nature 2009:461:399-401.

265. Park SH, Park CK, Lee JW, Kim YS, Jeong SH, Kim YS, et al. Efficacy and tolerability of PegIFN-a plus ribavirin in the routine daily treatment of chronic hepatitis C patients in Korea: a multi-center, retrospective observational study. Gut Liver 2012;6:98-106.

266. von Wagner M, Huber M, Berg T, Hinrichsen H, Rasenack J, Heintges $T$, et al. Peginterferon- alpha-2a (40KD) and ribavirin for 16 or 24 weeks in patients with genotype 2 or 3 chronic hepatitis C. Gastroenterology 2005;129:522-527.

267. Muir AJ, Bornstein JD, Killenberg PG. Atlantic Coast Hepatitis Treatment Group. Peginterferon alfa-2b and ribavirin for the treatment of chronic hepatitis $\mathrm{C}$ in blacks and non-Hispanic whites. N Engl J Med 2004;350:2265-2271.

268. Dai CY, Huang JF, Hsieh MY, Hou NJ, Lin ZY, Chen SC, et al. Insulin resistance predicts response to PegIFN-a-alpha/ribavirin combination therapy in chronic hepatitis C patients. J Hepatol 2009;50:712718.

269. Romero-Gomez M, Del Mar Viloria M, Andrade RJ, Salmeron J, Diago M, Fernandez- Rodriguez CM, et al. Insulin resistance impairs sustained response rate to PegIFN-a plus ribavirin in chronic hepatitis C patients. Gastroenterology 2005;128:636-641.

270. Tanaka Y, Nishida N, Sugiyama M, Kurosaki M, Matsuura K, Sakamoto N, et al. Genome- wide association of IL28B with response to pegylated interferon-alpha and ribavirin therapy for chronic hepatitis C. Nat Genet 2009;41:1105-1109.

271. Mangia A, Thompson AJ, Santoro R, Piazzolla V, Tillmann HL, Patel $\mathrm{K}$, et al. An IL28B polymorphism determines treatment response of hepatitis C virus genotype 2 or 3 patients who do not achieve a rapid virologic response. Gastroenterology 2010;139:821-827, 827. e1.

272. Lyoo K, Song MJ, Hur W, Choi JE, Hong SW, Kim CW, et al. Polymorphism near the IL28B gene in Korean hepatitis C virus-infected patients treated with peg-interferon plus ribavirin. J Clin Virol 2011;52:363-366

273. Jeong SH, Jung YK, Yang JW, Park SJ, Kim JW, Kwon OS, et al.
Efficacy of PegIFN-a and ribavirin is associated with the IL28B gene in Korean patients with chronic hepatitis C. Clin Mol Hepatol 2012;18:360-367.

274. Jung YK, Kim JH, Ahn SM, Yang JW, Park SJ, Kim JW, et al. Role of interleukin 28B-related gene polymorphisms in chronic hepatitis $C$ and the response to antiviral therapy in Koreans. J Clin Gastroenterol 2013;47:644-650.

275. McHutchison JG, Manns M, Patel K, Poynard T, Lindsay KL, Trepo $C$, et al. Adherence to combination therapy enhances sustained response in genotype-1-infected patients with chronic hepatitis $C$. Gastroenterology 2002;123:1061-1069.

276. Afdhal N, Zeuzem S, Kwo P, Chojkier M, Gitlin N, Puoti M, et al. Ledipasvir and sofosbuvir for untreated HCV genotype 1 infection. N Engl J Med 2014;370:1889-1898.

277. Alqahtani SA, Afdhal N, Zeuzem S, Gordon SC, Mangia A, Kwo P, et al. Safety and tolerability of ledipasvir/sofosbuvir with and without ribavirin in patients with chronic hepatitis C virus genotype 1 infection: Analysis of phase III ION trials. Hepatology 2015;62:2530.

278. Jacobson IM, Kwo PY, Kowdley KV, Yang JC, Zhu Y, Hyland RH, et al. Virologic response rates to all oral fixed-dose combination ledipasvir/sofosbuvir regimens are similar in patients with and without traditional negative predictive factors in phase 3 clinical trials [Abstract]. Hepatology 2014;60:1141A-1142A.

279. Bourliere M, Sulkowski MS, Omata M, Zeuzem S, Feld JJ, Lawitz E, et al. An integrated safety and efficacy analysis of $\square 500$ patients with compensated cirrhosis treated with ledipasvir/sofosbuvir with or without ribavirin [Abstract]. Hepatology 2014;60:239A.

280. Feld JJ, Kowdley KV, Coakley E, Sigal S, Nelson DR, Crawford D, et al. Treatment of HCV with ABT-450/r-ombitasvir and dasabuvir with ribavirin. N Engl J Med 2014;370:1594-1603.

281. Ferenci P, Bernstein D, Lalezari J, Cohen D, Luo Y, Cooper C, et al. ABT-450/r-ombitasvir and dasabuvir with or without ribavirin for HCV. N Engl J Med 2014;370:1983-1992.

282. Poordad F, Hezode C, Trinh R, Kowdley KV, Zeuzem S, Agarwal K, et al. ABT-450/r-ombitasvir and dasabuvir with ribavirin for hepatitis C with cirrhosis. N Engl J Med 2014;370:1973-1982.

283. Feld J, Moreno C, Trinh R, Tam E, Bourgeois S, Horsmans Y, et al. Turquoise-III: safety and efficacy of 12-week ribavirin-free treatment for patients with HCV genotype $1 \mathrm{~b}$ and cirrhosis [Abstract]. J Viral Hepat 2015;22:135A.

284. Kao JH, Peng CY, Chang TT, Heo J, Chu CJ, Lee YJ, et al. All-oral dual Therapy with daclatasvir and asunaprevir in patients in Korea and Taiwan with HCV genotype 1b infection [Abstract]. Hepatol Int 2015:9:74A-75A.

285. Lawitz E, Sulkowski MS, Ghalib R, Rodriguez-Torres M, Younossi ZM, Corregidor A, et al. Simeprevir plus sofosbuvir, with or without ribavirin, to treat chronic infection with hepatitis $C$ virus genotype 
1 in non-responders to pegylated interferon and ribavirin and treatment-naive patients: the COSMOS randomised study. Lancet 2014;384:1756-1765.

286. Kwo P, Gitlin N, Nahass R, Bernstein D, Rojter S, Schiff E, et al. A Phase 3, randomised, open-label study to evaluate the efficacy and safety of 12 and 8 weeks of simeprevir (SMV) plus sofosbuvir (SOF) in treatment-naïve and-experienced patients with chronic HCV genotype 1 infection without cirrhosis: OPTIMIST-1 [Abstract]. J Hepatol 2015;62:270A.

287. Lawitz E, Matusow G, De Jesus E, Yoshida E, Felizarta F, Ghalib R, et al. A phase 3, open-label, single-arm study to evaluate the efficacy and safety of 12 weeks of simeprevir (SMV) plus sofosbuvir (SOF) in treatment-naïve or-experienced patients with chronic HCV genotype 1 infection and cirrhosis: Optimist-2 [Abstract]. J Hepatol 2015;62:264A-265A.

288. Dieterich D, Bacon BR, Flamm SL, Kowdley KV, Milligan S, Tsai N, et al. Evaluation of sofosbuvir and simeprevir-based regimens in the TRIO network: academic and community treatment of a real-world, heterogeneous population [Abstract]. Hepatology 2014;60:220A.

289. Kowdley KV, Lawitz E, Crespo I, Hassanein T, Davis MN, DeMicco $M$, et al. Sofosbuvir with pegylated interferon alfa-2a and ribavirin for treatment-naive patients with hepatitis $C$ genotype-1 infection (ATOMIC): an open-label, randomised, multicentre phase 2 trial. Lancet 2013;381:2100-2107.

290. Jacobson IM, Dore GJ, Foster GR, Fried MW, Radu M, Rafalsky VV, et al. Simeprevir with pegylated interferon alfa 2a plus ribavirin in treatment-naive patients with chronic hepatitis C virus genotype 1 infection (QUEST-1): a phase 3, randomised, double-blind, placebocontrolled trial. Lancet 2014;384:403-413.

291. Manns M, Marcellin P, Poordad F, de Araujo ES, Buti M, Horsmans $Y$, et al. Simeprevir with pegylated interferon alfa $2 \mathrm{a}$ or $2 \mathrm{~b}$ plus ribavirin in treatment-naive patients with chronic hepatitis $C$ virus genotype 1 infection (QUEST-2): a randomised, double-blind, placebo- controlled phase 3 trial. Lancet 2014;384:414-426.

292. Afdhal NH, McHutchison JG, Zeuzem S, Mangia A, Pawlotsky JM, Murray JS, et al. Hepatitis C pharmacogenetics: state of the art in 2010. Hepatology 2011;53:336-345.

293. Jeong SW, Kim JD, Woo HY, You CR, Lee SW, Song MJ, et al. Impact of adherence to PegIFN-a-ribavirin combination therapy in chronic hepatitis $C$ patients on achieving a sustained virologic response. Korean J Hepatol 2009;15:338-349.

294. Kang MJ, Jung EU, Park SW, Choi P, Kim JH, Park SJ, et al. Effects of pegylated interferon and ribavirin in Korean patients with chronic hepatitis C virus infection. Korean J Hepatol 2008;14:318-330.

295. Kwon JH, Bae SH, Choi JY, Yoon SK, Byun KS, Paik SW, et al. Assessment of the efficacy of reducing PegIFN-a-2a and ribavirin dose on virologic response in Koreans with chronic hepatitis C. Korean J Intern Med 2009;24:203-211.
296. Park SH, Park CK, Lee JW, Kim YS, Jeong SH, Kim YS, et al. Efficacy and tolerability of PegIFN-a plus ribavirin in the routine daily treatment of chronic hepatitis C patients in Korea: a multi-center, retrospective observational study. Gut Liver 2012;6:98-106.

297. Sinn DH, Shin SR, Kil JS, Kim J, Gwak GY, Choi MS, et al. Efficacy of peg-interferon-alpha-2a plus ribavirin for patients aged 60 years and older with chronic hepatitis C in Korea. J Gastroenterol Hepatol 2011;26:469-476.

298. Park SY, Rim MY, Yo IK, Ha MS, Kim JS, Lee JW, et al. Efficacy of PegIFN-a and ribavirin combination therapy of chronic hepatitis C: a pooled analysis. Korean J Gastroenterol 2012;60:306-314.

299. Berg T, Sarrazin C, Herrmann E, Hinrichsen H, Gerlach T, Zachoval $\mathrm{R}$, et al. Prediction of treatment outcome in patients with chronic hepatitis C: significance of baseline parameters and viral dynamics during therapy. Hepatology 2003;37:600-609.

300. Afdhal N, Reddy KR, Nelson DR, Lawitz E, Gordon SC, Schiff E, et al. Ledipasvir and sofosbuvir for previously treated HCV genotype 1 infection. N Engl J Med 2014;370:1483-1493.

301. Ahn SH, Jeong S, Paik S, Yang J, Mo H, Ga B, et al. Korean patients with genotype 1 and 2 HCV infection achieved over $97 \%$ sustained virologic response following 12 weeks of ledipasvir/sofosbuvir or sofosbuvir plus ribavirin [Abstract]. Hepatol Int 2015;9:S72.

302. Mizokami M, Yokosuka O, Takehara T, Sakamoto N, Korenaga M, Mochizuki $\mathrm{H}$, et al. Ledipasvir and sofosbuvir fixed-dose combination with and without ribavirin for 12 weeks in treatment-naive and previously treated Japanese patients with genotype 1 hepatitis C: an open-label, randomised, phase 3 trial. Lancet Infect Dis 2015;15:645-653.

303. Bourlière $M$, Bronowicki JP, de Ledinghen V, Hézode C, Zoulim F, Mathurin $\mathrm{P}$, et al. Ledipasvir-sofosbuvir with or without ribavirin to treat patients with HCV genotype 1 infection and cirrhosis nonresponsive to previous protease-inhibitor therapy: a randomised, double-blind, phase 2 trial (SIRIUS). Lancet Infect Dis 2015;15:397404.

304. Zeuzem S, Jacobson IM, Baykal T, Marinho RT, Poordad F, Bourlière $M$, et al. Retreatment of HCV with ABT-450/r-ombitasvir and dasabuvir with ribavirin. N Engl J Med 2014;370:1604-1614.

305. Andreone P, Colombo MG, Enejosa JV, Koksal I, Ferenci P, Maieron A, et al. ABT-450, ritonavir, ombitasvir, and dasabuvir achieves $97 \%$ and $100 \%$ sustained virologic response with or without ribavirin in treatment-experienced patients with HCV genotype $1 \mathrm{~b}$ infection. Gastroenterology 2014;147:359-365.e1.

306. Kumada H, Suzuki Y, Ikeda K, Toyota J, Karino Y, Chayama K, et al. Daclatasvir plus asunaprevir for chronic HCV genotype $1 \mathrm{~b}$ infection. Hepatology 2014;59:2083-2091.

307. Pol S, Bourliere M, Lucier S, De Ledinghen V, Zoulim F, DorivalMouly $C$, et al. Safety and efficacy of the combination daclatasvirsofosbuvir in HCV genotype 1-mono-infected patients from the 
The Korean Association for the Study of the Liver (KASL) KASL clinical practice guidelines: Management of Hepatitis C

French observational cohort ANRS CO22 HEPATHER [Abstract]. J Hepatol 2015;62 (Suppl 2):S258.

308. Reddy KR, Zeuzem S, Zoulim F, Weiland O, Horban A, Stanciu C, et al. Simeprevir versus telaprevir with PegIFN-a and ribavirin in previous null or partial responders with chronic hepatitis $C$ virus genotype 1 infection (ATTAIN): a randomised, double-blind, noninferiority phase 3 trial. Lancet Infect Dis 2015;15:27-35.

309. Kao JH, Ahn SH, Chien RN, Jeong SH, Peng CY, Lim YS, et al. P0809: 98\% SVR12 in Korean and Taiwanese patients with chronic genotype 2 HCV infection receiving 12 weeks of sofosbuvir plus ribavirin: results from an international, multicenter phase 3 study. J Hepatol 2015;62:S638.

310. Jacobson IM, Gordon SC, Kowdley KV, Yoshida EM, RodriguezTorres M, Sulkowski MS, et al. Sofosbuvir for hepatitis $C$ genotype 2 or 3 in patients without treatment options. N Engl J Med 2013;368:1867-1877.

311. Wyles DL, Ruane PJ, Sulkowski MS, Dieterich D, Luetkemeyer A, Morgan TR, et al. Daclatasvir plus Sofosbuvir for HCV in patients coinfected with HIV-1. N Engl J Med 2015;373:714-725.

312. Jacobson IM, Brown RS Jr, Freilich B, Afdhal N, Kwo PY, Santoro J, et al. Peginterferon alfa-2b and weight-based or flat-dose ribavirin in chronic hepatitis C patients: a randomized trial. Hepatology 2007;46:971-981

313. Zeuzem S, Hultcrantz R, Bourliere M, Goeser T, Marcellin P, Sanchez-Tapias J, et al. Peginterferon alfa-2b plus ribavirin for treatment of chronic hepatitis $C$ in previously untreated patients infected with HCV genotypes 2 or 3. J Hepatol 2004;40:993-999.

314. Ferenci P, Brunner H, Laferl H, Scherzer TM, Maieron A, Strasser M, et al. A randomized, prospective trial of ribavirin $400 \mathrm{mg} /$ day versus $800 \mathrm{mg} /$ day in combination with PeglFN-a alfa-2a in hepatitis C virus genotypes 2 and 3. Hepatology 2008;47:1816-1823.

315. Lee S, Kim IH, Kim SH, Kim SW, Lee SO, Lee ST, et al. Efficacy and tolerability of pegylated interferon-alpha2a plus ribavirin versus pegylated interferon-alpha2b plus ribavirin in treatment-naive chronic hepatitis C patients. Intervirology 2010;53:146-153.

316. Diago M, Shiffman ML, Bronowicki JP, Zeuzem S, Rodriguez-Torres M, Pappas SC, et al. Identifying hepatitis C virus genotype $2 / 3$ patients who can receive a 16-week abbreviated course of PegIFN-a alfa-2a (40KD) plus ribavirin. Hepatology 2010;51:1897-1903.

317. Lagging $M$, Langeland $N$, Pedersen C, Färkkilä M, Buw MR, Mørch $\mathrm{K}$, et al. Randomized comparison of 12 or 24 weeks of PegIFN-a$2 a$ and ribavirin in chronic hepatitis C virus genotype 2/3 infection. Hepatology 2008:47:1837-1845.

318. Poynard T, Munteanu M, Colombo M, Bruix J, Schiff E, Terg R, et al. FibroTest is an independent predictor of virologic response in chronic hepatitis $C$ patients retreated with pegylated interferon al$\mathrm{fa}-2 \mathrm{~b}$ and ribavirin in the EPIC(3) program. J Hepatol 2011;54:227235.
319. Mangia A, Santoro R, Minerva N, Ricci GL, Carretta V, Persico M, et al. Peginterferon alfa-2b and ribavirin for 12 vs. 24 weeks in HCV genotype 2 or 3. N Engl J Med 2005;352:2609-2617.

320. Shiffman ML, Suter F, Bacon BR, Nelson D, Harley $H$, Solá $R$, et al. Peginterferon alfa-2a and ribavirin for 16 or 24 weeks in HCV genotype 2 or 3. N Engl J Med 2007;357:124-134.

321. Aghemo A, Rumi MG, Monico S, Prati GM, D'Ambrosio R, Donato $M F$, et al. The pattern of pegylated interferon-alpha2 $b$ and ribavirin treatment failure in cirrhotic patients depends on hepatitis $C$ virus genotype. Antivir Ther 2009;14:577-584.

322. Marciano S, Gadano AC. How to optimize current treatment of genotype 2 hepatitis C virus infection. Liver Int 2014;34 Suppl 1:1317.

323. Marcellin $P$, Cheinquer $H$, Curescu M, Dusheiko GM, Ferenci $P$, Horban A, et al. High sustained virologic response rates in rapid virologic response patients in the large real-world PROPHESYS cohort confirm results from randomized clinical trials. Hepatology 2012;56:2039-2050.

324. Yu ML, Dai CY, Huang JF, Hou NJ, Lee LP, Hsieh MY, et al. A randomised study of PegIFN-a and ribavirin for 16 versus 24 weeks in patients with genotype 2 chronic hepatitis C. Gut 2007;56:553559.

325. Dalgard O, Bjøro K, Ring-Larsen H, Bjornsson E, Holberg-Petersen $M$, Skovlund $E$, et al. Pegylated interferon alfa and ribavirin for 14 versus 24 weeks in patients with hepatitis C virus genotype 2 or 3 and rapid virological response. Hepatology 2008;47:35-42.

326. Mecenate F, Barbaro G, Pellicelli A, Barlattani A, Mazzoni E, Bonaventura $M E$, et al. Comparison of peg-interferon alfa-2a and ribavirin for 12 or 24 weeks in patients with HCV genotype 2 or 3 : The cleo trial. Hepatology 2007:46:828A.

327. Zeuzem S, Dusheiko GM, Salupere R, Mangia A, Flisiak R, Hyland $\mathrm{RH}$, et al. Sofosbuvir and ribavirin in HCV genotypes 2 and 3. N Engl J Med 2014;370:1993-2001.

328. Omata M, Nishiguchi S, Ueno Y, Mochizuki H, Izumi N, Ikeda F, et al. $97 \%$ Sustained virologic response in Japanese patients with chronic genotype 2 hepatitis C virus infection receiving sofosbuvir in combination with ribavirin for 12 weeks: results from a phase 3 multicenter study. Hepatology 2014;60:671A.

329. Shiffman ML, Di Bisceglie AM, Lindsay KL, Morishima C, Wright EC, Everson GT, et al. Peginterferon alfa-2a and ribavirin in patients with chronic hepatitis $C$ who have failed prior treatment. Gastroenterology 2004;126:1015-1023; discussion 947.

330. Jacobson IM, Gonzalez SA, Ahmed F, Lebovics E, Min AD, Bodenheimer $\mathrm{HC} J$ r, et al. A randomized trial of pegylated interferon alpha-2b plus ribavirin in the retreatment of chronic hepatitis $C$. Am J Gastroenterol 2005;100:2453-2462.

331. Taliani G, Gemignani G, Ferrari C, Aceti A, Bartolozzi D, Blanc PL, et al. Pegylated interferon alfa-2b plus ribavirin in the retreatment 
of interferon-ribavirin nonresponder patients. Gastroenterology 2006;130:1098-1106.

332. Sherman M, Yoshida EM, Deschenes M, Krajden M, Bain VG, Peltekian $\mathrm{K}$, et al. Peginterferon alfa-2a (40KD) plus ribavirin in chronic hepatitis $C$ patients who failed previous interferon therapy. Gut 2006;55:1631-1638.

333. Gonçales FL Jr, Moma CA, Vigani AG, Angerami AF, Gonçales ES, Tozzo $R$, et al. Retreatment of hepatitis $C$ patients with pegylated interferon combined with ribavirin in non-responders to interferon plus ribavirin. Is it different in real life? BMC Infect Dis 2010;10:212.

334. Sagir A, Heintges T, Akyazi Z, Oette M, Erhardt A, Häussinger D. Relapse to prior therapy is the most important factor for the retreatment response in patients with chronic hepatitis $C$ virus infection. Liver Int 2007;27:954-959.

335. Krawitt EL, Ashikaga T, Gordon SR, Ferrentino N, Ray MA, Lidofsky $S D$, et al. Peginterferon alfa-2b and ribavirin for treatment-refractory chronic hepatitis C. J Hepatol 2005;43:243-249.

336. Cheruvattath R, Rosati MJ, Gautam M, Vargas HE, Rakela J, Balan V. Pegylated interferon and ribavirin failures: is retreatment an option? Dig Dis Sci 2007;52:732-736.

337. Foster GR, Pianko S, Cooper C, Brown A, Forton D, Nahass RG. Sofosbuvir plus Peg-IFN/ribavirin for 12 weeks vs sofosbuvir/ribavirin for 16 or 24 weeks in genotype 3 HCV-infected patients and treatment-experienced cirrhotic patients with genotype $2 \mathrm{HCV}$ : the Boson Study. J Hepatol 2015;62:S259-S260.

338. Nelson DR, Cooper JN, Lalezari JP, Lawitz E, Pockros PJ, Gitlin N, et al. All-oral 12-week treatment with daclatasvir plus sofosbuvir in patients with hepatitis C virus genotype 3 infection: ALLY-3 phase III study. Hepatology 2015;61:1127-1135.

339. Hezode C, De Ledinghen V, Fontaine $H$, Zoulim F, Lebray P, Boyer $N$, et al. LP05: Daclatasvir plus sofosbuvir with or without ribavirin in patients with HCV genotype 3 infection: interim analysis of a French multicenter compassionate use program. J Hepatol 2015;62:S265-S266

340. Gane EJ, Hyland RH, An D, Svarovskaia E, Pang PS, Brainard D, et al. Efficacy of ledipasvir and sofosbuvir, with or without ribavirin, for 12 weeks in patients with HCV genotype 3 or 6 infection. Gastroenterology 2015;149:1454-1461,e1.

341. Lawitz E, Lalezari JP, Hassanein T, Kowdley KV, Poordad FF, Sheikh AM, et al. Sofosbuvir in combination with PegIFN-a alfa-2a and ribavirin for non-cirrhotic, treatment-naive patients with genotypes 1, 2, and 3 hepatitis C infection: a randomised, double-blind, phase 2 trial. Lancet Infect Dis 2013:13:401-408.

342. Gane EJ, Stedman CA, Hyland RH, Ding X, Svarovskaia E, Symonds WT, et al. Nucleotide polymerase inhibitor sofosbuvir plus ribavirin for hepatitis C. N Engl J Med 2013;368:34-44.

343. Lawitz E, Poordad F, Brainard DM, Hyland RH, An D, Dvory-Sobol H, et al. Sofosbuvir with PegIFN-a-ribavirin for 12 weeks in previously treated patients with hepatitis C genotype 2 or 3 and cirrhosis. Hepatology 2015;61:769-775.

344. Poynard T, Colombo M, Bruix J, Schiff E, Terg R, Flamm S, et al. Peginterferon alfa-2b and ribavirin: effective in patients with hepatitis $C$ who failed interferon alfa/ribavirin therapy. Gastroenterology 2009;136:1618-1628. e2.

345. Alfaleh FZ, Hadad Q, Khuroo MS, Aljumah A, Algamedi A, Alashgar $\mathrm{H}$, et al. Peginterferon alpha-2b plus ribavirin compared with interferon alpha-2b plus ribavirin for initial treatment of chronic hepatitis C in Saudi patients commonly infected with genotype 4. Liver Int 2004;24:568-574.

346. Ruane PJ, Ain D, Stryker R, Meshrekey R, Soliman M, Wolfe PR, et al. Sofosbuvir plus ribavirin for the treatment of chronic genotype 4 hepatitis C virus infection in patients of Egyptian ancestry. J Hepatol 2015;62:1040-1046.

347. Doss W, Shiha G, Hassany M, Soliman R, Fouad R, Khairy M, et al. Sofosbuvir plus ribavirin for treating Egyptian patients with hepatitis C genotype 4. J Hepatol 2015;63:581-585.

348. Molina JM, Orkin C, Iser DM, Zamora FX, Nelson M, Stephan C, et al. Sofosbuvir plus ribavirin for treatment of hepatitis $C$ virus in patients co-infected with HIV (PHOTON-2): a multicentre, open-label, non-randomised, phase 3 study. Lancet 2015;385:1098-1106.

349. Kohli A, Kapoor R, Sims Z, Nelson A, Sidharthan S, Lam B, et al. Ledipasvir and sofosbuvir for hepatitis $C$ genotype 4: a proof-ofconcept, single-centre, open-label phase 2a cohort study. Lancet Infect Dis 2015;15:1049-1054.

350. Hézode C, Asselah T, Reddy KR, Hassanein T, Berenguer M, Fleischer-Stepniewska K, et al. Ombitasvir plus paritaprevir plus ritonavir with or without ribavirin in treatment-naive and treatment-experienced patients with genotype 4 chronic hepatitis C virus infection (PEARL-I): a randomised, open-label trial. Lancet 2015;385:25022509.

351. Moreno C, Hezode C, Marcellin P, Bourgeois S, Francque S, Samuel $D$, et al. Efficacy and safety of simeprevir with PegIFN/ribavirin in naïve or experienced patients infected with chronic HCV genotype 4. J Hepatol 2015;62:1047-1055.

352. Nguyen MH, Keeffe EB. Chronic hepatitis C: genotypes 4 to 9. Clin Liver Dis 2005;9:411-426, vi.

353. Yuen MF, Lai CL. Response to combined interferon and ribavirin is better in patients infected with hepatitis C virus genotype 6 than genotype 1 in Hong Kong. Intervirology 2006;49:96-98.

354. Tsang OT, Zee JS, Chan JM, Li RS, Kan YM, Li FT, et al. Chronic hepatitis $C$ genotype 6 responds better to pegylated interferon and ribavirin combination therapy than genotype 1. J Gastroenterol Hepatol 2010;25:766-771.

355. Nguyen NH, VuTien P, Garcia RT, Trinh H, Nguyen H, Nguyen K, et al. Response to pegylated interferon and ribavirin in Asian American patients with chronic hepatitis C genotypes 1 vs $2 / 3$ vs 6 . J 
The Korean Association for the Study of the Liver (KASL) KASL clinical practice guidelines: Management of Hepatitis C
Viral Hepat 2010;17:691-697.

356. Charlton M, Everson GT, Flamm SL, Kumar P, Landis C, Brown RS Jr, et al. Ledipasvir and sofosbuvir plus ribavirin for treatment of HCV infection in patients with advanced liver disease. Gastroenterology 2015;149:649-659.

357. Poordad F, Schiff ER, Vierling JM, Landis C, Fontana RJ, Yang R, et al. L08: Daclatasvir, sofosbuvir, and ribavirin combination for HCV patients with advanced cirrhosis or posttransplant recurrence: phase 3 ALLY-1 study. J Hepatol 2015;62: Suppl 261-S262.

358. Saxena V, Nyberg L, Pauly M, Dasgupta A, Nyberg A, Piasecki B, et al. Safety and efficacy of simeprevir/sofosbuvir in hepatitis Cinfected patients with compensated and decompensated cirrhosis. Hepatology 2015;62:715-725.

359. Cheong HR, Woo HY, Heo J, Yoon KT, Kim DU, Kim GH, et al. Clinical efficacy and safety of the combination therapy of PegIFN-a and ribavirin in cirrhotic patients with HCV infection. Korean J Hepatol 2010;16:38-48

360. Garcia-Retortillo M, Forns X, Feliu A, Moitinho E, Costa J, Navasa $M$, et al. Hepatitis $C$ virus kinetics during and immediately after liver transplantation. Hepatology 2002;35:680-687.

361. Forman LM, Lewis JD, Berlin JA, Feldman HI, Lucey MR. The association between hepatitis $C$ infection and survival after orthotopic liver transplantation. Gastroenterology 2002;122:889-896.

362. Prieto M, Berenguer M, Rayón JM, Córdoba J, Argüello L, Carrasco $D$, et al. High incidence of allograft cirrhosis in hepatitis $C$ virus genotype $1 \mathrm{~b}$ infection following transplantation: relationship with rejection episodes. Hepatology 1999;29:250-256.

363. Gane E, Pilmore H. Management of chronic viral hepatitis before and after renal transplantation. Transplantation 2002;74:427-437.

364. Curry MP, Forns X, Chung RT, Terrault NA, Brown R Jr, Fenkel JM, et al. Sofosbuvir and ribavirin prevent recurrence of HCV infection after liver transplantation: an open-label study. Gastroenterology 2015;148:100-107.e1.

365. Pungpapong S, Aqel B, Leise M, Werner KT, Murphy JL, Henry TM, et al. Multicenter experience using simeprevir and sofosbuvir with or without ribavirin to treat hepatitis C genotype 1 after liver transplant. Hepatology 2015;61:1880-1886.

366. Kwo PY, Mantry PS, Coakley E, Te HS, Vargas HE, Brown R Jr, et al. An interferon-free antiviral regimen for $\mathrm{HCV}$ after liver transplantation. N Engl J Med 2014;371:2375-2382.

367. Samuel D, Bizollon T, Feray C, Roche B, Ahmed SN, Lemonnier C, et al. Interferon-alpha $2 \mathrm{~b}$ plus ribavirin in patients with chronic hepatitis $C$ after liver transplantation: a randomized study. Gastroenterology 2003;124:642-650.

368. Carrión JA, Navasa M, García-Retortillo M, García-Pagan JC, Crespo $G$, Bruguera $M$, et al. Efficacy of antiviral therapy on hepatitis $C$ recurrence after liver transplantation: a randomized controlled study. Gastroenterology 2007;132:1746-1756.
369. Angelico M, Petrolati A, Lionetti R, Lenci I, Burra P, Donato MF, et al. A randomized study on Peg-interferon alfa-2a with or without ribavirin in liver transplant recipients with recurrent hepatitis C. J Hepatol 2007;46:1009-1017.

370. Martin P, Fabrizi F. Hepatitis C virus and kidney disease. J Hepatol 2008;49:613-624.

371. Bonacci M, Londoño MC, Esforzado N, Forns X, Sotoca JM, Campistol JM. Antiviral treatment with sofosbuvir and simeprevir in a kidney transplant recipient with HCV-decompensated cirrhosis: viral eradication and removal from the liver transplant waiting list. Transpl Int 2015;28:1345-1349.

372. Huard G, Kim B, Patel A, Aljarallah B, Perumalswami P, Odin JA, et al. Early safety and efficacy profiles of renal transplant recipients with chronic hepatitis C treated with Sofosbuvir and Ribavirin. Hepatology 2014;60(S1):540A-541A.

373. Licata A, Di Bona D, Schepis F, Shahied L, Craxí A, Cammà C. When and how to treat acute hepatitis C? J Hepatol 2003;39:10561062.

374. Wang CC, Krantz E, Klarquist J, Krows M, McBride L, Scott EP, et al. Acute hepatitis $\mathrm{c}$ in a contemporary US cohort: modes of acquisition and factors influencing viral clearance. J Infect Dis 2007;196:1474-1482.

375. McGovern BH, Nagami EH, Birch CE, Bowen MJ, Reyor LL, Chung $R T$, et al. Rate of sustained virologic response in relation to baseline hepatitis C virus (HCV) RNA level and rapid virologic clearance in persons with acute HCV infection. J Infect Dis 2009;200:877881.

376. Kamal SM, Fouly AE, Kamel RR, Hockenjos B, Al Tawil A, Khalifa $K E$, et al. Peginterferon alfa-2b therapy in acute hepatitis $C$ : impact of onset of therapy on sustained virologic response. Gastroenterology 2006;130:632-638.

377. Deuffic-Burban S, Castel H, Wiegand J, Manns MP, Wedemeyer H, Mathurin $\mathrm{P}$, et al. Immediate vs. delayed treatment in patients with acute hepatitis C based on IL28B polymorphism: a model-based analysis. J Hepatol 2012;57:260-266.

378. Deterding K, Grüner N, Buggisch P, Wiegand J, Galle PR, Spengler U, et al. Delayed versus immediate treatment for patients with acute hepatitis C: a randomised controlled non-inferiority trial. Lancet Infect Dis 2013;13:497-506.

379. Jaeckel E, Cornberg M, Wedemeyer H, Santantonio T, Mayer J, Zankel $\mathrm{M}$, et al. Treatment of acute hepatitis $\mathrm{C}$ with interferon alfa2b. N Engl J Med 2001;345:1452-1457.

380. Nomura H, Sou S, Tanimoto H, Nagahama T, Kimura Y, Hayashi J, et al. Short-term interferon-alfa therapy for acute hepatitis C: a randomized controlled trial. Hepatology 2004;39:1213-1219.

381. Kamal SM, Ismail A, Graham CS, He Q, Rasenack JW, Peters T, et al. Pegylated interferon alpha therapy in acute hepatitis C: relation to hepatitis C virus-specific T cell response kinetics. Hepatology 
2004;39:1721-1731.

382. Broers B, Helbling B, François A, Schmid P, Chuard C, Hadengue A, et al. Barriers to interferon-alpha therapy are higher in intravenous drug users than in other patients with acute hepatitis C. J Hepatol 2005;42:323-328.

383. Santantonio T, Fasano M, Sinisi E, Guastadisegni A, Casalino C, Mazzola $M$, et al. Efficacy of a 24-week course of PEG-interferon alpha-2b monotherapy in patients with acute hepatitis $C$ after failure of spontaneous clearance. J Hepatol 2005;42:329-333.

384. Wiegand J, Buggisch P, Boecher W, Zeuzem S, Gelbmann CM, Berg $T$, et al. Early monotherapy with pegylated interferon alpha-2b for acute hepatitis C infection: the HEP-NET acute-HCV-II study. Hepatology 2006;43:250-256.

385. Kamal SM, Moustafa KN, Chen J, Fehr J, Abdel Moneim A, Khalifa $K E$, et al. Duration of PegIFN-a therapy in acute hepatitis $\mathrm{C}$ : a randomized trial. Hepatology 2006;43:923-931.

386. Doyle JS, Deterding K, Grebely J, Wedemeyer H, Sacks-Davis R, Spelman $\mathrm{T}$, et al. Response to treatment following recently acquired hepatitis $C$ virus infection in a multicentre collaborative cohort. J Viral Hepat 2015;22:1020-1032.

387. Santantonio T, Fasano M, Sagnelli E, Tundo P, Babudieri S, Fabris $P$, et al. Acute hepatitis C: a 24-week course of pegylated interferon $\alpha-2 b$ versus a 12-week course of pegylated interferon $\alpha-2 b$ alone or with ribavirin. Hepatology 2014;59:2101-2109.

388. Sublette VA, Douglas MW, McCaffery K, George J, Perry KN. Psychological, lifestyle and social predictors of hepatitis $C$ treatment response: a systematic review. Liver Int 2013;33: 894-903.

389. Mishra P, Florian J, Qi K, Zeng W, Naeger LK, Donaldson E, et al. FDA perspective on sofosbuvir therapy for patients with chronic hepatitis $C$ virus genotype 1 infection who did not respond to treatment with pegylated interferon and ribavirin. Gastroenterology 2014;147:1196-1200.

390. Pol S, Sulkowski MS, Hassanein T, Gane EJ, Liu L, Mo H, et al. Sofosbuvir plus pegylated interferon and ribavirin in patients with genotype 1 hepatitis C virus in whom previous therapy with directacting antivirals has failed. Hepatology 2015;62:129-134.

391. Steinebrunner N, Sprinzl MF, Zimmermann T, Wörns MA, Zimmerer $T$, Galle PR, et al. Early virological response may predict treatment response in sofosbuvir-based combination therapy of chronic hepatitis c in a multi-center "real-life" cohort. BMC Gastroenterol 2015;15:97.

392. AASLD/IDSA HCV Guidance Panel. Hepatitis C guidance: AASLDIDSA recommendations for testing, managing, and treating adults infected with hepatitis C virus. Hepatology 2015;62:932-954.

393. Backus LI, Boothroyd DB, Phillips BR, Belperio P, Halloran J, Mole LA. A sustained virologic response reduces risk of all-cause mortality in patients with hepatitis C. Clin Gastroenterol Hepatol 2011;9:509-516.e1.
394. Backmund M, Meyer K, Edlin BR. Infrequent reinfection after successful treatment for hepatitis $C$ virus infection in injection drug users. Clin Infect Dis 2004;39:1540-1543.

395. Dalgard O. Follow-up studies of treatment for hepatitis C virus infection among injection drug users. Clin Infect Dis 2005;40 Suppl 5:S336-S338

396. Currie SL, Ryan JC, Tracy D, Wright TL, George S, McQuaid R, et al. A prospective study to examine persistent HCV reinfection in injection drug users who have previously cleared the virus. Drug Alcohol Depend 2008;93:148-154.

397. Grebely J, Knight E, Ngai T, Genoway KA, Raffa JD, Storms M, et al. Reinfection with hepatitis $C$ virus following sustained virological response in injection drug users. J Gastroenterol Hepatol 2010;25:1281-1284.

398. Manns MP, Wedemeyer H, Cornberg M. Treating viral hepatitis C: efficacy, side effects, and complications. Gut 2006;55:1350-1359.

399. Thévenot T, Cadranel JF, Di Martino V, Pariente A, Causse X, Renou $C$, et al. A national French survey on the use of growth factors as adjuvant treatment of chronic hepatitis C. Hepatology 2007;45:377383.

400. McHutchison JG, Dusheiko G, Shiffman ML, Rodriguez-Torres M, Sigal S, Bourliere $M$, et al. Eltrombopag for thrombocytopenia in patients with cirrhosis associated with hepatitis C. N Engl J Med 2007:357:2227-2236.

401. Afdhal NH, Giannini EG, Tayyab G, Mohsin A, Lee JW, Andriulli A, et al. Eltrombopag before procedures in patients with cirrhosis and thrombocytopenia. N Engl J Med 2012;367:716-724.

402. Janssen HL, Brouwer JT, van der Mast RC, Schalm SW. Suicide associated with alfa-interferon therapy for chronic viral hepatitis. J Hepatol 1994;21:241-243.

403. Udina M, Castellví $P$, Moreno-España J, Navinés $R$, Valdés $M$, Forns $X$, et al. Interferon-induced depression in chronic hepatitis C: a systematic review and meta-analysis. J Clin Psychiatry 2012;73:11281138.

404. Musselman DL, Lawson DH, Gumnick JF, Manatunga AK, Penna $\mathrm{S}$, Goodkin RS, et al. Paroxetine for the prevention of depression induced by high-dose interferon alfa. N Engl J Med 2001;344:961966.

405. de Knegt RJ, Bezemer G, Van Gool AR, Drenth JP, Hansen BE, Droogleever Fortuyn $\mathrm{HA}$, et al. Randomised clinical trial: escitalopram for the prevention of psychiatric adverse events during treatment with PegIFN-a-alfa-2a and ribavirin for chronic hepatitis C. Aliment Pharmacol Ther 2011;34:1306-1317.

406. Tomer Y, Blackard JT, Akeno N. Interferon alpha treatment and thyroid dysfunction. Endocrinol Metab Clin North Am 2007;36:10511066; $x-x i$

407. Mandac JC, Chaudhry S, Sherman KE, Tomer Y. The clinical and physiological spectrum of interferon-alpha induced thyroiditis: to- 
The Korean Association for the Study of the Liver (KASL) KASL clinical practice guidelines: Management of Hepatitis C

ward a new classification. Hepatology 2006;43:661-672.

408. Marazuela M, García-Buey L, González-Fernández B, GarcíaMonzón C, Arranz A, Borque MJ, et al. Thyroid autoimmune disorders in patients with chronic hepatitis $C$ before and during interferon-alpha therapy. Clin Endocrinol (Oxf) 1996;44:635-642.

409. Lisker-Melman M, Di Bisceglie AM, Usala SJ, Weintraub B, Murray LM, Hoofnagle JH. Development of thyroid disease during therapy of chronic viral hepatitis with interferon alfa. Gastroenterology 1992;102:2155-2160.

410. Martocchia A, Labbadia G, Paoletti V, Gargano S, Grossi A, Trabace $S$, et al. Hashimoto's disease during interferon-alpha therapy in a patient with pre-treatment negative anti-thyroid autoantibodies and with the specific genetic susceptibility to the thyroid disease. Neuro Endocrinol Lett 2001;22:49-52.

411. Carella C, Mazziotti G, Morisco F, Manganella G, Rotondi M, Tuccillo $C$, et al. Long-term outcome of interferon-alpha-induced thyroid autoimmunity and prognostic influence of thyroid autoantibody pattern at the end of treatment. J Clin Endocrinol Metab 2001;86:1925-1929.

412. Wong V, Fu AX, George J, Cheung NW. Thyrotoxicosis induced by alpha-interferon therapy in chronic viral hepatitis. Clin Endocrinol (Oxf) 2002;56:793-798.

413. Gutkowski K, Gutkowska D, Bilkiewicz T. Interferon therapy in chronic viral hepatitis; an autoimmunity dilemma. Przegl Lek 2007;64:148-152.

414. Panetta JD, Gilani N. Interferon-induced retinopathy and its risk in patients with diabetes and hypertension undergoing treatment for chronic hepatitis C virus infection. Aliment Pharmacol Ther 2009;30:597-602.

415. Malik NN, Sheth HG, Ackerman N, Davies N, Mitchell SM. A prospective study of change in visual function in patients treated with pegylated interferon alpha for hepatitis $\mathrm{C}$ in the UK. Br J Ophthalmol 2008;92:256-258.

416. Chisholm JA, Williams G, Spence E, Parks S, Keating D, Gavin M, et al. Retinal toxicity during pegylated alpha-interferon therapy for chronic hepatitis C: a multifocal electroretinogram investigation. Aliment Pharmacol Ther 2005;21:723-732.

417. Sene D, Touitou V, Bodaghi B, Saadoun D, Perlemuter G, Cassoux N, et al. Intraocular complications of IFN-alpha and ribavirin therapy in patients with chronic viral hepatitis C. World J Gastroenterol 2007;13:3137-3140.

418. Vujosevic S, Tempesta D, Noventa F, Midena E, Sebastiani G. Pegylated interferon-associated retinopathy is frequent in hepatitis $\mathrm{C}$ virus patients with hypertension and justifies ophthalmologic screening. Hepatology 2012;56:455-463.

419. Okuse C, Yotsuyanagi H, Nagase Y, Kobayashi Y, Yasuda K, Koike $K$, et al. Risk factors for retinopathy associated with interferon alpha-2b and ribavirin combination therapy in patients with chronic hepatitis C. World J Gastroenterol 2006;12:3756-3759.

420. Schulman JA, Liang C, Kooragayala LM, King J. Posterior segment complications in patients with hepatitis $C$ treated with interferon and ribavirin. Ophthalmology 2003;110:437-442.

421. Formann E, Stauber R, Denk DM, Jessner W, Zollner G, MundaSteindl $P$, et al. Sudden hearing loss in patients with chronic hepatitis $C$ treated with pegylated interferon/ribavirin. Am J Gastroenterol 2004;99:873-877.

422. De Franceschi L, Fattovich G, Turrini F, Ayi K, Brugnara C, Manzato $F$, et al. Hemolytic anemia induced by ribavirin therapy in patients with chronic hepatitis C virus infection: role of membrane oxidative damage. Hepatology 2000;31:997-1004.

423. Afdhal NH, Dieterich DT, Pockros PJ, Schiff ER, Shiffman ML, Sulkowski MS, et al. Epoetin alfa maintains ribavirin dose in HCVinfected patients: a prospective, double-blind, randomized controlled study. Gastroenterology 2004;126:1302-1311.

424. Kochhar DM, Penner JD, Knudsen TB. Embryotoxic, teratogenic, and metabolic effects of ribavirin in mice. Toxicol Appl Pharmacol 1980;52:99-112.

425. Bravo MJ, Vallejo F, Barrio G, Brugal MT, Molist G, Pulido J, et al. $\mathrm{HCV}$ seroconversion among never-injecting heroin users at baseline: no predictors identified other than starting injection. Int J Drug Policy 2012;23:415-419.

426. Armstrong GL, Wasley A, Simard EP, McQuillan GM, Kuhnert WL, Alter MJ. The prevalence of hepatitis C virus infection in the United States, 1999 through 2002. Ann Intern Med 2006;144:705-714.

427. Kim HS, Choo DH. Prevalence of anti-HCV among drug users in Korea. Korean J Med 1996;50:194-200.

428. Dimova RB, Zeremski M, Jacobson IM, Hagan H, Des Jarlais DC, Talal AH. Determinants of hepatitis $C$ virus treatment completion and efficacy in drug users assessed by meta-analysis. Clin Infect Dis 2013;56:806-816.

429. European Association for Study of Liver. EASL Recommendations on Treatment of Hepatitis C 2015. J Hepatol 2015;63:199-236.

430. Grebely J, Robaeys G, Bruggmann P, Aghemo A, Backmund M, Bruneau J, et al. Recommendations for the management of hepatitis C virus infection among people who inject drugs. Int J Drug Policy 2015;26:1028-1038.

431. Garimella T, Wang R, Luo WL, Wastall P, Kandoussi H, Demicco M, et al. Evaluation of drug-drug interaction between daclatasvir and methadone or buprenorphine/naloxone. J Int AIDS Soc 2014;17(4 Suppl 3):19628.

432. Garimella T, Wang R, Luo WL, Wastall P, Kandoussi H, DeMicco M, et al. Assessment of drug-drug interactions between daclatasvir and methadone or buprenorphine-naloxone. Antimicrob Agents Chemother 2015;59:5503-5510.

433. Menon RM, Badri PS, Wang T, Polepally AR, Zha J, Khatri A, et al. Drug-drug interaction profile of the all-oral anti-hepatitis $C$ 
virus regimen of paritaprevir/ritonavir, ombitasvir, and dasabuvir. J Hepatol 2015;63:20-29.

434. Lalezari J, Sullivan JG, Varunok P, Galen E, Kowdley KV, Rustgi V, et al. Ombitasvir/paritaprevir/r 4 and dasabuvir plus ribavirin in HCV genotype 1-infected patients on methadone or buprenorphine. J Hepatol 2015;63:364-369.

435. Liu CH, Kao JH. Treatment of hepatitis C virus infection in patients with end-stage renal disease. J Gastroenterol Hepatol 2011;26:228239.

436. Bang BK, Choi BS, Kim HW, Kim SK, Yang CW, Kim YS, et al. Retrospective study on the impact of hepatitis $B$ and hepatitis $C$ virus infection on renal transplnat recipients over 15 years. Korean J Nephrol 2002;21:423-434.

437. Kidney Disease: Improving Global Outcomes (KDIGO). KDIGO clinical practice guidelines for the prevention, diagnosis, evaluation, and treatment of hepatitis C in chronic kidney disease. Kidney Int Suppl 2008;(109):S1-99.

438. Marcelli D, Stannard D, Conte F, Held PJ, Locatelli F, Port FK. ESRD patient mortality with adjustment for comorbid conditions in Lombardy (Italy) versus the United States. Kidney Int 1996;50:10131018.

439. Maisonneuve P, Agodoa L, Gellert R, Stewart JH, Buccianti $G$, Lowenfels $A B$, et al. Cancer in patients on dialysis for endstage renal disease: an international collaborative study. Lancet 1999;354:93-99.

440. Nakayama E, Akiba T, Marumo F, Sato C. Prognosis of antihepatitis $C$ virus antibody-positive patients on regular hemodialysis therapy. J Am Soc Nephrol 2000;11:1896-1902.

441. Bruchfeld A, Wilczek H, Elinder CG. Hepatitis C infection, time in renal-replacement therapy, and outcome after kidney transplantation. Transplantation 2004;78:745-750.

442. Aroldi A, Lampertico P, Montagnino G, Passerini P, Villa M, Campise MR, et al. Natural history of hepatitis $B$ and $C$ in renal allograft recipients. Transplantation 2005;79:1132-1136.

443. Bloom RD, Rao V, Weng F, Grossman RA, Cohen D, Mange KC. Association of hepatitis $C$ with posttransplant diabetes in renal transplant patients on tacrolimus. J Am Soc Nephrol 2002;13:13741380.

444. Kamar N, Mariat C, Delahousse M, Dantal J, Al Najjar A, Cassuto E, et al. Diabetes mellitus after kidney transplantation: a French multicentre observational study. Nephrol Dial Transplant 2007;22:19861993.

445. Fabrizi F, Martin P, Dixit V, Bunnapradist S, Kanwal F, Dulai G. Posttransplant diabetes mellitus and HCV seropositive status after renal transplantation: meta-analysis of clinical studies. Am J Transplant 2005;5:2433-2440.

446. Roth D, Cirocco R, Zucker K, Ruiz P, Viciana A, Burke G, et al. De novo membranoproliferative glomerulonephritis in hepatitis $C$ virus- infected renal allograft recipients. Transplantation 1995;59:16761682.

447. Choy BY, Chan TM, Lai KN. Recurrent glomerulonephritis after kidney transplantation. Am J Transplant 2006;6:2535-2542.

448. Pockros PJ, Reddy KR, Mantry PS, Cohen E, Bennett M, Sulkowski MS, et al. L01: Safety of ombitasvir/paritaprevir/ritonavir plus dasabuvir for treating HCV GT1 infection in patients with severe renal impairment or end-stage renal disease: The RUBY-I study. [Abstract]. J Hepatol 2015;62(Suppl 2):S257.

449. Bruchfeld A, Lindahl K, Reichard O, Carlsson T, Schvarcz R. Pegylated interferon and ribavirin treatment for hepatitis $C$ in haemodialysis patients. J Viral Hepat 2006;13:316-321.

450. Garini G, Allegri L, Carnevali L, Catellani W, Manganelli P, Buzio C. Interferon-alpha in combination with ribavirin as initial treatment for hepatitis C virus-associated cryoglobulinemic membranoproliferative glomerulonephritis. Am J Kidney Dis 2001;38:E35.

451. Mazzaro C, Zorat F, Caizzi M, Donada C, Di Gennaro G, Maso LD, et al. Treatment with peg-interferon alfa-2b and ribavirin of hepatitis C virus-associated mixed cryoglobulinemia: a pilot study. J Hepatol 2005;42:632-638

452. Saadoun D, Resche-Rigon M, Thibault V, Piette JC, Cacoub P. Antiviral therapy for hepatitis $C$ virus--associated mixed cryoglobulinemia vasculitis: a long-term followup study. Arthritis Rheum 2006:54:3696-3706.

453. Lee SH, Kim KH, Lee SG, Cho H, Chen DH, Chung JS, et al. Causes of death and risk factors for mortality among HIV-infected patients receiving antiretroviral therapy in Korea. J Korean Med Sci 2013;28:990-997.

454. Bonacini M, Lin HJ, Hollinger FB. Effect of coexisting HIV-1 infection on the diagnosis and evaluation of hepatitis C virus. J Acquir Immune Defic Syndr 2001;26:340-344.

455. Marcellin $P$, Martinot-Peignoux M, Elias A, Branger M, Courtois $F$, Level $R$, et al. Hepatitis $C$ virus (HCV) viremia in human immunodeficiency virus-seronegative and -seropositive patients with indeterminate HCV recombinant immunoblot assay. J Infect Dis 1994;170:433-435.

456. Thomas DL, Astemborski J, Rai RM, Anania FA, Schaeffer M, Galai $N$, et al. The natural history of hepatitis $C$ virus infection: host, viral, and environmental factors. JAMA 2000;284:450-456.

457. Goedert JJ, Eyster ME, Lederman MM, Mandalaki T, De Moerloose P, White GC 2nd, et al. End-stage liver disease in persons with hemophilia and transfusion-associated infections. Blood 2002;100:15841589.

458. Thomas DL, Shih JW, Alter HJ, Vlahov D, Cohn S, Hoover DR, et al. Effect of human immunodeficiency virus on hepatitis $C$ virus infection among injecting drug users. J Infect Dis 1996;174:690-695.

459. Torriani FJ, Rodriguez-Torres M, Rockstroh JK, Lissen E, GonzalezGarcía J, Lazzarin A, et al. Peginterferon Alfa-2a plus ribavirin for 
The Korean Association for the Study of the Liver (KASL) KASL clinical practice guidelines: Management of Hepatitis C

chronic hepatitis C virus infection in HIV-infected patients. N Engl J Med 2004;351:438-450.

460. Bica I, McGovern B, Dhar R, Stone D, McGowan K, Scheib R, et al. Increasing mortality due to end-stage liver disease in patients with human immunodeficiency virus infection. Clin Infect Dis 2001;32:492-497.

461. Palella FJ Jr, Delaney KM, Moorman AC, Loveless MO, Fuhrer J, Satten $\mathrm{GA}$, et al. Declining morbidity and mortality among patients with advanced human immunodeficiency virus infection. HIV Outpatient Study Investigators. N Engl J Med 1998;338:853-860.

462. Weber R, Sabin CA, Friis-Møller N, Reiss P, El-Sadr WM, Kirk 0 , et al. Liver-related deaths in persons infected with the human immunodeficiency virus: the D:A:D study. Arch Intern Med 2006;166:1632-1641.

463. Qurishi N, Kreuzberg C, Lüchters G, Effenberger W, Kupfer B, Sauerbruch $\mathrm{T}$, et al. Effect of antiretroviral therapy on liver-related mortality in patients with HIV and hepatitis C virus coinfection. Lancet 2003;362:1708-1713.

464. Tien PC. Veterans Affairs Hepatitis C Resource Center Program; National Hepatitis C Program Office. Management and treatment of hepatitis $\mathrm{C}$ virus infection in HIV-infected adults: recommendations from the Veterans Affairs Hepatitis C Resource Center Program and National Hepatitis C Program Office. Am J Gastroenterol 2005;100:2338-2354.

465. Avidan NU, Goldstein D, Rozenberg L, McLaughlin M, Ferenci P, Masur $\mathrm{H}$, et al. Hepatitis $\mathrm{C}$ viral kinetics during treatment with peg IFN-alpha-2b in HIV/HCV coinfected patients as a function of baseline CD4 $₫$ T-cell counts. J Acquir Immune Defic Syndr 2009;52:452458.

466. Sulkowski MS, Mehta SH, Torbenson MS, Higgins Y, Brinkley SC, de Oca RM, et al. Rapid fibrosis progression among HIV/hepatitis C virus-co-infected adults. AIDS 2007;21:2209-2216.

467. Bräu N, Salvatore M, Ríos-Bedoya CF, Fernández-Carbia A, Paronetto F, Rodríguez-Orengo JF, et al. Slower fibrosis progression in HIV/HCV-coinfected patients with successful HIV suppression using antiretroviral therapy. J Hepatol 2006;44:47-55.

468. Macías J, Berenguer J, Japón MA, Girón JA, Rivero A, López-Cortés $L F$, et al. Fast fibrosis progression between repeated liver biopsies in patients coinfected with human immunodeficiency virus/hepatitis C virus. Hepatology 2009;50:1056-1063.

469. Opravil M, Sasadeusz J, Cooper DA, Rockstroh JK, Clumeck N, Clotet $B$, et al. Effect of baseline CD4 cell count on the efficacy and safety of PegIFN-a Alfa-2a (40KD) plus ribavirin in patients with HIV/hepatitis C virus coinfection. J Acquir Immune Defic Syndr 2008;47:36-49.

470. Sulkowski MS. Management of acute and chronic HCV infection in persons with HIV coinfection. J Hepatol 2014;61(1 Suppl):S108S119.
471. Alvarez D, Dieterich DT, Brau N, Moorehead L, Ball L, Sulkowski MS. Zidovudine use but not weight-based ribavirin dosing impacts anaemia during HCV treatment in HIV-infected persons. J Viral Hepat 2006;13:683-689.

472. Hoggard PG, Kewn S, Barry MG, Khoo SH, Back DJ. Effects of drugs on 2',3'-dideoxy- 2', 3'-didehydrothymidine phosphorylation in vitro. Antimicrob Agents Chemother 1997;41:1231-1236.

473. Lafeuillade A, Hittinger G, Chadapaud S. Increased mitochondrial toxicity with ribavirin in HIV/HCV coinfection. Lancet 2001;357:280-281.

474. Salmon-Céron D, Chauvelot-Moachon L, Abad S, Silbermann B, Sogni P. Mitochondrial toxic effects and ribavirin. Lancet 2001;357:1803-1804.

475. Osinusi A, Townsend K, Kohli A, Nelson A, Seamon C, Meissner $E G$, et al. Virologic response following combined ledipasvir and sofosbuvir administration in patients with HCV genotype 1 and HIV co-infection. JAMA 2015;313:1232-1239.

476. Sulkowski MS, Eron JJ, Wyles D, Trinh R, Lalezari J, Wang C, et al. Ombitasvir, paritaprevir co-dosed with ritonavir, dasabuvir, and ribavirin for hepatitis $C$ in patients co-infected with HIV-1: a randomized trial. JAMA 2015;313:1223-1231.

477. Naggie S, Cooper C, Saag M, Workowski K, Ruane P, Towner WJ, et al. Ledipasvir and Sofosbuvir for HCV in patients coinfected with HIV-1. N Engl J Med 2015;373:705-713.

478. Sulkowski MS, Naggie S, Lalezari J, Fessel WJ, Mounzer K, Shuhart $M$, et al. Sofosbuvir and ribavirin for hepatitis $C$ in patients with HIV coinfection. JAMA2014;312:353-361.

479. Núñez M, Miralles C, Berdún MA, Losada E, Aguirrebengoa K, Ocampo $A$, et al. Role of weight-based ribavirin dosing and extended duration of therapy in chronic hepatitis C in HIV-infected patients: the PRESCO trial. AIDS Res Hum Retroviruses 2007;23:972-982.

480. Ghany MG, Strader DB, Thomas DL, Seeff LB. American Association for the Study of Liver Diseases. Diagnosis, management, and treatment of hepatitis C: an update. Hepatology 2009;49:13351374.

481. Cargnel A, Angeli E, Mainini A, Gubertini G, Giorgi R, Schiavini M, et al. Open, randomized, multicentre italian trial on PEG-IFN plus ribavirin versus PEG-IFN monotherapy for chronic hepatitis $C$ in HIV-coinfected patients on HAART. Antivir Ther 2005;10:309-317.

482. Santin M, Shaw E, Garcia MJ, Delejido A, de Castro ER, Rota R, et al. Efficacy and safety of pegylated interferon-alpha2b plus ribavirin for the treatment of chronic hepatitis C in HIV-infected patients. AIDS Res Hum Retroviruses 2006;22:315-320.

483. Núnez $M$, Camino N, Ramos $B$, Berdún MA, Barreiro $P$, Losada $E$, et al. Impact of ribavirin exposure on early virological response to hepatitis $\mathrm{C}$ therapy in HIV-infected patients with chronic hepatitis $\mathrm{C}$. Antivir Ther 2005;10:657-662.

484. Fernández-Montero JV, Soriano V. Management of hepatitis C in 
HIV and/or HBV co-infected patients. Best Pract Res Clin Gastroenterol 2012;26:517-530.

485. Kim YJ, Lee JW, Kim YS, Jeong SH, Kim YS, Yim HJ, et al. Clinical features and treatment efficacy of PegIFN-a alfa plus ribavirin in chronic hepatitis C patients coinfected with hepatitis B virus. Korean J Hepatol 2011;17:199-205.

486. Chiaramonte M, Stroffolini T, Vian A, Stazi MA, Floreani A, Lorenzoni $U$, et al. Rate of incidence of hepatocellular carcinoma in patients with compensated viral cirrhosis. Cancer 1999;85:21322137.

487. Lee LP, Dai CY, Chuang WL, Chang WY, Hou NJ, Hsieh MY, et al. Comparison of liver histopathology between chronic hepatitis $C$ patients and chronic hepatitis B and C-coinfected patients. J Gastroenterol Hepatol 2007;22:515-517.

488. Sagnelli E, Pasquale G, Coppola N, Scarano F, Marrocco C, Scolastico $C$, et al. Influence of chronic coinfection with hepatitis $B$ and $C$ virus on liver histology. Infection 2004;32:144-148.

489. Benvegnù L, Fattovich G, Noventa F, Tremolada F, Chemello L, Cecchetto $A$, et al. Concurrent hepatitis $B$ and $C$ virus infection and risk of hepatocellular carcinoma in cirrhosis. A prospective study. Cancer 1994;74:2442-2448.

490. Potthoff A, Manns MP, Wedemeyer H. Treatment of HBV/HCV coinfection. Expert Opin Pharmacother 2010;11:919-928.

491. Potthoff A, Wedemeyer H, Boecher WO, Berg T, Zeuzem S, Arnold J, et al. The HEP-NET B/C co-infection trial: a prospective multicenter study to investigate the efficacy of pegylated interferon-alpha2b and ribavirin in patients with HBV/HCV co-infection. J Hepatol 2008;49:688-694.

492. Liu CJ, Chuang WL, Lee CM, Yu ML, Lu SN, Wu SS, et al. Peginterferon alfa-2a plus ribavirin for the treatment of dual chronic infection with hepatitis B and C viruses. Gastroenterology 2009;136:496-504.e3.

493. Collins JM, Raphael KL, Terry C, Cartwright EJ, Pillai A, Anania FA, et al. Hepatitis $B$ virus reactivation during successful treatment of Hepatitis C virus with Sofosbuvir and Simeprevir. Clin Infect Dis 2015;61:1304-1306.

494. Potthoff A, Berg T, Wedemeyer H. HEP-NET B/C Coinfection Study Group. Late hepatitis B virus relapse in patients co-infected with hepatitis B virus and hepatitis C virus after antiviral treatment with pegylated interferon-a2b and ribavirin. Scand J Gastroenterol 2009;44:1487-1490.

495. Asian Pacific Association for the Study of the Liver (APASL) Hepatitis C Working Party, McCaughan GW, Omata M, Amarapurkar D, Bowden S, Chow WC, et al. Asian Pacific Association for the Study of the Liver consensus statements on the diagnosis, management and treatment of hepatitis C virus infection. J Gastroenterol Hepatol 2007;22:615-633.

496. Vento S, Cainelli F, Cesario F. Infections and thalassaemia. Lancet
Infect Dis 2006;6:226-233.

497. Plug I, Van Der Bom JG, Peters M, Mauser-Bunschoten EP, De Goede-Bolder A, Heijnen L, et al. Mortality and causes of death in patients with hemophilia, 1992-2001: a prospective cohort study. J Thromb Haemost 2006;4:510-516.

498. Zhang M, Rosenberg PS, Brown DL, Preiss L, Konkle BA, Eyster $M E$, et al. Correlates of spontaneous clearance of hepatitis $C$ virus among people with hemophilia. Blood 2006;107:892-897.

499. Maor Y, Bashari D, Kenet G, Lubetsky A, Luboshitz J, Schapiro $J M$, et al. Non-invasive biomarkers of liver fibrosis in haemophilia patients with hepatitis C: can you avoid liver biopsy? Haemophilia 2006;12:372-379.

500. Honda T, Katano Y, Kuzuya T, Hayashi K, Ishigami M, Itoh A, et al. Comparison of the efficacy of ribavirin plus PeglFN-a alfa-2b for chronic hepatitis $C$ infection in patients with and without coagulation disorders. J Med Virol 2013;85:228-234.

501. Yang SY, Lee HW, Lee YJ, Park SJ, Yoo KY, Kim HJ. Highly effective PegIFN-a $\alpha$-2a plus ribavirin combination therapy for chronic hepatitis $C$ in hemophilia in Korea. Clin Mol Hepatol 2015;21:125-130.

502. Ozguroglu M, Bilici A, Turna $H$, Serdengecti S. Reactivation of hepatitis $B$ virus infection with cytotoxic therapy in non-Hodgkin's lymphoma. Med Oncol 2004;21:67-72.

503. Yeo W, Chan PK, Zhong S, Ho WM, Steinberg JL, Tam JS, et al. Frequency of hepatitis $B$ virus reactivation in cancer patients undergoing cytotoxic chemotherapy: a prospective study of 626 patients with identification of risk factors. J Med Virol 2000;62:299-307.

504. Kawatani T, Suou T, Tajima F, Ishiga K, Omura H, Endo A, et al. Incidence of hepatitis virus infection and severe liver dysfunction in patients receiving chemotherapy for hematologic malignancies. Eur J Haematol 2001;67:45-50.

505. Markovic S, Drozina G, Vovk M, Fidler-Jenko M. Reactivation of hepatitis $B$ but not hepatitis $C$ in patients with malignant lymphoma and immunosuppressive therapy. A prospective study in 305 patients. Hepatogastroenterology 1999;46:2925-2930.

506. Vento S, Cainelli F, Longhi MS. Reactivation of replication of hepatitis $B$ and $C$ viruses after immunosuppressive therapy: an unresolved issue. Lancet Oncol 2002;3:333-340.

507. Faggioli P, De Paschale M, Tocci A, Luoni M, Fava S, De Paoli A, et al. Acute hepatic toxicity during cyclic chemotherapy in non Hodgkin's lymphoma. Haematologica 1997;82:38-42.

508. Nosotti L, D’Andrea M, Pitidis A, Pimpinelli F, Dessanti ML, Pisani F, et al. Hepatitis $C$ virus infection prevalence and liver dysfunction in a cohort of B-cell non-Hodgkin's lymphoma patients treated with immunochemotherapy. Scand J Infect Dis 2012;44:70-73.

509. Takai S, Tsurumi H, Ando K, Kasahara S, Sawada M, Yamada T, et al. Prevalence of hepatitis $B$ and $C$ virus infection in haematological malignancies and liver injury following chemotherapy. Eur J Haematol 2005;74:158-165. 
The Korean Association for the Study of the Liver (KASL) KASL clinical practice guidelines: Management of Hepatitis C

510. de Pree C, Giostra E, Galetto A, Perrin L, Zulian GB. Hepatitis C virus acute exacerbation during chemotherapy and radiotherapy for oesophageal carcinoma. Ann Oncol 1994;5:861-862.

511. Melisko ME, Fox R, Venook A. Reactivation of hepatitis $C$ virus after chemotherapy for colon cancer. Clin Oncol (R Coll Radiol) 2004;16:204-205

512. Fan FS, Tzeng CH, Hsiao KI, Hu ST, Liu WT, Chen PM. Withdrawal of immunosuppressive therapy in allogeneic bone marrow transplantation reactivates chronic viral hepatitis C. Bone Marrow Transplant 1991;8:417-420.

513. Kanamori H, Fukawa H, Maruta A, Harano H, Kodama F, Matsuzaki $M$, et al. Case report: fulminant hepatitis C viral infection after allogeneic bone marrow transplantation. Am J Med Sci 1992;303:109111.

514. Vento S, Cainelli F, Mirandola F, Cosco L, Di Perri G, Solbiati M, et al. Fulminant hepatitis on withdrawal of chemotherapy in carriers of hepatitis C virus. Lancet 1996;347:92-93.

515. Nakamura Y, Motokura T, Fujita A, Yamashita T, Ogata E. Severe hepatitis related to chemotherapy in hepatitis $B$ virus carriers with hematologic malignancies. Survey in Japan, 1987-1991. Cancer 1996;78:2210-2215.

516. Locasciulli A, Bruno B, Alessandrino EP, Meloni G, Arcese W, Bandini $G$, et al. Hepatitis reactivation and liver failure in haemopoietic stem cell transplants for hepatitis B virus (HBV)/hepatitis C virus ( $\mathrm{HCV}$ ) positive recipients: a retrospective study by the Italian group for blood and marrow transplantation. Bone Marrow Transplant 2003;31:295-300.

517. Hamaguchi M, Yamada H, Gondo H, Takemoto Y, Morishima Y, Kodera $Y$. Retrospective study on the impact of hepatitis $B$ and hepatitis $C$ virus infection on hematopoietic stem cell transplantation in Japan. Int J Hematol 2002;75:324-331.

518. Lee JM, Lee JM, Yoo HS, Jang UK, Kim DJ, Kim YB, et al. The prevalence of anti-HCV positivity in healthy Korean children. Korean J Hepatol 1996;2:160-165.

519. Mohan P, Barton BA, Narkewicz MR, Molleston JP, Gonzalez-Peralta RP, Rosenthal $\mathrm{P}$, et al. Evaluating progression of liver disease from repeat liver biopsies in children with chronic hepatitis C: a retrospective study. Hepatology 2013;58:1580-1586.

520. Pembrey L, Newell ML, Tovo PA. EPHN Collaborators. The management of HCV infected pregnant women and their children European paediatric HCV network. J Hepatol 2005;43:515-525.

521. Airoldi J, Berghella V. Hepatitis C and pregnancy. Obstet Gynecol
Surv 2006:61:666-672.

522. Hepatitis $C$ virus infection. American Academy of Pediatrics. Committee on Infectious Diseases. Pediatrics 1998;101(3 Pt 1):481-485.

523. Mast EE, Hwang LY, Seto DS, Nolte FS, Nainan OV, Wurtzel $H$, et al. Risk factors for perinatal transmission of hepatitis C virus (HCV) and the natural history of HCV infection acquired in infancy. J Infect Dis 2005;192:1880-1889.

524. Palomba E, Manzini P, Fiammengo P, Maderni P, Saracco G, Tovo PA. Natural history of perinatal hepatitis $C$ virus infection. Clin Infect Dis 1996;23:47-50.

525. Polywka S, Pembrey L, Tovo PA, Newell ML. Accuracy of HCV-RNA $P C R$ tests for diagnosis or exclusion of vertically acquired HCV infection. J Med Virol 2006;78:305-310.

526. Guido M, Rugge M, Jara P, Hierro L, Giacchino R, Larrauri J, et al. Chronic hepatitis $C$ in children: the pathological and clinical spectrum. Gastroenterology 1998;115:1525-1529.

527. Mack CL, Gonzalez-Peralta RP, Gupta N, Leung D, Narkewicz MR, Roberts EA, et al. NASPGHAN practice guidelines: diagnosis and management of hepatitis $C$ infection in infants, children, and adolescents. J Pediatr Gastroenterol Nutr 2012;54:838-855.

528. El Sherbini A, Mostafa S, Ali E. Systematic review with metaanalysis: comparison between therapeutic regimens for paediatric chronic hepatitis C. Aliment Pharmacol Ther 2015;42:12-19.

529. González-Peralta RP, Kelly DA, Haber B, Molleston J, Murray KF, Jonas $\mathrm{MM}$, et al. Interferon alfa-2b in combination with ribavirin for the treatment of chronic hepatitis C in children: efficacy, safety, and pharmacokinetics. Hepatology 2005;42:1010-1018.

530. Wirth S, Lang T, Gehring S, Gerner P. Recombinant alfa-interferon plus ribavirin therapy in children and adolescents with chronic hepatitis C. Hepatology 2002;36:1280-1284.

531. Wirth S, Pieper-Boustani H, Lang T, Ballauff A, Kullmer U, Gerner P, et al. Peginterferon alfa-2b plus ribavirin treatment in children and adolescents with chronic hepatitis C. Hepatology 2005;41:10131018.

532. Wirth S, Ribes-Koninckx C, Calzado MA, Bortolotti F, Zancan L, Jara $\mathrm{P}$, et al. High sustained virologic response rates in children with chronic hepatitis C receiving PeglFN-a alfa-2b plus ribavirin. J Hepatol 2010;52:501-507.

533. Schwarz KB, Gonzalez-Peralta RP, Murray KF, Molleston JP, Haber $\mathrm{BA}$, Jonas MM, et al. The combination of ribavirin and PegIFN-a is superior to PegIFN-a and placebo for children and adolescents with chronic hepatitis C. Gastroenterology 2011;140:450-458.e1. 